\title{
Superconductor-to-normal transitions in dissipative chains of mesoscopic grains and nanowires
}

\section{Citation}

Refael, Gil, Eugene Demler, Yuval Oreg, and Daniel S. Fisher. 2007. “Superconductor-to-Normal Transitions in Dissipative Chains of Mesoscopic Grains and Nanowires." Physical Review B 75 (1) (January 18). doi:10.1103/physrevb.75.014522.

\section{Published Version}

doi:10.1103/PhysRevB.75.014522

\section{Permanent link}

http://nrs.harvard.edu/urn-3:HUL.InstRepos:27891923

\section{Terms of Use}

This article was downloaded from Harvard University's DASH repository, and is made available under the terms and conditions applicable to Other Posted Material, as set forth at http:// nrs.harvard.edu/urn-3:HUL.InstRepos:dash.current.terms-of-use\#LAA

\section{Share Your Story}

The Harvard community has made this article openly available.

Please share how this access benefits you. Submit a story.

\section{Accessibility}




\title{
Superconductor-to-normal transitions in dissipative chains of mesoscopic grains and nanowires
}

\author{
Gil Refael, ${ }^{1}$ Eugene Demler, ${ }^{2}$ Yuval Oreg, ${ }^{3}$ and Daniel S. Fisher ${ }^{2}$ \\ ${ }^{1}$ Department of Physics, California Institute of Technology, Pasadena, California 91125, USA \\ ${ }^{2}$ Department of Physics, Harvard University, Cambridge, Massachusetts 02138, USA \\ ${ }^{3}$ Department of Condensed Matter Physics, Weizmann Institute of Science, Rehovot, 76100, Israel \\ (Received 27 December 2005; revised manuscript received 10 November 2006; published 18 January 2007)
}

\begin{abstract}
The interplay of quantum fluctuations and dissipation in chains of mesoscopic superconducting grains is analyzed and the results are applied to nanowires. It is shown that in one dimensional arrays of resistively shunted Josephson junctions, the superconducting-normal charge relaxation within the grains plays an important role. At zero temperature, two superconducting phases can exist, depending primarily on the strength of the dissipation. In the fully superconducting phase (FSC), each grain acts superconducting, and the coupling to the dissipative conduction is important. In the $\mathrm{SC}^{\star}$ phase, the dissipation is irrelevant at long wavelengths. The transition between these two phases is driven by quantum phase slip dipoles, and is primarily local, with continuously varying critical exponents. In contrast, the transition from the $\mathrm{SC}^{\star}$ phase to the normal metallic phase is a Kosterlitz-Thouless transition with global character (i.e., determined by the field behavior at large wavelengths). Most interesting is the transition from the FSC phase directly to the normal phase: this transition, which has mixed local and global characteristics, can be one of three distinct types. The corresponding segments of the phase boundary come together at bicritical points. The zero-temperature phase diagram, as well as the finite-temperature scaling behavior are inferred from both weak and strong coupling renormalization group analyses. At intermediate temperatures, near either superconductor-to-normal phase transition, there are regimes of super-metallic behavior, in which the resistivity first decreases gradually with decreasing temperature before eventually increasing as temperature is lowered further. The results on chains of Josephson junctions are extended to continuous superconducting nanowires and the subtle issue of whether these can exhibit an FSC phase is considered. Potential relevance to superconductor-metal transitions in other systems is also discussed.
\end{abstract}

DOI: 10.1103/PhysRevB.75.014522

PACS number(s): 74.81.Fa, 74.78.Na

\section{INTRODUCTION}

\section{A. Motivation}

Quantum mechanical systems that are coupled to dissipative "environments" arise in many areas of physics, including spin dynamics in nuclear magnetic resonance, ${ }^{1,2}$ damping in atomic clocks and optical interferometers, ${ }^{3}$ dephasing and decoherence in mesoscopic systems, ${ }^{4}$ and quantum computing. ${ }^{5,6}$ Also in more conventional condensed matter contexts, dissipation has been argued to play crucial roles. In particular: near to quantum magnetic phase transitions, ${ }^{7-9}$ in quantum Hall systems, ${ }^{10}$ and in various aspects of superconductivity, including Josephson junctions and thin superconducting films and wires. ${ }^{11-24}$ The last of these is the primary focus of the present paper.

For theoretical studies of dissipative effects in macroscopic_and some mesoscopic_quantum systems, the degrees of freedom that cause the dissipation are often modeled as a heat bath following Caldeira and Leggett. ${ }^{25-27}$ The best studied example is a single resistively-shunted Josephson junction (RSJJ). ${ }^{28-34}$ Recent experiments by Pentillä et al. ${ }^{35,36}$ have shown good agreement with the theoretical predictions. Extensions to arrays of RSJJs have been analyzed by several groups focusing on the existence and location of phase boundaries between superconducting and insulating regimes of the set of junctions. ${ }^{11,37-47}$ Experimental studies of one and two dimensional arrays of large superconducting grains coupled by dissipative Josephson junctions, agree qualitatively with results of the theoretical analyses. ${ }^{46,48-58}$
The understanding developed from studying the destruction of superconductivity by quantum fluctuations in arrays of Josephson junctions has became a useful paradigm for more general considerations of quantum phase transitions in dissipative environments. In general, dissipation suppresses certain types of quantum fluctuations and thus can favor states with spontaneously broken symmetries, such as superconductivity. Considerable interest in further theoretical analysis of RSJJ arrays thus stems not only from direct experimental relevance in the context of superconductivity, but also from expectations that the concepts and approaches will be useful far more generally, especially for understanding universal aspects of quantum phase transitions that can occur at zero temperature in the presence of dissipation. However, to do this, it is crucial to take into account the small size of the components involved, whether they are small grains or individual atoms in a crystal.

Because the models on which they are based were initially introduced to understand macroscopic quantum phenomena, ${ }^{25-27}$ most theoretical analyses of RSJJ arrays have assumed that the effective charges associated with the superconducting and normal currents are perfectly mixed within the superconducting grains while passing separately between grains via the Josephson junctions and normal "shunts," respectively. Such an approximation is reasonable for macroscopic superconductors but will break down in mesoscopic or microscopic systems. ${ }^{59}$ As we showed in Ref. 59, a consequence of the breakdown of the macroscopic paradigm is that the superconducting and normal fluids can effectively decouple at low energies. For the simple case of 
two junctions in series through a mesoscopic grain, we showed that this leads to changes in both the nature and location of the superconductor-normal transition that occurs. In the present paper, this analysis is extended to show that decoupling of the two fluids has equally important consequences for chains of mesoscopic grains and for superconducting nanowires.

The goal of this paper is to provide a detailed analysis of superconductor-to-normal transitions in one dimensional mesoscopic systems for which dissipation plays a role. The main emphasis is on chains of mesoscopic grains that are connected both by Josephson junctions and some form of shunt resistance, although we also consider continuous superconducting nanowires. We will not discuss the possible origins of the assumed Ohmic dissipation in such systems, but rather assume that it is present and study its consequences. Furthermore, because we are primarily interested in dissipative effects that arise simply only if the diameters of the grains or wires are substantially larger than atomic sizes, we will also ignore the limits in which the discreteness of the electrons or Cooper pairs becomes most essential, such as in Giamarchi and Schulz's treatment of superfluid-to-normal transitions in Luttinger liquids, ${ }^{60}$ and phenomena associated with localization by randomness. Within a simple but relatively general mesoscopic model, we analyze the nature of the phases that can exist and the locations and character of the several types of quantum phase transitions that occur. In particular, we study the universal scaling behavior of the resistivity in the vicinity of the superconductor-to-normal transition(s). To do so, we develop both strong- and weakcoupling renormalization group approaches which are tailored to deal with both the local resistive and the longwavelength superconducting degrees of freedom.

\section{B. Outline}

This paper is organized as follows:

Section I provides a general introduction. Section I C introduces the two-fluid approach to mesoscopic superconducting grains, as first given in Ref. 59. Section ID gives a summary of the main results of the paper, omitting technical details.

Section II derives the quantum two fluid model that describes an infinite chain of mesoscopic two-fluid grains as shown in Fig. 1. Section II A discusses the various possible regimes and Sec. II B gives an analysis of the linear electrodynamics of the model which provides intuition for the location and nature of the various transitions.

In Sec. III we discuss the strong coupling limit of the chain, first deriving the quantum phase-slip representation of the chain (Sec. III A), from which we construct a sineGordon action (Sec. III B). Using the sine-Gordon action for the chain, we discuss the possible phases of the system (Sec. III C). Finally, in Sec. III D we derive the strong coupling renormalization-group ( $\mathrm{RG}$ ) flow equations for the system. These are constructed by an anisotropic scaling procedure, suited to the dissipative environment.

In Sec. IV we use the RG flow equations to determine the phase diagram of the system. Although the system exhibits (a) Superconducting mesoscopic grains

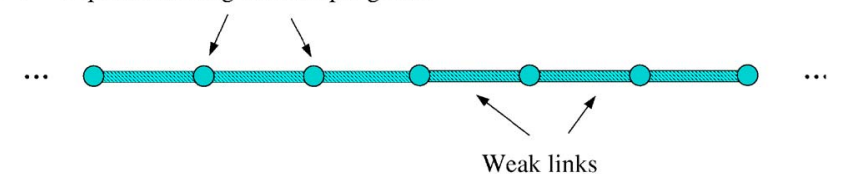

(b)

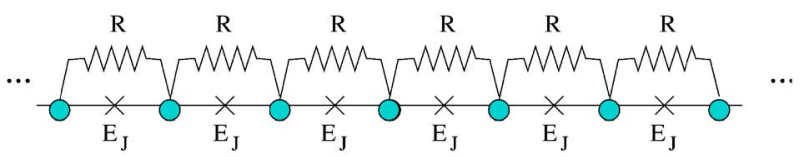

(c)

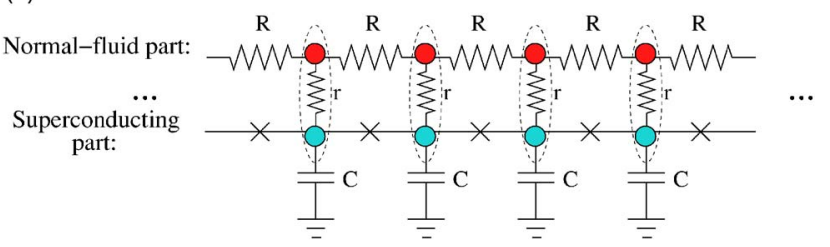

FIG. 1. (Color online) (a) A system of superconducting grains, which we assume have gapless excitations due to disorder and dynamical effects, connected by weak links. The grains are coupled by flows of both superconducting Cooper pairs and normal electrons. (b) We model the system in (a) by a chain of superconducting grains connected by Josephson junctions and shunt resistors. (c) Two fluid model: each mesoscopic grain is represented as a combination of a superconducting and a normal parts, depicted here as separate grains. Charge relaxation between the $S$ and $N$ grains is via a conversion resistance $r$.

three phases, the transitions between them have a variety of types. Each transition is discussed separately in Secs. IV A-IV F. Special aspects of the phase diagram, such as bicritical and multicritical points are discussed in Secs. IV G-IV I.

Section V analyzes the weak-Josephson coupling limit. First, we cast the action in terms of pair-tunnel events (Sec. $\mathrm{V} \mathrm{A}$ ). Then we construct the RG flow equations (Sec. V B), from which we obtain the weak coupling flow diagram (Sec. V C).

Section VI considers superconducting nanowires by considering them as the continuum limit of the JJ chains.

Section VII presents scaling forms for the resistivity of the chain and discusses various interesting parameter regimes.

We conclude in Sec. VIII by reviewing the implications of our results for various experimental systems and raising open questions.

Some technical details are relegated to the Appendix.

\section{Quantum two fluid description}

\section{Mesoscopic grains and shunted Josephson junctions}

The primary system we will study is shown in Fig. 1(a): a chain of identical mesoscopic superconducting grains which are connected by weak links that allow the flow of both Cooper pairs and normal electrons. Following Refs. 11, 39, and 40 we describe this system as a chain of resistively shunted Josephson junctions [Fig. 1(b)]. Such an RSJJ chain has a natural interpretation in terms of a "two fluid" model: 
Cooper pairs that can tunnel between the superconducting grains comprise the superfluid, and electrons that can flow in the shunting resistors the normal fluid. The presence of both fluids suggests considering each grain as consisting of two (physically overlapping) parts-a superconducting grain $(S)$ and a normal grain $(N)$-as shown in Fig. 1(c).

Changes in the super or normal charge on a grain will induce changes in the corresponding electrochemical potentials with the coefficients depending on both the capacitance of the grain and the compressibilities of the normal and superfluid components. A difference between the normal and the superfluid electrochemical potentials will lead to charge relaxation between them; we model this as a conversion current $I_{N S}$ through a phenomenological Ohmic conversion resistance $r$.

This classical model of a chain of grains can be made into a quantum model straightforwardly by analogy with previous work, e.g., Ref. 59. The length scale is set by the spacing $a$ between the grain centers, and various time scales by the normal-superfluid relaxation rate within a grain, the plasma frequency of the Josephson junctions, and the high frequency cutoffs of the superconducting degrees of freedom (typically of order the energy gap) and of the dissipative processes.

We will show that the behavior of the quantum chain depends crucially on the normal-superfluid conversion resistance $r$. In the limit $r \rightarrow 0$ the relaxation between the $S$ and $N$ fluids is infinitely fast, and our model reduces to that of an RSJJ chain composed of macroscopic superconducting grains each with a single electrochemical potential.

This is the limit studied previously. ${ }^{41-43,45}$ The opposite limit $r \rightarrow \infty$ describes a system with purely capacitative couplings between the $S$ and the $N$ fluids. The analog of this case in two dimensions has been discussed in Refs. 61 and 62 and also realized experimentally. ${ }^{53,63}$ The intermediate case of finite $r$ involves new behavior, which, to our knowledge, has not been analyzed previously: it should be relevant for experiments on arrays of mesoscopic grains. The new physics associated with the interplay between the $S$ and $N$ fluids dominates below a temperature $T^{*}$, roughly proportional to the electron-energy-level spacing within a grain. ${ }^{59}$

\section{Nanowires}

The two fluid model can be readily generalized to a continuous wire that is thin enough to ignore dependencies on the transverse coordinates. Classically, the corresponding two-fluid model is defined by the generalized Josephson equation for the superfluid current, Ohm's law for the normal current, and a constitutive equation for the conversion current

$$
\begin{gathered}
\frac{\partial I_{S}}{\partial t}=-\Upsilon \nabla V_{S}, \\
I_{N}=-\sigma \nabla V_{N}, \\
\mathcal{I}_{N S}=\gamma\left(V_{N}-V_{S}\right)
\end{gathered}
$$

with $V_{S}$ and $V_{N}$ the electrochemical potentials for the superfluid and normal electrons, respectively. Note that for a wire, the conversion current $\mathcal{I}_{\mathrm{NS}}$ is a current per unit length, so $\gamma$ has dimensions conductance per unit length. $\gamma$, the conductivity between the normal and superfluid parts, reflects the relaxation rate for a population imbalance between the two fluids, as investigated, e.g., by Clarke et al. ${ }^{64}$ The current equations must be supplemented, as for the chain of grains, by current conservation laws and constitutive relations between the excess normal and superfluid charge densities and the electrochemical potentials; again these will involve compressibilities and capacitances.

The continuum two fluid equations (1) can be made into a quantum model by defining the superfluid velocity as the gradient of the superconducting phase, imposing phasecharge conjugation, and introducing appropriate degrees of freedom to mimic the dissipation associated with the resistive processes. In addition, the superconducting phase should be allowed to undergo quantum phase slips (QPS); these are implicit in the chain of grains but not included in the linear continuum model (for a discussion of phase slips see Sec. III). To introduce phase slips, a short distance cutoff must be imposed as, in contrast to the chain, there is no intrinsic length scale. Care must be exercised, however, when imposing a cutoff on this nanowire model. For example, a simple lattice regularization implicitly assumes that the size of quantum phase slips is also the shortest possible distance between them. In reality the core size of a QPS is nonzero but, if two phase slips occur at different times, their centers can be arbitrarily close to one other. We discuss this important subtlety in detail in Sec. VI.

\section{Overview of results}

Before formulating the quantum model of a chain of grains and analyzing it in detail, we give a brief overview of the results obtained in this paper. The main elements developed in this paper are twofold. First, the effects due to a finite $N$ to $S$ relaxation resistance. Second, our analysis is based on the RG approach to this problem which we have developed, and which is applied both in the strong and weak coupling limits. This development of the RG approach allows us not only to obtain the phase diagram, which in the limit $r=0$ was by and large obtained before, ${ }^{41-43,45}$ but also understand the intricate crossovers in the system, and obtain scaling forms for its transport properties. Wherever possible we will note the overlap and difference with previously obtained results.

\section{Phases}

The JJ chain has two superfluid phases, which we call fully superconducting (FSC) and $\mathrm{SC}^{\star}$, plus a normal metallic phase (NOR). [The two types of superconducting phases were discussed earlier in Refs. 41-43 and 45 and referred to as SC-2 and SC-1.] In the FSC phase, which occurs when dissipation is strong, quantum fluctuations are suppressed enough that conventional superconducting tunneling into a grain is possible, and the Josephson junctions behaves completely classically at zero temperature. Nevertheless the superconducting correlations are not truly long range, but decay algebraically with distance. In the opposite limit, the 
normal phase, phase fluctuations are large, and number fluctuations are small, so that the charge of a grain is well defined and superconducting correlations decay exponentially.

In between the FSC and NOR phases is the $\mathrm{SC}^{\star}$ phase, in which both the phases and the charges of individual grains exhibit large fluctuations. Phase differences between grains, however, do exhibit quasi-long-range order. In this remarkable phase, tunneling into a grain will result in singular low energy behavior, and will be suppressed, which is an indication of the strong fluctuation of the phase variables on each grain in this state. Nevertheless a uniform supercurrent can flow through the chain unimpeded at low energies (see also Refs. 41 and 59). The $\mathrm{SC}^{\star}$ phase tends to occur when the dissipation is weak: at low energies in the $\mathrm{SC}^{\star}$ phase, the dissipation is irrelevant with the quantitative values of the shunting $R$ and conversion $r$ resistances playing little role, in the determination of global transport properties.

The three phase structure is somewhat analogous to the behavior of two-dimensional solids describable by disclinations: at intermediate temperatures the disclinations bind into pairs forming dislocations-the hexatic phase with quasilong-range orientational order-and at lower temperatures, these dislocations bind into pairs-disclination quadrupoles-forming the solid phase which has true orientational long range order.

\section{Transitions}

Three types of quantum phase transitions occur between the three phases. We denote the critical values of parameters for these transitions $G, L$, and $M$ for global, local, and mixed, respectively.

Separating the $\mathrm{SC}^{\star}$ phase and the normal phase, is a transition of Kosterlitz-Thouless (KT) type driven by of unbinding pairs of quantum phase slips. It is thus controlled by an effective space-time phase "stiffness" parameter that we denote $K$, proportional to the square root of the ratio of the Josephson and capacitative energies: the former favors superconductivity, while the latter favors localization of charge, and hence normal behavior. Since the $\mathrm{SC}^{\star}$-NOR transition is intrinsically controlled by long-wavelength physics, we refer to it as global $(G)$. Interestingly, in this transition the resistive shunts play almost no role: they are screened by the fluctuations of phase slip dipoles, i.e., a pair of phase-slip and anti-phase-slip occurring simultaneously on neighboring junctions. The dynamical exponent $z$ that relates time or inverse-energy scales to length scales is, for this transition, equal to unity: $z_{G}=1$. Deviations from criticality are only marginally relevant, in the RG sense, and give rise to characteristic energy scales going to zero at the critical point with the exponential form typical of KT transitions.

Between the two superconducting phases, the transition is driven by dissipation. It is essentially local $(L)$, being related to the superconducting-normal transition of a single Josephson junction. The important excitations that control this local transition are phase slips between one grain and the rest of the chain. These excitations, in terms of individual quantum phase slips of the chain, are equivalent to quantum phase slip dipoles, which, as mentioned above, consist of a pair of opposite sign phase slips, one on each side of the grain. Asso- ciated with the locality of this physics, there is no simple diverging length scale and the dynamical exponent is thus $z_{L}=\infty$. How the characteristic energy scale goes to zero at this transition depends on values of resistances: the corresponding critical exponents vary continuously.

Perhaps the most interesting transition is that which can occur from the FSC phase directly to the normal phase: this is driven by changes in the dissipation, yet because it also involves destruction of superconductivity, it has mixed $(M)$ character with both local and global aspects and involves the interplay between individual quantum phase slips, and phase slip dipoles. Related to this more complicated mechanism, there is more than one type of critical behavior-probably three types-for the mixed transition.

Our understanding of the nature of the phase boundaries as described above draws on the discussions in Refs. 41 and 45. The RG picture used in this paper, however, allows for a complete understanding of the interplay between the global and local degrees of freedom at the transition lines.

\section{Phase diagram}

A schematic zero-temperature phase diagram for the chain is shown in Fig. 2. It is convenient to show this as a function of the shunt resistance $R_{Q} / R$ and the stiffness superconducting stiffness $K$ at various values of the conversion resistance $r$. [Although a fourth parameter, related to QPS fugacities, is really needed as well to exhibit the range of possible behaviors.] Here $R_{Q}$ is the quantum resistance for Cooper pairs

$$
R_{Q}=\frac{h}{4 e^{2}} \approx 6.5 k \Omega .
$$

For large $r, r>R_{Q}$, the phase fluctuations on each grain are large, and only the normal and $\mathrm{SC}^{\star}$ phases can exist. In this regime, the phase diagram is simple (Fig. 2) with the global transition between these phases at $K=K_{G} \approx 4$. This transition is driven by proliferation of quantum phase slips, is analogous to the classical Kosterlitz-Thouless transition in two dimensions, and has characteristic energy scale going to zero exponentially rapidly as the transition is approached, and a correlation length that diverges with the inverse of this energy scale.

For intermediate $r, R_{Q}>r>r_{c} \approx \frac{1}{2} R_{Q}$, all three phases exist, but the mixed transition between the FSC and normal phases is always driven by dipoles. The critical $R, R_{M}$, varies with $r$ and weakly with $K$ : it is close to when $r+\frac{1}{4}(R$ $\left.+\sqrt{R^{2}+4 r R}\right)=R_{Q}$. The energy scale goes to zero as a power of $\left|R-R_{M}\right|$ with an exponent $\mu$ that varies continuously with $r$. The characteristic length scale on the normal sideassociated with proliferation of individual dipoles - diverges as a power of $R-R_{M}$ with a continuously variable exponent that is not simply related to the that of the inverse energy scale. The FSC-SC ${ }^{\star}$ local transition is similarly driven by dipoles, and occurs when the same combination of $R$ and $r$, is close to $R_{Q}$. The energy scale similarly goes to zero with a continuously varying exponent.

For $r<r_{c} \approx \frac{1}{2} R_{Q}$, the phase diagram is far richer (Fig. 2), and qualitatively similar to the previously studied $r=0$ case of infinitely fast relaxation between the normal and super- 

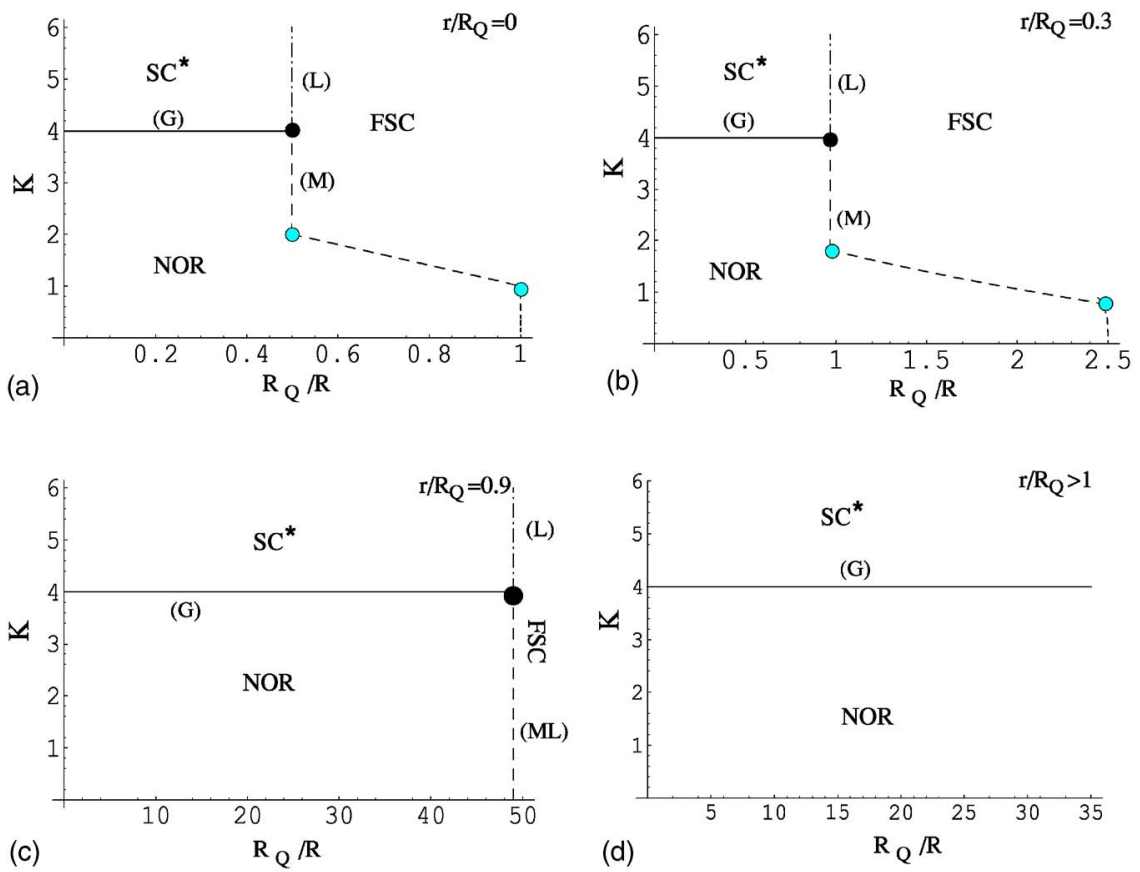

FIG. 2. (Color online) Schematic phase diagram of the quantum two fluid model of Fig. 1(c) as a function of the shunt resistance $R$ and the quantum superconducting stiffness $K=2 \pi \sqrt{\frac{E_{J}}{E_{C}}}$ for various values of the conversion resistance $r$. The phase boundaries between the FSC, $\mathrm{SC}^{\star}$, and NOR phases are of different nature: $(\mathrm{G})$ indicates global (solid line), (L) local (dashed-dotted line), and (M) mixed (dashed lines). These come together at a multicritical point (black dot). The FSC-NOR phase boundary has sections with three different characters, separated by bicritical points (gray dots). Most of the phase boundaries are derived from the strong Josephson limit (Sec. IV), but their positions will depend on the fugacity $\zeta$ of quantum phase slips, which should be another axis. For small $K$, the weak coupling expansion (Sec. V) is needed: that portion of the FSC-NOR boundary is inferred from it.

conducting electrons. The $\mathrm{SC}^{\star}$-normal and FSC-SC ${ }^{\star}$ transitions are similar to the intermediate $r$ regime discussed above. But the mixed-character phase transition between the FSC and normal phases is more complicated. For largeintermediate Josephson coupling $K_{B}<K<4$ with $K_{B} \leq 2$ depending on $r$ and $R$, the mixed transition is similar to that in the intermediate $r$ regime (above). In the limit of weak Josephson coupling - small $K$-its character is different. In this regime, the mixed transition is most easily thought of as being driven-from the normal phase-by Cooper-pair tunneling. It occurs when $R=R_{M} \approx R_{Q}-2 r$ with $R_{M}$ decreasing to this value as $K \rightarrow 0$. There is power law scaling of energy in the vicinity of this transition, with continuously variable exponents.

In some regime of parameters, the small coupling and large-intermediate coupling phase boundaries may join together: if they do so, it will be in an intermediate coupling regime that is far from all three phases and thus for which we have no controlled methods to study. At this point, it is not clear whether or not these two regimes can join continuously. Naively, their character, particularly the behavior of length scales, seems rather different. But it is possible that they are related and then could join continuously.

In the regime in which we can understand the behavior in terms of quantum phase slips, there is a section of the FSCnormal phase boundary which has different character than those discussed above. For $K<K_{B}$ but not too small-smallintermediate-direct proliferation of individual QPS (rather than their proliferation caused by dipole proliferation) drives the transition of the FSC phase. Although the behavior is more subtle and there are complicated crossovers, the asymptotic critical behavior is similar to the KosterlitzThouless transition between the $\mathrm{SC}^{\star}$ and NOR phases with exponentially vanishing energy scale and length scale diverging as the inverse of this, i.e., isotropic behavior. Surprisingly, the location of the transition is determined by a combination of the low energy properties of the superconducting and normal-i.e., dissipative-degrees of freedom. But how this changes measurable asymptotic properties we have not worked out.

In the low range of $r$ in which the mixed transition can have more than one character, the different sections of the phase diagram join together at two bicritical points. The weak-coupling power-law regime joins up to the smallintermediate coupling KT-like regime at a point that is neither near the $K=0$ nor the large $K$ regime and thus not amenable to study by the methods we use. But the other bicritical point at which the dipole driven and individual QPS driven segments of the phase boundary come together can be analyzed: we discuss it briefly in Sec. IV G.

The three phases come together at a multicritical point as shown in the figures. The behavior near this point involves crossover from the FSC to either the $\mathrm{SC}^{\star}$ or normal phase to the left of the nearly vertical phase boundary. The asymptotic critical behavior, however, is probably controlled by the $\mathrm{SC}^{\star}$ phase. We have not investigated this in detail.

We note that in the limit $r=0$ our phase diagram in the strong coupling limit coincides with that in Refs. 42 and 43. 
It differs somewhat from that observed using MC in Ref. 41, which could be understood as due to crossover effects discussed in Secs. IV and VII. In the weak coupling limit, our phase diagram agrees with that observed in Ref. 41 .

\section{Renormalization-group analysis}

The primary methods we use are RG analyses of the effective low energy action for the quantum two-fluid JJ chain model; both Coulomb gas and sine-Gordon representations of this action are derived and used in the various regimes. The zero-temperature phase diagram in various limits, and the nature of the quantum phase transitions more generally, are derived from these. The RG method naturally leads to detailed understanding of both the qualitative and the quantitative roles of various aspects of the model, for example, that the dissipation is irrelevant in the $\mathrm{SC}^{\star}$ phase and near the "global" normal-to-SC ${ }^{\star}$ phase transition. For the normal-toFSC transition, on the other hand, dissipation plays a key role by suppressing local fluctuations of the phase, and the characteristic length scale is the relaxation length between the superconducting and the normal fluids; as $r$ varies from zero and infinity, this length-scale changes from the intergrain spacing $a$ to infinity, although the low energy properties of the transition from the FSC to the $\mathrm{SC}^{\star}$ phase remains "local."

The RG flow equations can also be used to obtain the temperature dependence of various quantities, notably the resistivity close to the superconductor-to-normal transitions. In the vicinity of the global normal to $\mathrm{SC}^{\star}$ transition the dissipation plays little role and the temperature dependence of the measured total resistivity near this quantum KT transition has been analyzed by other authors. ${ }^{65,66}$ However, in the vicinity of the mixed normal to FSC transition, the behavior is strikingly different.

The RG analysis also gives information about crossovers and regimes of validity of the asymptotic behaviors. It is important to emphasize that over much of the parameter ranges, the superconductor-to-normal transitions are likely to be characterized by wide crossover regions (see Fig. 2) in which, for example, the resistivity can be almost temperature independent over extended temperature ranges. This may correspond to the "supermetallic" behavior (very low but finite resistance roughly temperature independent) in the vicinity of superconductor-to-normal transitions, that has been observed in several experiments in one dimensional ${ }^{52,67-70}$ and two dimensional systems. ${ }^{48,63}$

The RG can also be used to study finite size properties and effects of boundary conditions. For example, we show that when the RSJJ chain is connected to superconducting electrodes, at asymptotically low temperatures in the $\mathrm{SC}^{\star}$ regime, the appropriate measure of the dissipation that controls the location of the macroscopic superconductor-tonormal transition is the total shunting resistance, in contrast to the traditional picture that the local intergrain shunting resistance will control the macroscopic behavior (see also discussion, Sec. VIII). ${ }^{71}$

\section{Nanowires}

Late in the paper we go briefly from the realm of discrete grains and Josephson junctions to that of continuous super- conducting wires. This limit, to the best of our knowledge, was not discussed in previous work, although it is most relevant to experiment. For continuous superconducting wires, there is a subtle question about whether the FSC phase exists. The approximate model we use, leads to the conclusion that it does not. If this is correct, then at sufficiently low temperatures, the wires will always approach the $\mathrm{SC}^{\star}$ or the normal phase, but there can be wide regimes of crossover in which FSC-like behavior may be observable. But aspects that have been left out of the model, in particular aspects of charge discreteness and interference between quantum phase slips, may invalidate this conclusion. Preliminary indications are that these can stabilize the FSC phase. Its existence is thus left as an open question.

\section{MESOSCOPIC MODEL OF CHAIN OF GRAINS}

The system we study consists of identical mesoscopic superconducting grains that are connected by weak links which allow the flow of both Cooper pairs and normal electrons [Fig. 1(a)]. As discussed above, it is convenient to describe this system in terms of a two fluid model. The superfluid is transported by Cooper pairs tunneling between superconducting grains, and the normal fluid is transported by electrons through the shunting resistors. Each grain is thus considered as a double grain with superconducting and normal parts as shown in Fig. 1(c). The $S$ (superconducting) and $N$ (normal) parts of each grain experience the same electrostatic potential $\varphi$ but they will generally have different chemical potentials. (An analogous decoupling of the chemical potentials for the $S$ and the $N$ fluids near phase-slip centers in superconducting wires in the presence of a transport current was discussed in Refs. 72-74.)

The possibility of having different chemical potentials for the normal and superfluid components is a consequence of the mesoscopic size of the grains. The conversion resistance between the two fluids within a grain is inversely proportional to a positive power of the size of a grain (in the case of a uniform grain, $r$ is inversely proportional to the volume of the grain), and therefore it will provide significant dissipation only for small grain sizes. The dependence of $r$ on the size of a grain should be obtained from an appropriate microscopic model, which is beyond the scope of this paper. ${ }^{75}$

The sum of the electrostatic potential $\varphi_{i}$ and the chemical potentials $\mu_{N i}, \mu_{S i}$ of the $i$ th grain yield the total normal and superfluid electrochemical potentials $V_{N i}$ and $V_{S i}$ which will drive the currents.

Changes in the total charge on a grain modify its electrostatic potential via the capacitance $C$ while changes in the normal or superfluid charges on a grain correspondingly modify the chemical potentials with the coefficients the inverse compressibilities $D_{N}$ or $D_{S}$ of the $N$ and $S$ fluids on an individual grain; these compressibilities thus have the character of "quantum capacitances."76

When the electrochemical potentials of the $N$ and $S$ fluids on a grain differ, relaxation processes will occur to equilibrate the two components. The simplest form for such relaxation is an Ohmic conversion current $I_{N S i} .{ }^{59}$ 


$$
I_{N S i}=\frac{V_{N i}-V_{S i}}{r}
$$

where $r$ is the conversion resistance within a grain. We assume that $r$ remains finite even in the limit of zero temperature.

The charge relaxation time between superconducting and normal fluids on a grain is set by the $R C$ time of the effective circuit

$$
\tau_{N S}=r\left(D_{S}+D_{N}\right)^{-1}
$$

Therefore our assumption of an Ohmic conversion resistance is equivalent to assuming a form for the relaxation rate between the normal fluid and the superfluid.

The effective low-energy model of the system shown in Fig. 1(c) should include the charging energy for the grains, the Josephson coupling energy, and appropriate heat bath Hamiltonians for the shunting and conversion resistors. We now construct the appropriate Hamiltonian and thence obtain the corresponding quantum action.

We start with the charging energies. The electrochemical potentials for the superconducting and the normal fluids include contributions from both electrostatic and electrochemical "capacitances" [see Fig. 1(c)]

$$
\begin{gathered}
V_{S i}=\varphi_{i}+D_{S} Q_{S i}, \\
V_{N i}=\varphi_{i}+D_{N} Q_{N i},
\end{gathered}
$$

where $\varphi_{i}$ is the electric potential of grain $i$, and $Q_{S i}, Q_{N i}$ are the superconducting and the normal parts of the charge on grain $i ; D_{S}$ and $D_{N}$ are the inverses of the compressibilities of the $S$ and $N$ grains, respectively. In this paper we consider a simplified model in which only the self-capacitance of each grain $C$ is included. We can then write the electric potential as

$$
\varphi_{i}=\frac{1}{C}\left(Q_{S i}+Q_{N i}\right)
$$

We expect that including mutual capacitances between the grains $^{77,78}$ will not change the qualitative conclusions, although it may modify the energy scales involved.

By integrating the electrochemical potentials in Eq. (5) with respect to the charge, we obtain the charging part of the Hamiltonian. The resulting term is

$$
\begin{aligned}
\mathcal{H}_{Q}= & \frac{1}{2}\left(C^{-1}+D_{S}\right) \sum_{i} Q_{S i}^{2}+\frac{1}{2}\left(C^{-1}+D_{N}\right) \sum_{i} Q_{N i}^{2} \\
& +C^{-1} \sum_{i} Q_{S i} Q_{N i} .
\end{aligned}
$$

In order to write down the action for the dissipative and Josephson terms, we define phase-angles conjugate to the charges $Q_{S}$ and $Q_{N}$. For each grain $i$ the superconducting phase $\phi_{i}$ and the "normal phase" $\chi_{i}$ are defined via 37,59

$$
\left[Q_{N i}, \chi_{j}\right]=-i e \delta_{i j}, \quad\left[Q_{S i}, \phi_{i}\right]=-2 i e \delta_{i j}
$$

$$
\left[Q_{N i}, \phi_{j}\right]=0, \quad\left[Q_{S i}, \chi_{j}\right]=0 .
$$

The physical interpretation of $\chi_{i}$ follows from the observation that its time derivative gives the electrochemical potential of the normal fluid, by analogy with the Josephson relation for the superfluid. ${ }^{34,37,79}$

The Hamiltonian term arising from the Josephson tunneling between grains can be readily written in terms of $\phi_{i}$ :

$$
\mathcal{H}_{J}=-E_{J} \sum_{\langle i j\rangle} \cos \left(\phi_{i}-\phi_{j}\right),
$$

where the summation is over nearest-neighbor grains $i$ and $j$. $E_{J}$ is the Josephson coupling energy of the Josephson junctions, given by $E_{J}=\frac{\hbar}{2 e} I_{J}$ in terms of $I_{J}$, the critical current of the junctions.

The dissipative parts of the system can be described by heat bath Hamiltonians with appropriately chosen spectral functions. These are written as follows:

$$
\mathcal{H}_{\text {dis }}=\sum_{\langle i j\rangle} \mathcal{H}_{\text {bath }}\left(R, 2 \chi_{i}-2 \chi_{j}\right)+\sum_{i} \mathcal{H}_{\text {bath }}\left(r, \phi_{i}-2 \chi_{i}\right)
$$

We do not give the explicit form of $\mathcal{H}_{\text {bath }}$ here, but below provide the corresponding effective actions obtained after integrating out the heat-bath degrees of freedom. The crucial requirement for Ohmic heat baths is that their density of states is linear at low frequencies. Note that this is the case for particle-hole excitations of a Fermi liquid, one likely source of dissipation in dirty gapless superconductors especially near transitions to normal metallic behavior.

Combining Eqs. (7)-(10) we construct the imaginary time action and partition function for the RSJJ array of Fig. 1(c):

$$
Z=\int \mathcal{D} Q_{N} \mathcal{D} Q_{S} \mathcal{D} \phi \mathcal{D} \chi \exp (-S)
$$

$$
\begin{aligned}
S= & -\frac{i}{2 e} \sum_{i} \int_{0}^{\beta} d \tau Q_{S i} \dot{\phi}_{i}-\frac{i}{e} \sum_{i} \int_{0}^{\beta} d \tau Q_{N i} \dot{\chi}_{i} \\
& +\int_{0}^{\beta} d \tau \mathcal{H}\left(Q_{N i}, Q_{S i}, \phi_{i}, \chi_{i}\right), \\
& \mathcal{H}\left(Q_{N i}, Q_{S i}, \phi_{i}, \chi_{i}\right)=\mathcal{H}_{Q}+\mathcal{H}_{J}+\mathcal{H}_{\mathrm{dis}} .
\end{aligned}
$$

In the presence of Ohmic dissipation or an external current source, the phase variables $\phi_{i}$ and $\chi_{i}$ should be periodic at $\tau=0$ and $\tau=\beta$ with no phase twists by multiples of $2 \pi$ allowed: in contrast to simple nondissipative Hamiltonians, there is a physical distinction between $\phi_{i}-\phi_{j}=0$ and $2 \pi$ (see, e.g., the discussion in Sec. IIB of Ref. 59).

We can integrate out the $\left\{Q_{N i}\right\}$ and $\left\{Q_{S i}\right\}$, as these appear quadratically in Eq. (11), and obtain

$$
\begin{aligned}
& Z=\int D \phi D \chi \exp \left(-S_{\text {chain }}\right), \\
& S_{\text {chain }}=S_{Q}+S_{\text {dis }}^{r}+S_{\text {dis }}^{R}+S_{J},
\end{aligned}
$$




$$
\begin{gathered}
S_{Q}=\int d \tau \sum_{i} \frac{1}{\left(D_{N}+D_{S}+C D_{N} D_{S}\right)} \\
\times\left\{\frac{1}{2}\left[\dot{\phi}_{i}(\tau)-2 \dot{\chi}_{i}(\tau)\right]^{2}+\frac{C D_{S}}{2}\left(2 \dot{\chi}_{i}\right)^{2}+\frac{C D_{N}}{2} \dot{\phi}_{i}^{2}\right\}, \\
S_{\mathrm{dis}}^{r}=\beta \sum_{\omega_{n}}\left[\frac{\left|\omega_{n}\right|}{r}\left|\phi_{i}\left(\omega_{n}\right)-2 \chi_{i}\left(\omega_{n}\right)\right|^{2}\right] \\
S_{\mathrm{dis}}^{R}=\beta \sum_{\omega_{n}} \sum_{\langle i j\rangle} \frac{\left|\omega_{n}\right|}{R}\left|2 \chi_{i\left(\omega_{n}\right)}-2 \chi_{j}\left(\omega_{n}\right)\right|^{2} \\
S_{J}=-E_{J} \int_{0}^{\beta} d \tau \sum_{\langle i j\rangle} \cos \left(\phi_{i}-\phi_{j}\right),
\end{gathered}
$$

where Matsubara frequencies $\omega_{n}=2 \pi n T$ and Fourier transforms of the periodic functions $\phi_{i}(\tau), \chi_{i}(\tau)$ are used with $f\left(\omega_{n}\right) \equiv \int_{0}^{\beta} f(\tau) e^{i \omega \tau} d \tau$. In the limit of zero temperature, which we will primarily study, $\beta \Sigma_{\omega_{n}}$ is replaced by $\int \frac{d \omega}{2 \pi}$.

\section{A. Scales, parameters, and regimes}

There are several important energy and length scales in the mesoscopic model of a chain of RSJJ's, as well as several key dimensionless parameters. The superconducting energy scale is the Josephson coupling energy $E_{J}$. Competing with it are (twice) the charging energy of a grain

$$
E_{C}=\frac{(2 e)^{2}}{C}
$$

and the analogous nonelectrostatic portions of the energies of adding a normal electron

$$
E_{D N}=e^{2} D_{N},
$$

or a Cooper pair

$$
E_{D S}=4 e^{2} D_{S}
$$

to a grain. In most situations of interest, the energy scales $D_{N}$ and $D_{S}$ satisfy

$$
D_{N} \sim D_{S} \ll 1 / C,
$$

so that the electrostatic energy dominates. At low energies the $D$ 's drop out and only $C$ is important. The ratio of the Josephson to the charging energy then determines the dimensionless quantum phase stiffness

$$
K=2 \pi \sqrt{E_{J} / E_{C}} .
$$

In the absence of dissipation, the low temperature behavior is controlled by the parameter $K$. For large $K$, the phase differences between neighboring grains are small and the Josephson coupling can be approximated by $E_{J}\left(\phi_{i+1}-\phi_{i}\right)^{2} / 2$. This yields the conventional quadratic Hamiltonian for the superconducting degrees of freedom. With intergrain spacing $a$, the characteristic velocity of the phase fluctuations is

$$
c=\frac{a \sqrt{E_{J} E_{C}}}{\hbar},
$$

which corresponds to the Mooij-Schön velocity. ${ }^{80,81}$ In this superconducting phase, the correlations decay as powers of distance and imaginary time with an exponent proportional to $K$.

The dissipation can be parametrized by the dimensionless resistances, $R / R_{Q}$ and $r / R_{Q}$ where we used the quantum of (Cooper pair) resistance

$$
R_{Q}=\frac{2 \pi \hbar}{4 e^{2}}
$$

these dimensionless resistances are key control parameters. When $R \ll r$, the effects of the two resistances- $R$ 's in series and $r$ 's in parallel—becomes comparable at a length scale

$$
\lambda_{Q} \approx a \sqrt{r / R}
$$

which plays an important role. In this small $R$ regime, the dimensionless measure of the dissipation is

$$
\alpha \approx \frac{R_{Q}}{2 \sqrt{r R}} .
$$

When $R$ is comparable to or larger than $r$, the characteristic length and dissipation measure have more complicated dependencies. In particular,

$$
\alpha=\frac{R_{Q}}{\sqrt{R^{2}+4 r R}} ;
$$

as discussed in the next section, this can be understood from electrodynamical considerations.

The dissipative energy scale is determined by the competition between the compressibilities (which did not directly affect the superconducting degrees of freedom) and the resistances. The effective resistance $R^{*} \sim \min (r, R)$ and effective capacitance

$$
C_{S N}=\frac{1+C D_{S}}{D_{N}+D_{S}+C D_{N} D_{S}} \approx \frac{1}{D_{N}+D_{S}}
$$

together give the characteristic relaxation time that determines the energy scale $T^{*}$ parametrizing the coupling between the normal and superconducting currents

$$
T^{*}=\frac{\hbar}{R^{*} C_{S N}} .
$$

The energy scale $T^{*}$ can also be written in terms of the other energy scales. From Eq. (23), one finds that $T^{*}$ is proportional to the escape time of normal electrons from the grain, i.e., to the Thouless time of the grain. It is thus very large for macroscopic grains, but its existence is an essential property of the mesoscopic physical content of our model.

At temperatures higher than $T^{*}$, the dissipation across separate junctions is effectively decoupled. In contrast, for $T \ll T^{*}$, the interactions between dissipation across different junctions and within different grains are important. These interactions are crucial for the quantum dynamics. At low energies, the existence of the compressibilities thus matters 
crucially, although the values of the $D$ 's do not.

Before proceeding with more sophisticated analyses, it is instructive to consider the linearized dynamics in the presence of dissipation. As all the terms in the model action except the Josephson coupling are quadratic, we can integrate out all but the superconducting phase $\phi$ and expand the Josephson coupling energy about zero phase difference. This results in a rather messy form of the action that is given in the Appendix. In the limit of low frequencies and long wavelengths the dissipative effects are negligible and all that matters are the conventional superconducting parts of the action including the suppression of imaginary-time changes of the phase by the inverse of the total effective charging energy. These give rise to the simple phase modes discussed above. Nevertheless, the fact that the short wavelength fluctuations are controlled by the dissipation, makes the terms that appear negligible at long wavelengths also important for the quantum dynamics and thence the phase diagram.

In the absence of dissipative effects, the mean square fluctuations of the phase difference between neighboring grains would be inversely proportional to the quantum stiffness $K$. However, these fluctuations are dominated by wavelengths of order the intergrain spacing, and frequencies of order the Josephson junction plasma frequency (proportional to $c / a$ ). Thus, in actuality, the modes that dominate the phase fluctuations will be affected by much of the details of the high frequency dynamics, including the dissipation and the cutoff frequencies of both the dissipative and the superconducting degrees of freedom. We will often crudely approximate these by a high-frequency cutoff $\Omega_{0}$. The short wavelength processes also control the action of a quantum phase slip via properties of its core, including its space-time size and "shape;" in particular, the "fugacity" of phase slips will be exponentially small when their core action is large.

Because of the importance of the high energy physics, it is not obvious what the significant dimensionless parameters are, beyond the obvious one discussed above, nor what role these might play at low energies. In practice, whether a chain of grains is in "strong" or "weak" Josephson coupling regime will be determined by many properties. Thus we will use these terms loosely to describe various regimes in which the behavior simplifies: small QPS “fugacity," $\zeta$ for strong coupling, and small Cooper pair tunneling rates for weak coupling. Care must thus be exercised in considering phase diagrams of more explicit models as changing one parameter can result in, for example, changing both the low frequency dissipation, and the fugacity of quantum phase slips.

To simplify discussions of phase boundaries and the behavior near them, we will generally consider tuning the shunt resistance $R$ and the strength of the Josephson coupling, either $E_{J}$ itself or the QPS fugacity as a proxy for this. The phase diagrams in general need to be considered as functions of both $r$ and parameters related to other high energy processes as well.

\section{B. Circuit analysis}

In the analysis of the two junction problem in Ref. 59 we showed that electrical circuit properties determined the (low-

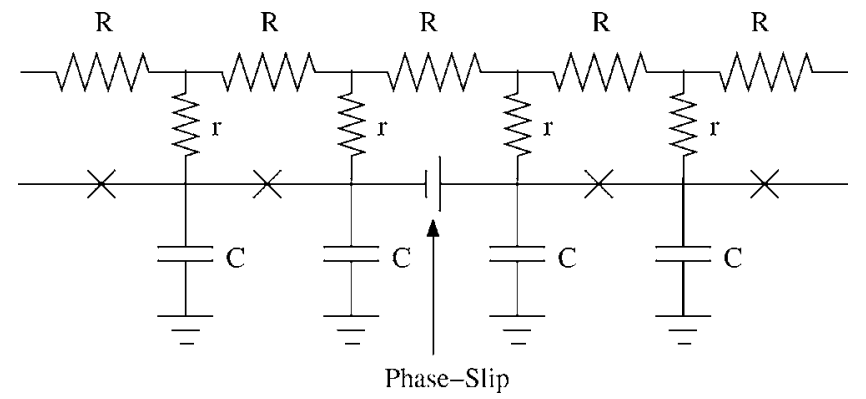

FIG. 3. A phase slip creates a potential drop across the junction, which propagates currents in the rest of the chain.

est order) RG flows in various limits. Before proceeding further, we analyze the linearized electrodynamics of the chain model to gain insight into its behavior.

The basis of the circuit approach is the following simple interpretation of the dissipation-driven transition in a single resistively shunted Josephson junction. ${ }^{34,37}$ When the junction is in the insulating state, the Cooper pairs are localized on the electrodes, and the phase difference across the junction is uncertain due to proliferation of quantum phase slips (QPS). In the opposite limit when the junction is superconducting, the phase difference between the grains is well defined and, phase slips will not occur on long time scales. A phase slip across the junction causes a voltage burst with the Josephson relation giving

$$
\int d t V(t)=\frac{h}{2 e}=\frac{2 \pi \hbar}{2 e}
$$

which leads to a current flow in the shunting resistor $R_{S}$. It turns out that the superconducting state of the junction is stable only when a single QPS would cause a charge flow, $\Delta Q$, which is more than a Cooper pair charge $2 e$. From Ohm's law we then obtain $\Delta Q / 2 e=R_{Q} / R_{S}$, so superconductivity will be observed when $R_{S}<R_{Q}$. A related analysis from the insulating state in terms of Cooper pair tunneling events can be used to argue for the same condition $R_{S}>R_{Q}$ stabilizes the insulating state.

\section{Cooper pair tunneling}

In the normal phase the grains are only coupled resistively, although they can exhibit superconducting correlations within each grain. Thus we can consider a putative Cooper pair tunneling from one grain to the next. This is very similar to the single junction case, except that now the total shunt resistance is $R+2 r$ because of the contributions of the conversion resistances in each grain (see Fig. 3). We are interested in the effect of this tunneling on the SC phase difference $\phi \equiv \phi_{i+1}-\phi_{i}$ across the junction. By the Josephson relation, the change in $\phi$ is given by

$$
\begin{aligned}
\Delta \phi & =\int d t \frac{d \phi}{d t}=\int d t \frac{2 e V(t)}{\hbar}=\frac{2 e V(\omega=0)}{\hbar} \\
& =\frac{2 e I(\omega=0) Z(\omega=0)}{\hbar}=\frac{2 e \Delta Q Z_{S}}{\hbar}=2 \pi \frac{Z_{S}}{R_{Q}}
\end{aligned}
$$

with $I$ the current, $\Delta Q=2 e$ the charge transferred, and $Z$ the 
impedance. Thus we see that the phase difference between the grains will change by more than or less than $2 \pi$ according to whether the shunting impedance $Z_{S}=R+2 r$ is more or less than $R_{Q}$. Since the transition from the normal phase to the FSC phase in which each grain has a well-defined SC phase is via this local process, it is not surprising that the condition for this to occur involves this combination. As we shall see, the behavior is in fact more subtle due to the effects of multiple Cooper pair tunnels on each other.

It is also instructive to consider the classical action associated with the tunneling of a Cooper pair. Since the phase and the Cooper pair number are canonically conjugate, the action will be given by

$$
\begin{aligned}
S_{p t} & =\hbar \int \frac{Q}{2 e} d \phi=\int d t Q(t) V(t)=\int \frac{d \omega}{2 \pi} Q(\omega) V(-\omega) \\
& =\int \frac{d \omega}{2 \pi} \frac{I(\omega)}{-i \omega+0} V(-\omega)=\int \frac{d \omega}{2 \pi} \frac{Z(\omega)|I(\omega)|^{2}}{(-i \omega+0)}
\end{aligned}
$$

note the integral of the potential energy appearing. Since in the limit of zero frequency the impedance is purely real and $I(\omega) \rightarrow 2 e$, the zero frequency part of this integral gives $\pi \hbar Z_{S} / R_{Q}$. The finite frequency parts will be negligible if the transfer is slow; otherwise they will decrease the action so that

$$
S_{p t} \leq \frac{\pi \hbar(R+2 r)}{R_{Q}} .
$$

In imaginary time, the $1 /(-i \omega+0)$ becomes $1 /|\omega|$ and the integral over frequencies diverges logarithmically at low frequencies or low temperatures. It is exactly the competition between this logarithmic action and the quantum "entropy" - $\log$ of the range of imaginary time $\hbar / T$-in which the event can occur that determines whether the junction is superconducting, as we shall see. In real time, the significance of Eq. (28) is not clear, in particular, whether $\exp (i S / \hbar)=\exp (i \pi)$ is significant as far as whether or not Cooper pair tunnels can destructively interfere, and if they can, thereby suppressing superconductivity for large shunt resistance.

\section{Effective shunting resistance in the strong Josephson coupling limit}

We now turn to the superconducting phase in which the Josephson junctions are all superconducting. Consider a phase slip across one junction in the chain. By analogy with the single junction case, one would guess that the relevant quantity is the low frequency limit of the impedance $Z(\omega)$ of the circuit parallel to it. This parallel circuit involves all the other superconducting junctions and the resistors. The total dissipation now comes from both the network of the Ohmic resistors and the Josephson junction chain itself.

The parallel shunting impedance of a junction in the chain splits into two contributions: the resistance of the network of resistors $r$ and $R$, and the impedance of the "telegraph line" of the chain. The first part is self explanatory; let us denote it as $R_{\text {eff }}^{s}$. The second piece requires an explanation. When the Josephson coupling is strong we can assume that Josephson

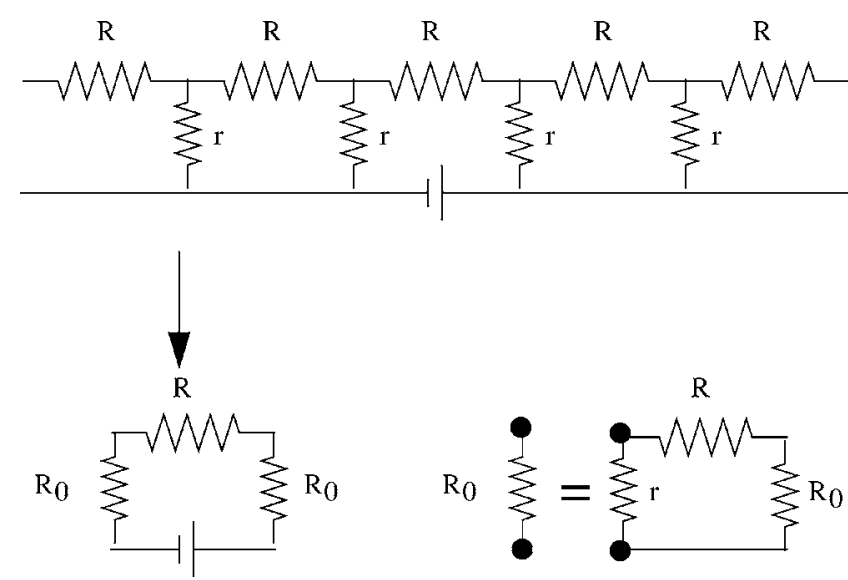

FIG. 4. The resistor network parallel to a single phase slip. The shunting resistance can be calculated using the "infinite resistor chain" trick.

junctions are superconducting except for the times in which they exhibit a phase slip. A superconducting Josephson junction has the impedance of a solenoid with the ("kinetic") inductance $L=\hbar /(2 e)^{2} E_{J}^{-1}$. Therefore the line of junctions and capacitors resembles a line of solenoids and capacitors, a simple model of a telegraph line. When a phase slip occurs, it creates a short-lived voltage drop on the junction (see Fig. 3). This pulse has two effects; the first is a dc current that flows through the shunt and conversion resistors $R$ and $r$ and the second effect is sending plasma waves through the line of junctions and capacitors. The latter also behaves as a resistance as it corresponds to energy being carried away by plasmons. Let us denote the effective resistance describing this mechanism of dissipation as $R_{\text {eff }}^{P}$. The two mechanisms of dissipation separate at low frequencies, since the resistive contribution comes from local currents which decay rapidly, whereas the plasmon contributions arises from waves propagating at speed $c$. Hence, the two mechanisms are totally out of phase with each other at low frequencies.

The effective resistance a phase slip feels due to the plasmons is

$$
R_{\mathrm{eff}}^{P}=2 \sqrt{\frac{L}{C}}=2 R_{Q} \frac{1}{2 \pi} \sqrt{E_{C} / E_{J}}=\frac{2}{K} R_{Q}
$$

(see, for instance, Appendix 5D of Ref. 82). Note that $R_{\mathrm{eff}}^{P}$ include contributions from the telegraph lines on both sides of the given junction.

The resistance $R_{\text {eff }}^{s}$ can be found easily using the construction in Fig. 4. In the figure it is shown that the shunting resistance can be broken into three resistors in series, where the semi-infinite ladder of resistors $r$ and $R$ is replaced by an effective resistor $R_{0}=\frac{-R+\sqrt{R^{2}+4 r R}}{2}$. We thus have an effective shunting resistance of the network of Ohmic resistors

$$
R_{\text {eff }}^{s}=2 R_{0}+R=\sqrt{R^{2}+4 r R}=\frac{R_{Q}}{\alpha} .
$$

The total impedance $Z_{S}=Z(\omega=0)$ shunting a junction in the fully superconducting phase (FSC) is thus given by the two contributions in parallel: 


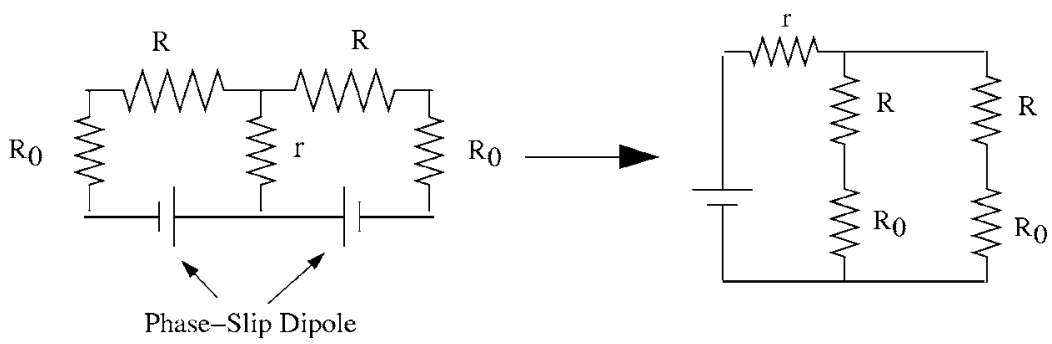

FIG. 5. Effective circuit for a phase-slip dipole with $s=1 . R_{0}$ is the effective resistance of a semi-infinite resistor ladder as given above Eq. (30). The dissipation seen by a dipole has no contribution from plasma waves at low frequencies.

$$
\frac{R_{Q}}{Z_{S}}=\frac{1}{2} K+\alpha .
$$

As we shall see, this combination controls the action of QPS in the FSC phase. From analogy with the single RSJJ case, we would expect that the FSC phase would become unstable to QPS when the effective inverse shunting resistance equals $R_{Q}$. As above for Cooper pair tunneling, we can see that the charge transferred associated with the quantum phase slip of $2 \pi$ is $V(\omega=0) / Z_{S}=2 e R_{Q} / Z_{S}$. Again by analogy with the Cooper pair tunneling, we can consider the action associated with the phase slip, finding that this is $\leq \pi \hbar R_{Q} / Z_{S}$. Thus we would guess that the condition for suppression of QPS tunneling is when

$$
\frac{R_{Q}}{Z_{S}}=\frac{K}{2}+\alpha \geq 1 .
$$

However, this analogy reflects only the local physics of phase slips and leads to the wrong condition: nevertheless, as we shall see, the correct condition involves the same combination.

In Sec. VII we discuss a region of parameter space in the NOR phase which we call a quasimetallic regime. In this region the measured resistance of the $\mathrm{JJ}$ chain first slowly drops as the temperature is lowered. But then, when a crossover temperature is reached, the resistance takes a sharp upturn (see Fig. 12). This effect occurs in the region of the NOR phase where the (wrong) local condition for the stability of the FSC phase to single phase slips [Eq. (32)] is fulfilled, but the FSC is unstable against dipoles.

To fully understand the effects of QPS, we also need to consider phase slip dipoles in which a phase slip occurs on one junction simultaneously with a phase slip of the opposite sign on another junction-say $s$ grains away. In the simplest case $s=1$ this has the effect of slipping the phase of one grain relative to the rest of the system to which it is coupled. More generally, $s$ dipoles slip the phase of $s$ consecutive grains relative to the rest of the chain. Because the effects of the two opposing phase slips cancel at long length scales, there will be no contribution to the effective shunting impedance from plasmons: it will be entirely dissipative. Considering all but the two junctions across which the slips occur, to be superconducting and thus short circuits at low frequencies, the effective shunt conductance between a row of $s$ grains and the rest of the chain is found to be Fig. 5,

$$
\frac{R_{Q}}{R_{\text {dipole }}^{S}}=2 \alpha\left(1-w^{S}\right),
$$

where

Of particular importance is the resistance between one grain and the rest of the chain that shunts the two (parallel) Josephson junctions. This is $r+\frac{1}{4}\left(R+\sqrt{R^{2}+4 r R}\right)=R_{Q} / \beta$ with $\beta$ $=2 \alpha(1-w)$ (see Fig. 5).

When $R \ll r . \alpha \approx 1 / 2 \sqrt{r R}, \beta \approx R_{Q} / r$, and $w \approx 1-\sqrt{R / r}$ so that the effective shunt resistances of strings of $s$ grains only separates into two resistors in parallel-one at each end-for large $s>\lambda_{Q} / a \approx \sqrt{r / R}$.

\section{3. $\mathrm{SC}^{\star}$ phase and dipole proliferation}

In the FSC state individual junctions have well defined phase differences so that the above calculations are relevant. But in the $\mathrm{SC}^{\star}$ phase the superconducting phases of the individual grains fluctuate strongly enough to decouple the superconductivity from the resistive shunts. In this case the energy of phase slips is primarily dissipated by the plasmons and the relevant impedance becomes just the $R_{\text {eff }}^{P}=2 R_{Q} / K$ with $K$ the dimensionless superconducting quantum stiffness.

A well known result is that a Josephson junction in its insulating state seems like a capacitor (see, for instance, Appendix 5E of Ref. 82). For dipole proliferation, the relevant junction is the combined junction from one grain to the rest of the system. To show this we first note that the effective capacitance of a Josephson junction when phase slips proliferate describes the electrical response of the "plasma" of phase slips. This effective capacitor is charged whenever current tries to cross the junction itself. For a dipole, the relevant current is the current that tries to leave the horizontal Josephson-junction line in Fig. 6. The current leaving the junction line into the vertically drawn wire is the only current that interacts with phase slip dipoles, hence the effective capacitor that appears when dipoles proliferate can get charged only by the current associated with $I_{\text {dipoles }}$ as in the Fig. 6. This effective capacitance thus occurs between the superconducting and normal parts of a junction, and it is in series to the finite conversion resistance which is important at low frequencies- $\omega \ll T^{*} / \hbar$. When dipoles proliferate, they effectively block the low-frequency conversion, as the effective capacitance of the dipoles dominates.

The induced capacitance due to dipoles blocks the $N-S$ conversion in each grain, and thus screens the dissipative interaction between phase slips: at low frequencies, this is essentially equivalent to setting $r \rightarrow \infty$. However, the plasmon interaction is a dynamic effect. As can be seen from Fig. 6 , the dipole-induced capacitance renormalizes the self capacitance $C$ of each grain. Thus the plasmon interaction sur- 


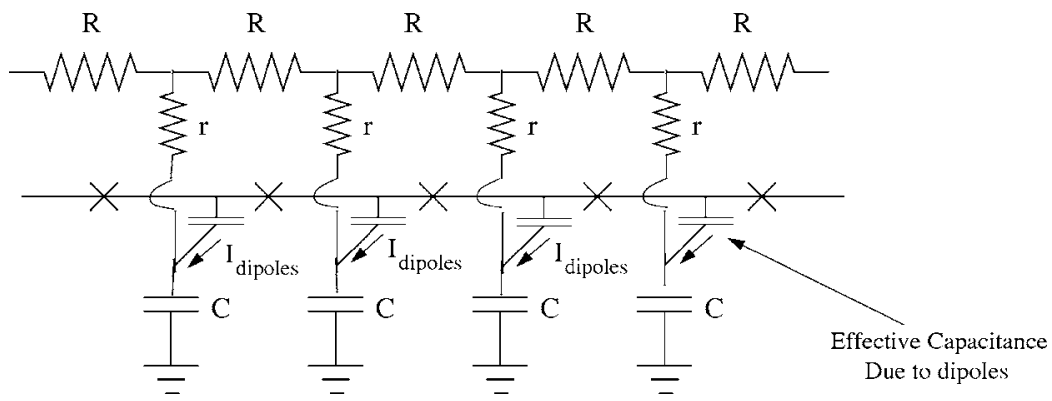

FIG. 6. Phase-slip dipoles create an effective capacitance which inhibits current from leaving the line of Josephson junctions. This capacitance screens the normal-to-superconductor conversion resistance $r$. But it only renormalizes the plasmawave dissipation via the change of the total effective capacitance of each grain.

vives the proliferation of dipoles, albeit with a renormalized plasma speed. These results are formally derived in Sec. IV $B$ from the effective action of the chain.

Since dipoles disconnect the $N-S$ conversion, in the $\mathrm{SC}^{\star}$ phase, the effective shunting resistance that is felt by a phase slip in the $\mathrm{JJ}$ chain is just that from the renormalized plasmon impedance. Therefore,

$$
\frac{R_{Q}}{R_{\text {total }}^{s}}=\frac{K}{2} .
$$

$R_{\text {total }}^{s}$ is the effective local dissipation in the $\mathrm{SC}^{\star}$ phase. One would expect, in analogy to the single junction that when

$$
R_{Q} / R_{\text {total }}^{S}<1
$$

the $\mathrm{SC}^{\star}$ phase would be unstable to QPS proliferation. As we shall see, the relationship between this shunting impedance and the stability of the SC ${ }^{\star}$ phase to QPS, is different than that for a single junction, essentially due to the "entropy" of the translational freedom of the QPS. Nevertheless, as in the case of the FSC-NOR QPS-driven transition, the local condition in Eq. (36) demarcates the regime with nonmonotonic temperature dependence of the resistivity. In the region of the NOR phase where condition (36) applies, there will be a low-energy crossover between a decreasing resistance as temperature decreases, to insulating behavior (see Sec. VII).

\section{STRONG JOSEPHSON COUPLING LIMIT}

We now turn to the analysis of the low energy properties of the chain, going well beyond the linear electrodynamics discussed in the previous section. We first derive the quantum-phase-slip Coulomb-gas representation of the action (Sec. III A) and transform it into a sine-Gordon representation (Sec. III B). This enables analysis of the phases of the JJ chain (Sec. III C), and an RG approach for the phase diagram (Sec. III D).

\section{A. Quantum phase slips and representations of the partition function}

In the limit of strong Josephson coupling, the phase differences between neighboring grains will predominantly be localized in the vicinity of the minima of the Josephson potential which occur at $\phi_{i}-\phi_{j}=2 \pi n_{i j}$, with $n_{i j}$ an integer. Occasionally, however, the phases will depart from their classical superconducting form, and tunnel between neighboring minima with different $n_{i j}$ : these events are quantum phase slips (QPS). Note that as discussed in the section on param- eters, strong coupling will in practice be defined by the rareness of QPS which depends on the high energy physics as well as the dimensionless Josephson coupling $K^{2} \propto E_{J} / E_{C}$.

Physically, QPS at zero temperature are caused by the charging energy [in Eq. (12)] not commuting with the Josephson potential. Quantum phase slips correspond to vortexlike phase configurations in the space- $\tau$ (imaginary-time) plane, while in real time they involve the launching of one dimensional plasma-waves (plasmons) through the chain.

At low temperatures, quantum phase slips may destroy superconducting coherence in the JJ array, and their potential role in this way makes them the basic excitations in terms of which the low energy physics can most readily be described when the local superconductivity is "strong." We can thus try to expand in the QPS fugacity in this strong Josephson coupling limit. As we shall see, one also has to consider phase slip dipoles: bound pairs of QPS of opposite signs.

\section{Coulomb gas representation}

To analyze the effects of QPS in the partition function, it is convenient to use a Villain transformation. As usual first writing

$$
\begin{aligned}
& \exp \left(\int d \tau E_{J}\left\{\cos \left[\phi_{i+1}(\tau)-\phi_{i}(\tau)\right]-1\right\}\right) \\
& \quad \approx \sum_{\left\{\mu_{i}(\tau)\right\}} \exp \left\{-\int d \tau \frac{E_{J}}{2}\left[\phi_{i+1}(\tau)-\phi_{i}(\tau)+2 \pi \mu_{i}(\tau)\right]^{2}\right\} .
\end{aligned}
$$

The Villain transformation breaks the cosine function of phase differences down to a sum over its troughs with $\mu_{i}(\tau)$ an integer valued function labeling the troughs in the Josephson potential of the junction between the $(i+1)$ th and $i$ th grains. A phase slip on the $j$ th junction corresponds to a sudden change of $\mu_{j}$ by \pm 1 , thus we can write it in terms of a density of discrete QPS:

$$
\rho_{\mathrm{QPS}}(j, \tau)=\frac{\partial \mu_{j}(\tau)}{\partial \tau}=\sum_{m} p_{m} \delta_{j, j_{m}} \delta\left(\tau-\tau_{m}\right),
$$

with the $m$ th QPS with "charge" $p_{m}= \pm 1$ occurring at imaginary time $\tau_{m}$ and on junction $j_{m}$. Periodic boundary conditions on the original phases $\phi_{i}(\tau=0)=\phi_{i}(\tau=\beta)$ imply an integrated neutrality condition for each junction: 


$$
\int_{0}^{\beta} \rho_{\mathrm{QPS}}(j, \tau) d \tau=0, \quad \forall j
$$

(this neutrality condition does not apply to time slices though $^{83}$ ).

Using Eq. (37) in the partition function, (12), makes the action quadratic in $\phi_{i}$ as well as in the other fields, all of which can be integrated out to obtain the partition function solely in terms of the QPS configurations. The action of a set of $N$ QPS with integer charges $\left\{p_{m}\right\}$ at space time locations $\left\{\left(x_{m}=a j_{m}, \tau_{m}\right)\right\}$, with (integer) $j_{m}$ labeling junctions, has the form

$$
S_{N}\left(\left\{p_{m}\right\}\right)=\frac{1}{2} \sum_{m \neq n}^{N} p_{m} p_{n} G\left(x_{m}-x_{n}, \tau_{m}-\tau_{n}\right),
$$

where $G(x, \tau)$ is the interaction between phase slips. The QPS partition function is then

$$
Z_{\mathrm{QPS}}=\sum_{\left\{p_{m}\right\}} \zeta^{N} \exp \left[-S_{N}\left(\left\{p_{m}\right\}\right)\right]
$$

with $\zeta$ the fugacity of QPS; $\zeta$ has units of frequency: in the absence of interactions between them, it would be the rate at which phase slips of each sign occur across a single junction. Its bare value is obtained by considering the action of the instanton which describes the short time motion of the phase variables following a phase slip in $\mu_{i}$. The sum in Eq. (41) is over distinguishable QPS configurations with the restriction that for each junction the total QPS charge is zero-a neutrality condition. Note that strictly speaking, because of this local neutrality, with $m$ and $n$ on different junctions $G\left(x_{m}\right.$ $\left.-x_{n}, \tau_{m}-\tau_{n}\right)$ is only the finite part of the interaction: the infinite parts that would arise if the local neutrality condition were not satisfied have been subtracted out.

The full form of the interaction between the QPS is very complicated and has several different regimes. As discussed in the Introduction, the temperature (energy) scale that arises from the dissipation and the finite size of the grains $T^{*}$ [see Eq. (24)] plays an important role. At temperatures higher than $T^{*}$ dissipation on separate junctions is effectively decoupled. By contrast, at low temperatures $T<T^{*}$, there is considerable interaction between QPS on neighboring junctions: we focus here and henceforth on this low temperature regime.

\section{Low temperature limit}

In the low temperature limit $T \ll T^{*}$ [for the definition of $T^{*}$ see Eq. (24)], the effective interactions between QPS is given by

$$
G(x, \tau) \approx K \ln \frac{\tau_{0}}{\sqrt{x^{2} / c^{2}+\tau^{2}}}+\alpha e^{-|x| / \lambda} Q \ln \frac{\tau_{0}}{|\tau|},
$$

where the quantum stiffness $K=2 \pi \sqrt{E_{J} / E_{C}}$ (assuming $\left.D_{N, S C} C \ll 1\right)$, the Mooij-Schön velocity $c=a^{\frac{\sqrt{E_{J} E_{C}}}{\hbar}}$, and the strength of the dissipative interactions

$$
\alpha=\frac{R_{Q}}{\sqrt{R^{2}+4 r R}},
$$

were all introduced earlier, and the characteristic length scale is

$$
\lambda_{Q}=\frac{a}{\ln (1 / w)}
$$

with

$$
w=1+\frac{R-\sqrt{4 r R+R^{2}}}{2 r}
$$

which is approximately $1-\sqrt{R / r}$ when $R \ll r$. The origin of $\lambda_{Q}$ is the decay length of currents in the resistive network shown in Fig. 4, and can also be inferred from the continuum equations (1).

The discreteness of the chain is important even at large length scales, particularly when $\lambda_{Q}$ is comparable to $a$. The strength of the dissipative interaction arises from

$$
\alpha=\int_{|k|<k_{m}} \frac{d k}{2 \pi} \frac{R_{Q} a}{r(2-2 \cos k a)+R} .
$$

This yields simply the inverse of the shunt resistance for a single junction, as found in Sec. II B. [In the continuum approximation with a sharp momentum space cutoff at $\pi / a$ the QPS interaction would yield $\alpha=\alpha \frac{2}{\pi} \arctan \left(\pi \sqrt{\frac{r}{R}}\right)$ : this is close to Eq. (43) over the whole range but does not correctly correspond to the shunt resistance. Similarly, in the continuum approximation, $\lambda_{Q}$ would always have its asymptotic form $a \sqrt{r / R}$.]

The cutoff for the plasma interaction is given by $\tau_{0}$ $\sim a / c=\hbar / \sqrt{E_{J} E_{C}}$. The cutoff for the dissipative interaction in the strong coupling limit, on the other hand, is $\tau_{0}$ $\sim \min \left\{\hbar R_{Q} /(R+2 r) E_{J}, \hbar / \sqrt{E_{J} E_{C}}\right\}$. When $R+2 r \gg R_{Q}$ the cutoff time for the local interaction is lower than that for the isotropic plasmon-related interaction. This may give rise to additional crossovers in the temperature range $R E_{J} / R_{Q}>T$ $>\sqrt{E_{J} E_{C}}$. However, we will only analyze the Coulomb-gas action (42) for energy scales and temperatures $\Omega<\sqrt{E_{J} / E_{C}}$.

The two logarithmic interactions between QPS in Eq. (42) have very different physical origins. The first part, which is isotropic in space-time, is present for $(1+1)$-dimensional $X$ - $Y$ models even in the absence of dissipation; it gives rise to a quantum Kosterlitz-Thouless transition, as originally discussed by Bradley and Doniach ${ }^{77}$ (see also Ref. 84). The second term in Eq. (42) originates from the Ohmic dissipation. It is logarithmic in the time separation of phase slips. Only the limit $r=0 \quad\left(\lambda_{Q}=0\right)$ has, to our knowledge, been analyzed previously. ${ }^{42,43,45}$ In this limiting case the dissipative interaction of Eq. (42) takes the form

$$
G_{\text {local }}(x, \tau)=a \delta(x) \frac{1}{R} \ln \frac{\tau_{0}}{|\tau|} .
$$

More generally, for nonzero $r$, the length scale $\lambda_{Q}$ is the range of normal currents that are induced when a phase slip occurs and thus, effectively, the dissipative size of a QPS: $\lambda_{Q}$, controls the exponential fall-off with spatial separation of 
the dissipative interactions between QPS's. Not surprisingly, $\lambda_{Q}$ plays a particularly important role in the physics of finite length chains. ${ }^{71}$

\section{B. Sine-Gordon representation}

From the Coulomb gas partition function, Eq. (40), we derive the sine-Gordon representation of the strong Josephson coupling limit. The Coulomb gas representation provides an expression for the probabilities of specific configurations of phase slips, whereas the sine-Gordon representation is more amenable to a renormalization group analysis. We introduce two separate Hubbard-Stratonovich fields, $\theta_{j}$ and $\psi_{j}$ located on the junctions between the grains $(j, j+1)$, in order to decouple the two contributions to the interactions between QPS of Eq. (42) (for details see Appendix 5A.2 of Ref. 82): in terms of the physical variables, $\psi$ decouples the normal degrees of freedom, and $\theta+\psi$ the superconducting degrees of freedom. This transformation yields the dual action of the model of Eqs. (12):

$$
\begin{aligned}
S_{\text {dual }}= & \int_{0}^{\beta} d \tau \frac{1}{4 \pi a^{2} \kappa} \sum_{i}\left(\theta_{i+1}-\theta_{i}\right)^{2} \\
& +\int_{0}^{\beta} d \tau \frac{1}{4 \pi J} \sum_{i}\left(\partial_{\tau} \theta_{i}\right)^{2}+\frac{\beta}{2 \pi} \sum_{\omega_{n}} \frac{\left|\omega_{n}\right|}{2}\left\{\frac{R}{R_{Q}} \sum_{i}\left|\psi_{i, \omega_{n}}\right|^{2}\right. \\
& \left.+\frac{r}{R_{Q}} \sum_{i}\left|\psi_{i+1, \omega_{n}}-\psi_{i, \omega_{n}}\right|^{2}\right\}-\zeta \int_{0}^{\beta} d \tau \sum_{i} \cos \left(\theta_{i}+\psi_{i}\right) .
\end{aligned}
$$

The effective stiffnesses are

$$
\kappa \equiv K / c=\frac{2 \pi \hbar}{a E_{C}}
$$

and

$$
J \equiv K c=\frac{a E_{J}}{2 \pi \hbar} .
$$

Phase-slip dipoles. In our recent work on pairs of shunted Josephson junctions ${ }^{85}$ we demonstrated the important role of QPS dipoles: these are instantons consisting of two opposite sign QPS that occur almost simultaneously on nearby junctions. The simplest phase-slip dipole, on neighboring junctions, is thus an event in which the phase of a single grain slips by $2 \pi$ relative to the chain. In nondissipative $X Y$ models such dipoles disappear when short wavelength fluctuations are integrated out isotropically in space and time, and the closely spaced dipoles act only to renormalize the stiffness $\kappa$ and thereby the interaction between other QPS.

For granular systems with dissipation, the physics on the scale of the grains plays an essential role, and a better approach is to perform the RG procedure in time only. This means that dipoles remain as independent degrees of freedom and should be considered explicitly along with individual QPS. (Although it is clumsy to do so, we will show later that the results for the isotropic Kosterlitz Thouless transition can be recovered from this anisotropic RG.) Di- poles can be included in the sine-Gordon representation by adding the following term to the action

$$
\begin{aligned}
& S_{\text {dipole }} \\
& \qquad=\sum_{s=1}^{\infty} \eta_{s} \sum_{i} \int d \tau \cos \left\{\left[\theta_{i}(\tau)+\psi_{i}(\tau)\right]-\left[\theta_{i+s}(\tau)+\psi_{i+s}(\tau)\right]\right\} \\
& =\sum_{i} \sum_{s} \int d \tau \eta_{s} \cos \left[D_{s} \theta_{i}(\tau)+D_{s} \psi_{i}(\tau)\right]
\end{aligned}
$$

with $\eta_{s}$ the dipole fugacity for a dipole with separation $s a$ between its positive and negative QPS; we will refer to $s$, loosely, as the moment of the dipole; $D_{s}$ is the difference operator of distance $s: D_{s} \theta_{i} \equiv \theta_{i+s}-\theta_{i}$.

The argument of the cosine function in Eq. (51) places two phase slips of opposite signs on junctions a distance $s a$ apart. Otherwise, this term is completely analogous to the cosine term in Eq. (48). Note that the dipole term resembles the Josephson term in the original action for the JJ array (12), but it is written in the dual variables. The similarity is explained by the following description of the effect of the two terms: $\cos \left(\phi_{i}-\phi_{j}\right)$ removes a Cooper-pair at site $j$, and creates a Cooper pair at site $i$. Similarly, $\cos \left\{\left[\theta_{i}(\tau)+\psi_{i}(\tau)\right]\right.$ $\left.-\left[\theta_{j}(\tau)+\psi_{j}(\tau)\right]\right\}$, creates a phase-slip on bond $i$, and an antiphase-slip on bond $j$.

Initially, there are no phase slip dipoles, and $\eta_{s}=0$ for all $s$. However, upon coarse graining in time, dipoles will form and have to be analyzed as independent entities, on the same footing as single phase slips.

\section{Phases of the JJ array}

The three phases of the JJ array can be described in terms of phase slips. The simplest is the normal phase (NOR), in which phase slips proliferate, and phase coherence is lost. In the $\mathrm{SC}^{\star}$ phase, phase-slips and anti-phase-slips bind into (neutral) dipoles and the chain becomes globally superconducting although the phase fluctuations on each grain are large enough that the local phase is no longer well defined and the dipoles still proliferate. The phase fluctuations are strong enough that there is no quasi-long-range order of the phase. Nevertheless uniform supercurrent can flow unimpeded in the chain.

In the fully superconducting phase (FSC), the phase slip dipoles bind into quadrupoles and annihilate at low energy scales: the phase differences on each junctions are then well defined, there is quasi long-range order, and the chain is locally, as well as globally, superconducting. We first study these phases and the phase transitions between them via a renormalization group analysis of the sine-Gordon representation of the QPS action.

The two superconducting phases were discussed before. ${ }^{42,43,45,46}$ The phase diagram of the RSJJ chain for $r$ $=0$ was discussed by Chakravarty et al. ${ }^{28}$ using the selfconsistent harmonic approximation to treat the model of Eq. (12). While this analysis is appealing due to its simplicity, it misses an important distinction between the two distinct superconducting phases. A contemporary publication by Korshunov, ${ }^{42,43}$ analyzed the Dyson equation of the sine- 
Gordon representation Eq. (48) and found the two superconducting phases, dubbing them SC-1 and SC-2. Unfortunately, this approach was not very transparent and does not give a simple physical picture of the superconductor-to-normal transition. Fazio et al. ${ }^{46}$ obtained a similar phase diagram to Korshunov via numerical calculations. In all these papers only the $r=0$ case was considered. In this paper we analyze the model of Eq. (12) for general $r$ and provide further insight into the nature of the superconductor-to-normal transitions. For the case $r=0$, we reproduce a phase diagram derived by Korshunov in Refs. 42 and 43. We should emphasize that our RG analysis is the first to allow an investigation of both phase boundaries and critical phenomena in the entire phase diagram (with the exception of intermediate Josephson strength, where, to date, no analytical method applies). Having the RG framework allows us to discuss both finite size and finite temperature effects of the dissipative JJ chain.

\section{Renormalization group flows for strong Josephson coupling}

As we showed above, in the limit of strong Josephson coupling the RSJJ chain of Fig. 1(c) can be described by a spatially discrete sine-Gordon model

$$
Z=\int D[\theta] D[\psi] e^{-S_{\text {dual }}[\theta, \psi]-S_{\text {dipole }}[\theta, \psi]} .
$$

We now derive and analyze the RG flow equations in this strong Josephson coupling limit, treating the QPS fugacities as small, but at the same time allowing any values of the shunt and conversion resistances.

The flows of the anisotropic RG we use, are produced in the sine-Gordon representation by integrating over high frequency modes $\theta^{>}$and $\psi$ in a frequency shell $\Omega-d \Omega$ $<|\omega|<\Omega$ of $\theta_{i}(\tau)$ and $\psi_{i}(\tau)$, and then rescaling the time as $\tau \rightarrow \tau^{\prime}=\left(1-\frac{d \Omega}{\Omega}\right) \tau$ so that $\Omega$ returns to its original value, and the differential flow parameter is

$$
d l \equiv \frac{d \Omega}{\Omega} .
$$

However, crucially, lengths are not rescaled and the spatial discreteness is kept. In doing this, however, there appears a characteristic length scale $\Lambda(\Omega)$ that plays an important role because of the anisotropic RG procedure: in terms of the (renormalized) phase velocity $c$ which grows under renormalization because of the rescaling of time this is

$$
\Lambda=\frac{c}{a \Omega} .
$$

Appendix 5.B of Ref. 82 describes in detail the derivation of the RG-flow equations perturbatively in powers of the fugacities; we will need these up to second order.

Integrating out the fast modes, effectively increases the short time cutoff scale from $\tau_{0}$ to $\tau_{0}+d \tau_{0}=\tau_{0}(1+d \Omega / \Omega)$ so that part of the phase slip action is absorbed into its core yielding a linear suppression of the phase-slip fugacities. However, there is also an increase in the fugacities from rescaling time as they represent the rate of phase slips. Combining these two effects we get

$$
\begin{gathered}
\zeta \rightarrow \zeta\left[1+\frac{d \Omega}{\Omega}\left(1-\frac{1}{2} \sqrt{K}-\alpha\right)\right], \\
\eta_{s} \rightarrow \eta_{s}\left\{1+\frac{d \Omega}{\Omega}\left[1-2 \alpha\left(1-w^{s}\right)-K\left(1-e^{-s a / \Lambda}\right)\right]\right\},
\end{gathered}
$$

where $K=\sqrt{\kappa J}$.

The effective dissipation for the $s$ dipoles is

$$
2 \alpha\left(1-w^{s}\right),
$$

which can be seen to be the inverse of the shunt resistance for the pair of (here parallel) Josephson junctions that couple a row of $s$ grains to the rest of the chain, as derived in Sec. II B. As it is necessary to treat the smallest dipoles $s=1$, especially, we define

$$
\beta \equiv 2 \alpha(1-w) .
$$

The $K$ dependent term in the renormalization of $\eta_{s}$ requires some explanation. For dipoles with large $s$, the two constituent QPS will be separately renormalized by the phase fluctuations that have been integrated out: thus the factor proportional to $K$ in this limit. In terms of the circuit analysis, this dissipation can be thought of as arising from the frequency $\Omega$ plasmons that propagate from the two opposite phase slips before annihilating far away. In contrast, dipoles with small $s$ will couple only weakly to the low energy phase modes and thus $K$ has little effect on their renormalization. The energydependent length scale $\Lambda$ separates these two regimes.

In principle, there should also be energy-scale dependence of the renormalization of $\zeta$, but this can be ignored as long as $\Lambda$ is substantially larger than $a$. We will assume this henceforth at the cost of ignoring only initial transient renormalizations (which will in any case only be part of the other relatively high energy scale processes we have ignored).

Nonlinear contributions to the renormalization of $\zeta$ and the $\left\{\eta_{s}\right\}$ come from second order processes. One such process is the combining of two phase slips of opposite signs into a dipole. The two phase slips have to occur on at almost the same time (within $\tau_{0}$ of each other) on Josephson junctions separated by $s$ grains. This process gives a renormalization of $\eta_{s}$

$$
\eta_{s} \rightarrow \eta_{s}+\zeta^{2} \frac{d \Omega}{\Omega} \tau_{0}\left(2 \alpha w^{s}+K e^{-s a / \Lambda}\right)
$$

again note the $s$ dependence of the $K$ term: the dipoles are only formed with appreciable amplitude when the spacing between them is less than $\Lambda$; for convenience we define the factor

$$
u(l) \equiv e^{-a / \Lambda}
$$

by analogy with $w$.

A dipole can combine with a phase-slip on one of the junctions of the dipole to form a phase slip on the other junction. This adds second order contributions to $\zeta$, for each $s$ : 


$$
\zeta \rightarrow \zeta+\zeta \eta_{s} \frac{d \Omega}{\Omega} \tau_{0}\left[2 \alpha\left(1-w^{s}\right)+K\left(1-u^{s}\right)\right]
$$

Two overlapping dipoles can combine to make a single dipole with moment the sum of the constituent moments. This yields

$$
\begin{aligned}
\eta_{s} \rightarrow & \eta_{s}+\frac{d \Omega}{\Omega} \frac{1}{2} \sum_{\sigma} \eta_{|\sigma|} \eta_{|s-\sigma|}\left[2 \alpha\left(1+w^{s}-w^{|\sigma|}-w^{|s-\sigma|}\right)\right. \\
& \left.+K\left(1+u^{s}-u^{|\sigma|}-u^{|s-\sigma|}\right)\right]
\end{aligned}
$$

where the sum runs over all $\sigma$ to take into the account both relative orientations of the dipoles.

Another important contribution comes from combining two phase slips of opposite signs on the same pair of Josephson junctions with a small delay between them $[1 / \Delta \tau$ $>\Omega(1-d \Omega / \Omega)]$. Their polarization gives rise to a renormalization of the parameter $J$, which is related to the superconducting stiffness of the Josephson junctions. This yields a contribution to the quadratic action

$$
\frac{1}{4} \tau_{0}^{2} \zeta^{2} \sum_{i} \int d \tau \frac{d \Omega}{\Omega} \tau_{0}^{2} \sqrt{J \kappa}\left(\frac{\partial \theta_{i}(\tau)}{\partial \tau}\right)^{2} .
$$

In addition, the rescaling of imaginary time-but not space-leads to a rescaling of the parameters $\kappa \rightarrow \kappa(1$ $-d \Omega / \Omega)$ and $J \rightarrow J(1+d \Omega / \Omega)$ which is reflected in the renormalization of the velocity $c$ and hence $\Lambda \propto c$; at linear order in the fugacities $K=\sqrt{\kappa J}$ is not renormalized.

The differential RG flow equations for $J, \zeta$, and $\left\{\eta_{s}\right\}$ can now be obtained by combining the terms from Eqs. (55), (58), and (60)-(62)

$$
\begin{gathered}
\frac{d J}{d l}=J-\frac{\pi}{2} J^{5 / 2} \kappa^{1 / 2} \zeta^{2} \tau_{0}^{2}, \\
\frac{d \kappa}{d l}=-\kappa, \\
\frac{d \zeta}{d l}=\zeta\left(1-\frac{1}{2} \sqrt{\kappa J}-\alpha\right)+2 \zeta \sum_{s>0} \eta_{s} \tau_{0} \alpha\left(1-w^{s}\right), \\
\frac{d \eta_{s}}{d l}=\eta_{s}\left[1-2 \alpha\left(1-w^{s}\right)\right]+\zeta^{2} \tau_{0}\left[2 \alpha w^{s}+\sqrt{\kappa J}\right] \\
+\frac{1}{2} \sum_{|\sigma|} \eta_{\sigma} \eta_{|s-\sigma|} 2 \alpha\left(1+w^{s}-w^{|\sigma|}-w^{|s-\sigma|}\right),
\end{gathered}
$$

valid in the limit of small fugacities $\zeta$ and $\eta_{s}$ and for low energy scales. As we shall see, for some purposes these flow equations are not sufficient even in this limit: the dependence of the renormalizations of and by dipoles on their moments relative to $\Lambda(\ell)$ - whose renormalization is determined since $\Lambda \propto \sqrt{J / \kappa}$ - need to be included even though, formally, these disappear in the low energy limit of interest. For other purposes, we need to consider the case of large $\left\{\eta_{s}\right\}$ but small $\zeta$.

\section{PHASE DIAGRAM AND PHASE TRANSITIONS FOR STRONG JOSEPHSON COUPLING}

Equation (48), the action for phase slips, allows a complete description of the phase diagram in the strong coupling limit and controlled expansions in the vicinity of the phase transitions for some ranges of parameters. When the fugacity of individual QPS $\zeta$ is relevant, the superconductivity should break down completely and the chain exhibit normal behavior. As $\zeta$ grows, it will also induce phase slip dipoles. If $\zeta$ is irrelevant, on the other hand, the chain should-at least naively - be superconducting, but which superconducting phase obtains depends on the behavior of the dipoles.

The simplest phase in the strong coupling limit is the fully superconducting phase (FSC). In this phase, the fugacities $\left\{\eta_{s}\right\}$ and $\zeta$ are all irrelevant about the Gaussian fixed line of the sine-Gordon model (48), which thus controls the low energy behavior of the FSC phase. Not only will phase slips occur only in tightly bound pairs that will not be apparent at low energy scales, but phase slip dipoles will bind into quadrupoles, and isolated dipoles will not be evident at low energies.

When both $\zeta$ and the $\eta_{1}$-the smallest dipoles that are least costly - are relevant, they will grow, inducing the other $\left\{\eta_{s}\right\}$ to grow as well, until the small fugacity expansion breaks down. Below this scale, there will be free QPS's and no superconductivity: the resulting normal phase is best studied from the weak coupling limit as we do in the next section.

The behavior when $\eta_{1}$ is relevant, but $\zeta$ is not, is more subtle: either the $\mathrm{SC}^{\star}$ phase or the normal phase can be obtained. If the phase slips still bind into dipoles, but the dipoles do not bind into quadrupoles, the $\mathrm{SC}^{\star}$ phase can be obtained. In this phase we can ignore the ohmic dissipation and therefore also the dipoles as separate objects: the proliferated dipoles essentially render the conversion resistance $r$ infinite. Once dipoles proliferate, the $\mathrm{SC}^{\star}$ phase may still be unstable to single QPS. An instability to $\zeta$ once dipoles are proliferated drives the $\mathrm{SC}^{\star}$ phase to the NOR phase, but this stability must be considered differently.

The qualitative discussion above gives rise to the main features of the phase diagram of Fig. 7: the exception being the weak-coupling section of the mixed transition that occurs for small $K$ and small $R$; this we discuss in the next section.

The relevance of $\zeta$ or $\eta_{1}$ about the FSC limit triggers the phase transitions from this phase to the normal and $\mathrm{SC}^{\star}$ phases as well as controlling the bicritical point at which the mixed phase transition changes character. In the limit of very strong coupling, the locations of the phase boundaries are given by the vanishing of the linear eigenvalues of $\zeta$ and $\eta_{1}$.

The $\mathrm{SC}^{\star}$-normal phase boundary can be understood straightforwardly from the $\mathrm{SC}^{\star}$ phase (in which dipoles have proliferated) purely in terms of individual QPS. This transition is triggered by relevance of $\zeta$. Its location, however, cannot be precisely obtained in terms of the original parameters, even in the limit of strong coupling, because of the effects of the proliferation of dipoles.

\section{A. FSC-SC ${ }^{\star}$ phase transition [line $(L)$ ]}

In both the $\mathrm{FSC}$ and $\mathrm{SC}^{\star}$ phases single phase slips are irrelevant. Therefore, to determine the transition between the 

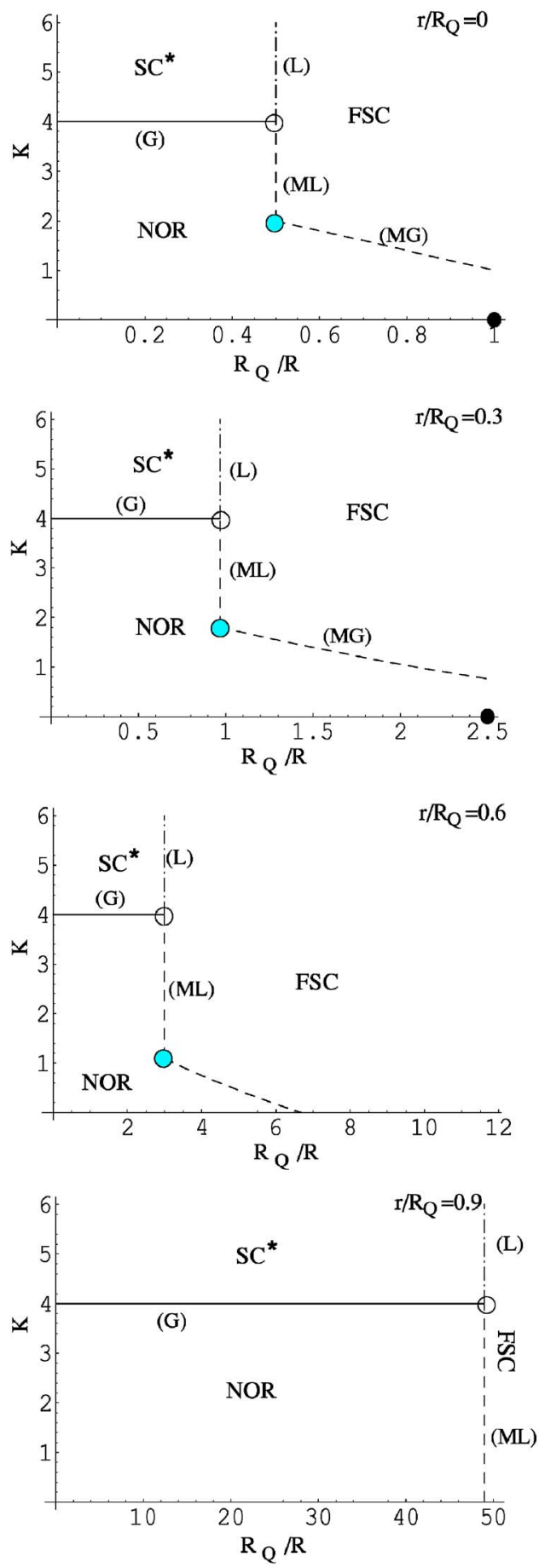
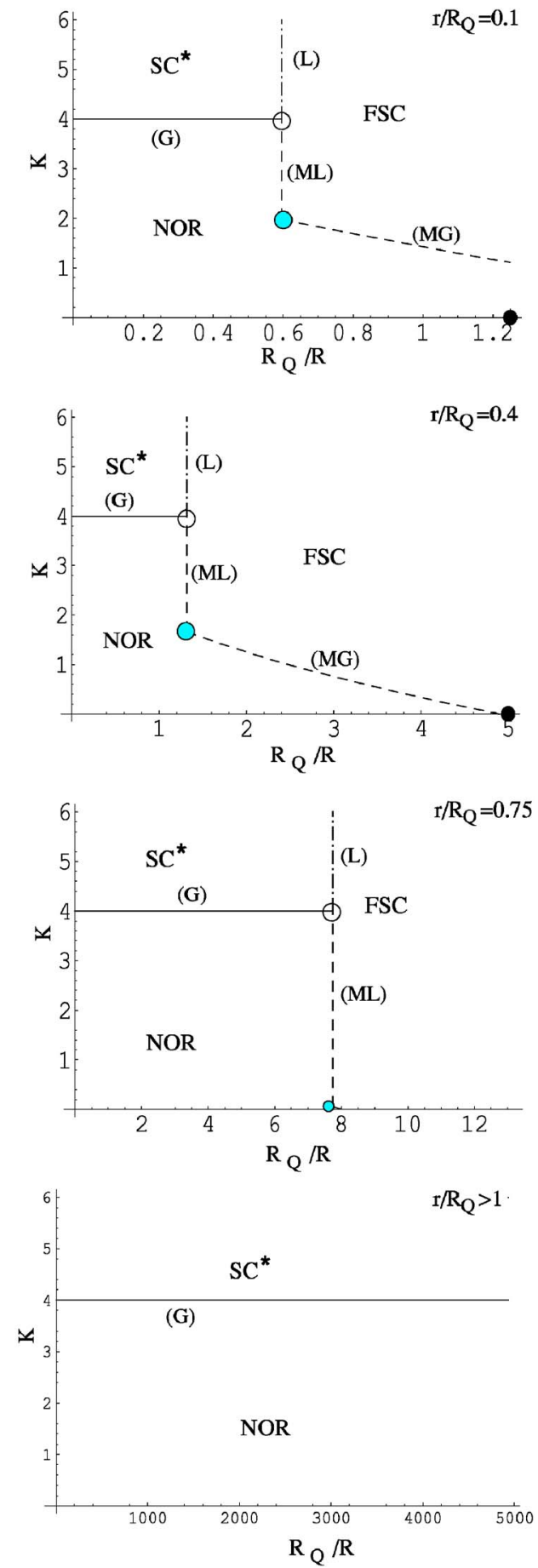

FIG. 7. (Color online) Strong coupling phase diagram in the $K$ $-R_{Q} / R$ plane for various values of $r$. The three phase boundaries between the FSC, $\mathrm{SC}^{\star}$, and NOR can be local, global or mixed. They are marked as following: (G) marks global (solid line), (L) local (dashed-dotted line), and (M) marks mixed (dashed lines). The FSC-NOR transition is partially local (ML) and global (MG) as marked in the figure. The meeting point where the FSC-NOR boundary changes its nature is a bicritical point marked by the gray dot. The open dot Marks the meeting of the three phase boundaries, and is a multicritical point. Black dots mark where superconductivity breaks down in the limit of $K \rightarrow 0$. Note that the phase diagram in the vicinity of the sharp corners (bicritical points) may not be exactly correct due to large renormalizations of $K$ which we otherwise neglect. These regions may give rise to quite complicated multicritical behavior. The critical behavior in the vicinity of the bicritical and multicritical points is richer than near the other phase boundaries, and is characterized by very long crossovers.
FSC and the $\mathrm{SC}^{\star}$ phase we need to know when $\eta_{1}$ (the least costly dipole) changes from irrelevant to relevant about the Gaussian fixed line. The primary role of the non-linear RG flows for these purposes, is the generation of $\left\{\eta_{s}\right\}$ from $\zeta^{2}$ in Eq. (63) so that these will appear and must be considered even when they are absent initially. (Note, however, that the scale at which $\eta_{1}$ becomes important is determined by the $\zeta^{2}$ term so these second order terms can be crucial for understanding finite-temperature and other crossovers.)

The first order terms of Eq. (63) for the flow of $\eta_{1}$ :

$$
\frac{d \eta_{1}}{d l} \approx \eta_{1}(1-\beta)
$$

control the behavior near this transition. In the limit of infinite coupling they yield a boundary line between the FSC and the $\mathrm{SC}^{\star}$ phases

$$
\beta_{L}^{\infty}=2 \alpha(1-w)=1,
$$

which is thus a condition on the critical resistances, e.g., $R_{L}^{\infty}(r)$ for this local transition. The quantitative superfluid 
properties drop out as is the case for individual junctions. As we shall see, for strong but finite coupling, the situation with a chain of junctions is more complicated.

When $\eta_{1}$ is irrelevant about the Gaussian fixed manifold, one must investigate whether there is a critical value of the fugacities above which $\eta_{1}$ grows. For $\beta$ close to unity, an expansion in the fugacities is still possible. The crucial terms are the creation of $\eta_{2}$ by $\eta_{1}$ and the feedback of $\eta_{2}$ on $\eta_{1}$. The structure is

$$
\begin{gathered}
\frac{d \eta_{1}}{d l} \approx \eta_{1}(1-\beta)+C_{112} \eta_{1} \eta_{2}, \\
\frac{d \eta_{2}}{d l} \approx \eta_{2}\left(1-2 \alpha\left[1-w^{2}\right]\right)+C_{211} \eta_{1}^{2}
\end{gathered}
$$

with the $C$ s coefficients. Since the (linear) eigenvalue of $\eta_{2}$ is negative, there is a critical fixed point for $\beta>1$ at

$$
\eta_{1}^{*} \sim \sqrt{\beta-1} \text { and } \eta_{2}^{*} \sim \beta-1 .
$$

It can be seen that the higher moment dipoles have corresponding fixed point values

$$
\eta_{s}^{*} \sim(\beta-1)^{s / 2}
$$

and can thus be neglected to leading order in $\beta-1$. Since $\eta_{1}$ will be of order the bare $\zeta^{2}$ after the brief initial transients, we see that for $R_{L}^{\infty}-R$ small but positive, there will be a critical value of $\zeta$ for the local transition

$$
\zeta_{L} \sim\left(R_{L}^{\infty}-R\right)^{1 / 4}
$$

using, for convenience, $R$ as the control parameter; with $r$ varying instead, the behavior is similar only with a different coefficient.

Just above the critical fugacity, the crossover energy scale below which the chain will no longer appear critical goes to zero near the critical point as

$$
E_{\times} \sim\left|\zeta-\zeta_{L}\right|^{\mu} .
$$

The critical exponent $\mu$ varies continuously with the resistive parameters. For $\beta-1$ small,

$$
\mu \approx \frac{1}{2(\beta-1)} .
$$

Because this transition is essentially local-with dynamic scaling exponent $z=\infty$ - the diverging length scales can be subtle. But there should be some crossover in the spatial correlations at a length scale

$$
\xi \sim c / E_{\times}
$$

beyond which the superconducting correlations behave somewhat differently.

For this discussion, we have not been careful about where the RG flows go when the dipoles proliferate. If they go to the $\mathrm{SC}^{\star}$ phase, the above obtains. However, it is also possible that they go near the $\mathrm{SC}^{\star}$ regime only to flow away at lower energies if $\zeta$ is relevant. In this latter case, the transition will be from the FSC directly to the normal phase, as we discuss below. But we first consider the effects of dipole proliferation.

\section{B. $\mathrm{SC}^{\star}$ phase and screening of dissipative interactions}

In the region of parameter space where $\eta_{1}$ is relevant, the simple perturbative RG flow, Eqs. (63), breaks down, and phase-slip dipoles proliferate. But this is not in itself sufficient to destroy superconductivity; the lead-to-lead coherence can persist as long as the quantum phase slips remain bound in pairs. ${ }^{59,86}$ Dipoles do, however, allow the phase of a single grain to jump by $2 \pi$ relative to its neighbors. This is the $\mathrm{SC}^{\star}$ in which each grain no longer has a well defined phase. But the global superconductivity survives as long as $\zeta$ is irrelevant about the fixed line that controls the $\mathrm{SC}^{\star}$ phase.

In the $\mathrm{SC}^{\star}$ phase, the dissipation no longer plays an essential role. This can be most easily seen by comparing the dipole term with the quadratic parts of the action for the $\psi$ field in the Sine-Gordon action. At low energies, the fluctuations of the field $\theta$ are small enough that one can ignore the $\theta_{j+1}-\theta_{j}$ in the dipole term and approximate it by

$$
\eta_{1} \cos \left[\psi_{j+1}-\psi_{j}\right]
$$

When $\eta_{1}$ becomes of order $\Omega$, the highest remaining frequency, the dipole term is comparable to the $R|\omega|\left|\psi_{j}(\omega)\right|^{2}$ and the $r|\omega|\left|\psi_{j+1}(\omega)-\psi_{j}(\omega)\right|^{2}$ term at frequencies of order $\Omega$. (If $r \ll R$ the comparison is somewhat more subtle.)

Below this crossover frequency, the dipole term strongly suppresses fluctuations of neighboring $\psi_{j}$ at low frequencies: together with the $R|\omega|\left|\psi_{j}(\omega)\right|^{2}$ term, it cuts off the divergent fluctuations of $\psi$ while $\theta$ continues to fluctuate similarly to in the absence of the dipoles. At this point, we can take the continuum limit without worry, replace the lattice with a high-momentum cutoff and expand out the dipole terms (analogous to treating screening in a metal as if by continuous charges - the Debye-Hückel approximation) as

$$
\begin{array}{r}
\eta\left\{\cos \left[a\left(\frac{\partial \psi(x, \tau)}{\partial x}+\frac{\partial \theta(x, \tau)}{\partial x}\right)\right]-1\right\} \\
\approx-\frac{\eta}{2}\left[a\left(\frac{\partial \psi(x, \tau)}{\partial x}+\frac{\partial \theta(x, \tau)}{\partial x}\right)\right]^{2} .
\end{array}
$$

An approximate action for the $\mathrm{SC}^{\star}$ phase is then

$$
\begin{aligned}
S= & \int \frac{d \omega}{2 \pi} \int \frac{d k}{2 \pi}\left\{\left[k^{2}\left(\frac{1}{2 \pi \kappa}+a^{2} \eta\right)+\frac{\omega^{2}}{2 \pi J}\right] \theta^{2}\right. \\
& \left.+\left[a^{2} \eta k^{2}+\frac{\lambda_{Q}}{2 \pi \alpha}|\omega|\left(k^{2}+\frac{1}{\lambda_{Q}^{2}}\right)\right] \psi^{2}\right\} \\
& -\sum_{i} \int d \tau \zeta \cos \left[\theta_{i}(\tau)+\psi_{i}(\tau)\right],
\end{aligned}
$$

where we have neglected $\theta-\psi$ cross-terms proportional to $k^{2}$. These do not contribute to or alter the singular interaction between phase slips in this regime. Note that the plasmon part of the interaction (the $\theta^{2}$ part) has been renormalized by the phase-slip dipoles, but it is not screened, i.e., it still vanishes at $\omega=k=0$. The dissipative part, however, does get screened reducing the dissipative interaction between phase slips to $S_{\text {diss }}(x, \tau) \propto 1 / \tau^{2}$ when $|\tau| \gg \frac{\lambda_{Q}}{4 \pi \alpha \eta a}$. This should be compared with Eq. (42). Because of the finite screening time of the dissipative interaction between phase slips, this does 
not play a role for the asymptotic behavior of either the $\mathrm{SC}^{\star}$ phase, or the $\mathrm{SC}^{\star}$ to normal transition. The screening of the dissipative interaction by dipoles can be understood simply by circuit methods (Sec. II B) or by considering the phase slips as a Coulomb gas. It plays a crucial role for finite JJ chains for which the effects of dissipation are not completely eliminated. This will be discussed in a future publication. ${ }^{71}$

If the system flows to the $\mathrm{SC}^{\star}$ fixed line when the dipole fugacity grows, then the critical behavior of the FSC-SC ${ }^{\star}$ transition is that found in the previous subsection. Before returning to the trickier question of what happens when the flows eventually go away from the $\mathrm{SC}^{\star}$ regime, we briefly discuss the direct $\mathrm{SC}^{\star}$-NOR transition.

\section{C. $\mathrm{SC}^{\star}$-normal transition [line $(G)$ ]}

Once dissipation has been eliminated, we are left with a simple low-energy effective action for the $\mathrm{SC}^{\star}$ phase: the standard chain of grains with self capacitance and connected by Josephson junctions. In the sine-Gordon representation, we have

$S_{\mathrm{SC}^{*}} \approx \int \frac{d \omega}{2 \pi} \int \frac{d k}{2 \pi}\left(\frac{k^{2}}{2 \pi \widetilde{\kappa}}+\frac{\omega^{2}}{2 \pi \widetilde{J}}\right) \theta^{2}-\sum_{i} \int d \tau \zeta \cos \left[\theta_{i}(\tau)\right]$,

where the tildes over the couplings are a reminder that they include renormalizations from integrating out the effects of the dipoles as in Eq. (76).

The action Eq. (77) is most naturally studied by an isotropic RG rescaling both $x$ and $\tau$, and integrating out the modes of $\theta$ that have large $\omega$ or large $k$. By carrying out the RG flow in $x$ and $\tau$ directions, the usual KT RG equations for $K=\sqrt{\widetilde{\kappa} \widetilde{J}}$ and $\zeta$ obtain $^{87}$

$$
\begin{aligned}
& \frac{d K}{d l}=-\frac{\pi}{2} K^{2} \tau_{0}^{2} \zeta^{2}, \\
& \frac{d \zeta}{d l}=\zeta\left(2-\frac{K}{2}\right) .
\end{aligned}
$$

The $\mathrm{SC}^{\star}$-normal phase boundary-a global transition-thus occurs for $\zeta \ll 1$ at

$$
K_{G}=4+\mathcal{O}(\zeta) .
$$

When the initial $\zeta$ is not small, the critical value of $K$ will be increased both by the direct effects of $\zeta$ and also via its generation of dipoles $\left(\left\{\eta_{s}\right\}\right)$ and their later modification of $\kappa$ at intermediate scales as in Eq. (76).

In the normal phase near this global transition, the characteristic time and length scales both diverge exponentially as $\exp \left[1 / \sqrt{K_{G}-K}\right]$ - and similarly in $R-R_{G}$ when dissipation plays a role at intermediate scales and can control the transition. This contrasts with the power law divergences near the FSC-SC ${ }^{\star}$ local transition.

Although we have here used the conventional isotropic $\mathrm{RG}$ to study the $\mathrm{SC}^{\star}$-normal transition, it is important to understand whether the anisotropic RG we use in the rest of this paper can reproduce the KT results in the appropriate regime. We address this issue below in Sec. IV $\mathrm{H}$.

\section{FSC - Normal transition [line $(M)$ ]}

We now turn to the most subtle transition: the mixedcharacter transition from the FSC to the normal phase. As the phase slip fugacity is increased, the superconducting stiffness $K$ is reduced, or the resistances increased, a quantum phase transition between the FSC phase and the normal phase of the chain can occur directly without an intermediate $\mathrm{SC}^{\star}$ phase. This mixed transition can be driven by two distinct mechanisms even in the strong coupling limit in which the Sine-Gordon description is useful. If single QPS's become relevant when dipoles are still irrelevant about the FSC fixed line, they will drive the transition inducing proliferation of both dipoles and isolated QPS as the fugacity $\zeta$ grows.

However, if small dipoles become relevant when single QPS are still irrelevant about the FSC fixed manifold, it is nevertheless still possible for the dipole-driven transition to be between the FSC and normal phases. This occurs if the flow towards the $\mathrm{SC}^{\star}$ phase from the proliferating dipoles is interrupted by the relevance of individual QPS about the $\mathrm{SC}^{\star}$ fixed line. We first discuss the latter case.

\section{E. Dipole driven mixed transition [line $(M L)$ ]}

The RG flows for this dipole driven transition are somewhat complicated. As shown earlier, if the QPS fugacity is irrelevant about the FSC fixed manifold when the dipole fugacities start growing - such as when the bare fugacity $\zeta$ is above a critical value $-\zeta$ will continue to decrease until the effects of the dipoles are strong enough to change its flow. Crudely, this will happen when the $\eta_{s} \zeta$ terms in Eq. (63) become large enough to dominate over the $\left(\frac{1}{2} K+\alpha-1\right) \zeta$ term. As this will happen when some of the $\left\{\eta_{s}\right\}$ are of order the energy scale $\Omega$, say at energy scale $E_{\times}$, the system will soon after approach the $\mathrm{SC}^{\star}$ fixed line as discussed in Sec. IV A (other operators neglected perturbatively will also become important on these scales). However, by this point, if the system is close enough to critical, $\zeta$ will have become extremely small. Thus even when it is relevant about the $\mathrm{SC}^{\star}$ fixed line and hence will turn around are grow on scales smaller than $E_{\times}, \zeta$ will not become large enough to make individual QPS proliferate, until a much lower energy scale $E_{N}<E_{\times}$.

Physical properties should exhibit both of the energy scales just beyond the FSC-normal transition: At high energies, the system will appear critical-FSC-SC ${ }^{\star}$ critical-at intermediate energies $E_{N} \ll E \ll E_{\times}$, it will appear to be in the $\mathrm{SC}^{\star}$ phase-albeit with a phase stiffness that is too low to sustain superconductivity at long scales-and at asymptotically low energies $E \ll E_{N}$ normal behavior will obtain. This behavior is characteristic of a dangerously irrelevant operator-in this case $\zeta$-about the critical fixed point. The energy scale $E_{N}$ will go to zero as a power of the distance from criticality, but its exponent will be larger than that of $E_{\times}$by an amount controlled by both the flow of $\zeta$ towards the FSC fixed manifold, and its flow away from the $\mathrm{SC}^{\star}$ fixed line, as well as the flow of $\eta_{1}$ away from the critical fixed point. The behavior of the length scales will be simi- 
larly complicated and the dynamic critical exponent relating $E_{N}$ to the superconducting correlation length, will be larger than unity.

\section{F. QPS driven mixed transition [line $(M G)]$}

In the regime in which the FSC-normal transition is driven by QPS - the fundamental topological excitationsthe phase boundary and nature of the transition can be studied from Eqs. (63) for the flow of $\zeta$. For this transition, the dipoles play only a secondary role.

In the region near the FSC fixed manifold, the perturbative RG equations (63) are valid and, in the regime of interest, the $\left\{\eta_{s}\right\}$ are renormalized to zero and can be ignored. We thus need only the first two RG flow Eqs. (63). Working in units of the cutoff energy scale $\Omega$ and length scale $a$, we have

$$
\begin{gathered}
\frac{d J}{d l} \approx J-\frac{\pi}{2} J^{5 / 2} \kappa^{1 / 2} \tau_{0}^{2} \zeta^{2}, \\
\frac{d \kappa}{d l} \approx-\kappa, \\
\frac{d \zeta}{d l} \approx \zeta\left(1-\frac{1}{2} \sqrt{\kappa J}-\alpha\right) .
\end{gathered}
$$

Equations (80) are similar to the Kosterlitz-Thouless (KT) flow equations, except for the anisotropic scaling of $\kappa$ and $J$. We first proceed naively and transform to variables in which the flows appear more isotropic, defining

$$
\begin{gathered}
\bar{\kappa}=\kappa e^{l}, \\
\bar{J}=J e^{-l}, \\
\bar{\zeta}=\zeta e^{(1 / 2) l} .
\end{gathered}
$$

whereby Eqs. (80) become

$$
\begin{gathered}
\frac{d \bar{\kappa}}{d l}=0, \\
\frac{d \bar{J}}{d l}=-\frac{\pi}{2} \bar{J}^{5 / 2} \bar{\kappa}^{1 / 2} \bar{\zeta}^{2} \tau_{0}^{2}, \\
\frac{d \bar{\zeta}}{d l}=\bar{\zeta}\left(\frac{3}{2}-\frac{1}{2} \sqrt{\bar{\kappa} \bar{J}}-\alpha\right) .
\end{gathered}
$$

Shifting and rescaling the variables via

$$
\begin{gathered}
x=\frac{\sqrt{\bar{\kappa} J}}{2}+\alpha-\frac{3}{2}, \\
z=\sqrt{\frac{\pi \bar{\kappa}}{8}} \bar{J} \tau_{0} \bar{\zeta} .
\end{gathered}
$$

so that $x$ parametrizes deviations from the special point, the flow Eqs. (82) assume the canonical KT form

$$
\frac{d x}{d l} \approx-z^{2}, \quad \frac{d z}{d l} \approx-z x .
$$

The flows of Eq. (84) suggest a phase boundary between the normal and FSC phases. In the FSC phase $z$ flows to zero whereas in the normal phase $z$ diverges and $K$ flows to zero. The critical line separating the two phases is thus

$$
x_{M} \approx-z \text {. }
$$

When $\zeta \ll 1$ the FSC-normal phase boundary in Eq. (85), $x$ $\approx 0$, translates to

$$
K_{M}=3-2 \alpha .
$$

Although the phase boundary of Eq. (86) is correct for the renormalized $K$, for nonzero initial QPS fugacity $\zeta$, the critical value of $K$ will be slightly larger-by $\mathcal{O}\left(\zeta^{2}\right)$ when the bare fugacity is small-because of the renormalizations of Eq. (82).

On the normal side of the transition, the behavior is subtle. The subtlety arises because the transformation between $\bar{\zeta}$ and $\zeta$ [Eqs. (82) and (83)] involves the scale of the RG explicitly: $\zeta \propto e^{-l / 2} \bar{\zeta} \propto e^{-l / 2} z$, so that $\zeta$ may decrease even though $z$ is formally relevant. However, as $z$ grows to the order unity, it will substantially decrease $x$, concomitantly, $\bar{J}$, and hence the further growth rate of $\bar{\zeta}$ from Eq. (81). The growth rate of $\bar{\zeta}$ will then become rapid enough to overcome the $e^{-l / 2}$ factor and make $\zeta$ grow as well. Thus after initially decreasing because of the factor $e^{-l / 2}, \zeta$ eventually turns around and then becomes large (at not much lower energies) at which point the behavior will be characteristic of the normal phase.

The dipole fugacities $\left\{\eta_{s}\right\}$ will be small as long as $\zeta$ is, but when $\zeta$ becomes large, they will grow (for large $s$ as well as small). This implies that Josephson junctions are no longer independent of each other, and phase slips become spreadout over several junctions. In principle, at this stage we could switch to carrying out the RG isotropically, but by then other higher order processes will also come in and expansions in powers of the fugacities break down.

The critical behavior near this QPS driven mixed transition will be similar to near a conventional KosterlitzThouless transition, with, for example, energy scale $E_{\times}$vanishing exponentially in the inverse square root of the distance from criticality. The corresponding exponent is $\mu=\infty$ in contrast to the finite but variable $\mu$ that characterizes the dipole driven mixed transition. Associated with the $E_{\times}$will be one or more diverging length scales. There should be a scale that grows as $1 / E_{\times}$corresponding to dynamical exponent $z=1$, but there may be a second scale associated with the onset of substantial screening of $\kappa$ and spatially (rather than temporally) separated QPS that will occur only after the dipoles come into play. We will not explore this issue further here.

To make the above analysis solid, It is essential to show that the perturbative RG equations on which it is based are valid out to scales at which $K(\ell)$ starts to decrease significantly from the screening by pairs of QPS. The rescaling from $\zeta$ to $\bar{\zeta}$ was chosen to make these renormalizations, as in Eq. (82), small if and only if $\bar{\zeta}$ remains small. This could better (but equivalently to the needed order) have been done 
by defining instead $\widetilde{\zeta}=\sqrt{J} \zeta$ thereby making $d \ln J / d \ell$ depend only on the combinations $\widetilde{\zeta}$ and $K=\sqrt{J \kappa}$ : the latter (in contrast to, e.g. $c=\sqrt{J / \kappa}$ ) is of order unity in the regime of interest near the transition. With this choice, it means that the dipole fugacities $\left\{\eta_{s}\right\}$ are induced by $(\widetilde{\zeta})^{2} / J$ which decreases rapidly after the initial transients that create small dipoles. Thus the $\left\{\eta_{s}\right\}$ should not be problematic as they will be decreasing and only give rise to relative changes: terms of or$\operatorname{der} \zeta \eta_{s}$

The primary potential problems are additional renormalizations of $J$ (or $\kappa$ ) that are not small when $\tilde{\zeta}$ is small. These can arise, for example, by the combination of a tripole-a QPS “dressed" by a nearby small dipole - and an opposite sign QPS. Such terms are equivalent to terms of order $\widetilde{\zeta}^{4}$ in other RG schemes, but these, and other terms higher order than quadratic, do not occur directly with the RG scheme we use: they are generated from other operators as in this case. This has the advantage of making a cataloging of all operators and how they renormalize each other linearly and quadratically, equivalent to "all orders" arguments in other perturbative RGs. One can readily show that the generation of tripoles from a dipole and a QPS, and the subsequent renormalization of the tripole fugacities by dissipation plus the stiffness $K$, will result in effects of the tripoles back to $\widetilde{\zeta}$ and to $J$ that decay with energy scale while $\tilde{\zeta}$ (and equivalently $z$ ) is decreasing. Further analysis along these lines shows that indeed, the result for the critical $K_{M}$, Eq. (86) is correct. What is rather surprising, is that this arises from a basic (power-counting-like) renormalization of $\zeta$ that is midway between the " 1 " for purely temporal rescaling, and the " 2 " for space-time rescaling.

By showing that the RG remains controlled out to this stage, we have verified that the FSC-normal phase boundary is in one regime indeed given by Eq. (86) in the limit of small fugacities; more generally the location of the transition will be exactly $K=3-2 \alpha$ if the renormalized-and hence measurable-low energy parameters $K$ and $\alpha$ are used. Nevertheless, near the phase boundary on the normal side, the fact that $\zeta$ first flows toward zero and only at a later stage becomes large, gives rise to a crossover in the resistance vs. temperature. From the last of Eqs. (80) we see that this will occur as long as

$$
K>2-2 \alpha .
$$

Thus the crossover occurs in the range of parameters

$$
2-2 \alpha<K<3-2 \alpha
$$

the physical consequences and origin of this region is discussed in Secs. VII and II B.

\section{G. FSC-Normal bicritical point}

As shown above in Secs. IV E and IV F, the FSC-normal transition can have one of two distinct characters: either a dipole driven transition with power-law singularities [line $(M L)$ in Fig. 7], continuously variable exponents, and nontrivial relations between length and time scales, or driven by single QPS, with exponentially rapidly decreasing energy scales and at least some space and time scales scaling similarly [line $(M G)]$. In the limit of small (bare) fugacities, the transition occurs where in the $K, \alpha, \beta$ space the system is at intermediate energy scales. The two critical surfaces in this space meet along a bicritical line, across which the critical behavior changes, although the phases on both sides are the same. At nonzero fugacity $\zeta$ the behavior is similar with the critical surfaces (actually now hypersurfaces) and location of the bicritical line (actually now a two-dimensional manifold) changed. When the critical fugacity is small, as occurs when $\beta-1$ [see Eq. (64)] is small (and positive), the behavior near the bicritical line can be treated perturbatively. If the multicritical manifold is approached along the dipole-driven critical manifold, the former can be found by considering the eigenvalue of $\zeta$ at the point on the critical fixed manifold, parametrized perturbatively by $\eta_{1}^{*}$. This eigenvalue will be increased by $\mathcal{O}\left(\eta_{1}^{*}\right)^{2}$ from its value at the corresponding point on the FSC fixed manifold (all $\left\{\eta_{s}\right\}$ zero). When the critical eigenvalue, rather than $1-\frac{1}{2} \kappa-\alpha$ passes through $-\frac{1}{2}$, the critical fixed manifold will become unstable to individual QPS at low energies. This condition thus characterizes the multicritical manifold. For $\eta_{1}$ just below the bicritical manifold the flow will be to the FSC phase, but because of the initial effects of $\eta_{1}$ on $\zeta$, will end up closer to critical than it would have been. Associated with the multicriticality will be complicated crossover behaviors that we will not explore.

\section{H. SC ${ }^{\star}$-Normal transition revisited}

In the standard treatment of isotropic two-dimensional (2D), or $(1+1) \mathrm{D}, X-Y$ models, we use isotropic RG to obtain the KT transition. This is also the way in which we analyzed the global $(G)$ NOR-SC $^{\star}$ transition in Sec. IV C once dipoles are proliferated. As mentioned earlier, for consistency, we should be able to analyze this global $\mathrm{SC}^{\star}$-normal transition by the anisotropic RG we use in the rest of this paper. This also serves to make a more convincing case for the peculiar behavior of the QPS driven FSC-normal transition. Analyzing the global transition by the anisotropic RG is possible but clumsy; we only outline the basics here.

It is useful to first consider what modification there would be to the small-fugacity flow equations if opposite-sign QPS on different junctions could annihilate each other under renormalization rather than combining into dipoles. Because of factors of $u^{s}=\exp [-a / \Lambda]$-similar to those in the generation of $\eta_{s}$ by $\zeta^{2}$ in Eq. (63) - only QPS pairs separated by less than or of order $\Lambda(\ell)=\sqrt{J / \kappa} / \Omega$ will be renormalized away. However, this is a factor of $\Lambda / a$ more combinations than were included from QPS pairs on the same junction: the renormalization of $J$ of order $\zeta^{2}$ will thus be larger by a similar factor. We now recombine parameters similarly to what was in the mixed case: to make the renormalizations of $J$ small when $\tilde{\zeta}$ is small and $K$ of order unity. This now requires $\widetilde{\zeta}=J \zeta$ (contrast with $\sqrt{J} \zeta$ in the mixed case) which will change the eigenvalue of $\widetilde{\zeta}$ to $2-\frac{1}{2} K-\alpha$ while keeping $K$ marginal. In the absence of dissipation, this would yield a KT transition with critical value $K_{G}=4$ as obtained from the 
isotropic RG of Sec. IV C. What is not immediately clear, is at what point the flows have crossed over into the normal regime and $\zeta \cos \theta_{j}$ can be well approximated by $\zeta\left(1-\frac{1}{2} \theta_{j}^{2}\right)$ and the $\Sigma_{j}$ approximated by $\frac{1}{a} \int d x$. This can be checked by estimating the mean-square fluctuations of $\theta_{j}$ using the resulting quadratic continuum form. As at low energy scales $1 / \kappa$ is large and $1 / J$ small, this will be dominated by wave vectors of order $1 / \Lambda$ or smaller. The remaining frequency integral will be cut off at $\sqrt{\zeta J}$ and thus dominated by frequencies of order $\Omega$ when $\widetilde{\zeta}=\zeta J / \Omega$ is of order unity as would be naively guessed from the rescaling that gave the KT flows.

However, this is not the whole story. In addition to the renormalizations of $J$, there should be similar (asymptotically) renormalizations of $\kappa$ which controls the spatial gradients of $\theta$. In the anisotropic RG, these arise from replacing the dipole operators in the absence of $\psi, \cos \left(\theta_{j+s}-\theta_{j}\right)$, by $1-2\left(\theta_{j+s}-\theta_{j}\right)^{2} \approx 1-2 s^{2} a^{2}\left(\partial_{x} \theta\right)^{2}$. This is justified if $\theta$ is sufficiently slowly varying in space. With the anisotropic cutoff, this is justified for $s<\Lambda$ as, because of the low frequency cutoff relative to the wave-vector dependence $\left\langle\left(\theta_{j+s}-\theta_{j}\right)^{2}\right\rangle$ $\approx 2 K|s| / \Lambda$ in this regime. Thus the renormalizations of $1 / \kappa$ to order $\zeta^{2}$ are of the correct form. By summing over the appropriate range of $s$, their amplitude can be found to be of order $\tilde{\zeta}^{2} \Lambda$ which have the additional $\Lambda$ factor that compensates exactly for the linear growth of $1 / \kappa$ with the inverse of the energy scale in the anisotropic RG. Similarly, the argument in the previous paragraph for how $J$ is renormalized, can be justified by the replacement of $\left(\partial_{\tau}\left[\theta_{s+j}\right.\right.$ $\left.\left.+\theta_{j}\right]\right)^{2} \cos \left(\theta_{j+s}-\theta_{j}\right)$ by $4\left(\partial_{\tau} \theta \frac{1}{2} s+j\right)^{2}$ renormalizing $1 / J$.

For the $\mathrm{SC}^{\star}$-normal transition when the fugacities are small, the analysis above can only be done once the dipoles have been induced and proliferated enough to suppress the fluctuations of $\psi$ and enable it to be neglected as in Sec. IV C. Again, the intermediate regime of the flows, where the $\left\{\eta_{s}\right\}$ are of order unity, cannot be handled in a controlled manner. However, this occurs over a small range of energy scales and thus will only result in factors of order unity.

\section{Multicritical point}

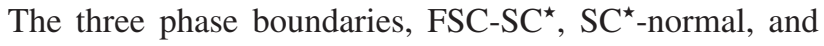
FSC-normal come together at a multicritical point as shown in the schematic phase diagram, Fig. 7. The behavior near this multicritical point involves an interplay between the $\mathrm{SC}^{\star}$-like behavior when dipoles proliferate, and the FSC-like behavior when the dipole fugacities are small. How these come together at the multicritical point is likely to involve flows in the intermediate coupling regime in which controlled calculations are beyond the scope of the methods of this paper.

\section{WEAK JOSEPHSON COUPLING LIMIT}

In this section we analyze the JJ chain when the Josephson interaction is weak: $E_{J} \ll E_{C}$. In this limit the starting point is to assume there is no phase coherence between neighboring grains, so that the currents along the chain are predominantly normal. We can then consider Cooper-pair- tunneling events perturbatively. In this limit the Josephson coupling is sufficiently weak that superconductivity can only be established if the dissipation is sufficiently strong to drive the system into the FSC phase: the $\mathrm{SC}^{\star}$ phase cannot occur as its stiffness $K=2 \pi \sqrt{E_{J} / E_{C}}$ is too small. We thus can explore only the dissipation-dominated transition directly from the normal to the FSC phase: as both local and global superconducting coherence are established by this transition, it has mixed character. As discussed in Ref. 88, pair tunneling events are dual to quantum phase slips. Nevertheless, the treatment is substantially simpler than that of the mixed transition in the strong coupling limit analyzed in the previous section.

\section{A. Pair-tunnel events}

The basic objects that need to be considered are Cooper pair-tunneling events between grains. Their action can be formulated in the Coulomb-gas representation. This allows the RG flows when pair tunneling is rare to be worked out perturbatively.

Consider first the Hamiltonian of a single capacitively shunted Josephson junction

$$
H_{\mathrm{JJ}}=\frac{1}{2 C} \hat{Q}^{2}-E_{J} \cos \phi
$$

In the weak coupling limit, the charge fluctuations will be small relative to the phase fluctuations, and the junction can be described in terms of charge states with wave functions

$$
\Psi(\phi)=e^{i(q / 2 e) \phi},
$$

where $q$ is the charge imbalance across the junction. The cosine term in the Hamiltonian thus induces hopping of Cooper pairs, since $e^{i \phi}$ is a translation operator that changes the charge imbalance by $2 e$. We can describe the normal-tosuperconductor phase transition of a resistively shunted Josephson junction in term of pair-tunnel events: when these are suppressed at low energies, the junction is well described by the charge state (89) and is in the normal state. However, when pair tunnels proliferate, superconductivity is established and the phase becomes the good quantum number. In this dual description, in opposition to that in terms of QPS, the quantum fluctuations are responsible for the superconductivity rather than destroying it.

To explore the pair-tunnel physics, we can start with the action in terms of the superconducting phases $\left\{\phi_{j}\right\}$ of the grains in the chain. This is given by Eq. (12). Since we are interested in the low energy dynamics on small length scales, we take the limit of small $\omega$ of the quadratic parts of the action, keeping the spatial discreteness but dropping the $\mathcal{O}\left(\omega^{2} \phi^{2}\right)$ charging energy. The partition function in this limit can be written in the form 


$$
\begin{aligned}
Z= & \int D\left[\phi_{i}(\tau)\right] \exp \left[-\frac{1}{2} \int \frac{d \omega}{2 \pi} \int \frac{d k}{2 \pi}\right. \\
& \times \frac{|\omega| R_{Q}}{2 \pi a\left[R+r\left(2-e^{i k a}-e^{-i k a}\right)\right]}|\Delta(k, \omega)|^{2} \\
& \left.+\sum_{i} \int d \tau E_{J} \cos \left[\Delta_{i}(\tau)\right]\right]
\end{aligned}
$$

or equivalently with a local part on each grain, and an exponentially decaying - with $w^{s}$-interaction of opposite sign between phases on grains $s$ apart that exactly cancels for the total interaction (i.e., vanishes at $k=0$ ). This is because it is the phase differences across the junctions

$$
\Delta_{i}(\tau)=\phi_{i+1}(\tau)-\phi_{i}(\tau)
$$

that enter here. Note that in the normal phase, which is the starting point here, it would be more proper to write the resistive part of the action in terms of $d \Delta_{j} / d \tau$, which is proportional to the electrochemical potential differences across the junctions as the phase variables are not defined modulo $2 \pi$. At low energies and when the capacitative interactions dominate, the densities of states parameters $D_{N}$ and $D_{S}$ drop out. Note that if there were no normal conduction across the junctions, i.e., $R=0$, the quadratic action would separate into a sum over noninteracting grains.

The role of pair-tunneling events, and hence the superconducting behavior of the JJ chain, can be seen by carrying out an expansion of Eq. (90) in powers of $E_{J}$. The $n$th order term corresponds to the probability weight of $n$ pair tunnels occurring in space time. If the weight of the partition function (90) is concentrated in the term of 0th order in $E_{J}$, it means that no superconductivity is taking place at $T=0$, since pairtunnel events are suppressed. On the other hand, if the weight is concentrated on high order terms, pair-tunnel events proliferate-i.e., they happen frequently-and the chain is superconducting.

The expansion in $E_{J}$ leads to a Coulomb gas representation of the action of pair-tunnel events and the partition function

$$
\begin{aligned}
Z= & \sum_{N}\left(\frac{E_{J}}{\Omega}\right)^{N} \\
& \times \sum_{\{\sigma, x, \tau\}} \exp \left[-1 / 2 \sum_{m=1}^{N} \sum_{n=1}^{N} \sigma_{m} \sigma_{n} G\left(x_{m}-x_{n}, \tau_{m}-\tau_{n}\right)\right],
\end{aligned}
$$

where $\{\sigma= \pm 1\}$ are the "charges"-i.e., signs—of the tunnels, the sum is over all distinguishable neutral configurations with $\sum_{n} \sigma_{n}=0$, and the interaction between tunnel events is

$$
G(x, \tau)=-2 q_{1} q_{2} \ln \left(\frac{|\tau|}{\tau_{0}}\right)\left(\frac{R+2 r}{R_{Q}} \delta_{x, 0}-\frac{r}{R_{Q}}\left(\delta_{x, a}+\delta_{x,-a}\right)\right),
$$

with $\delta_{x_{1}, x_{2}}$ the Kronecker $\delta$. The short time cutoff, $\tau_{0} \sim(R$ $+2 r) C / \hbar$ is now the time duration of a phase slip, essentially
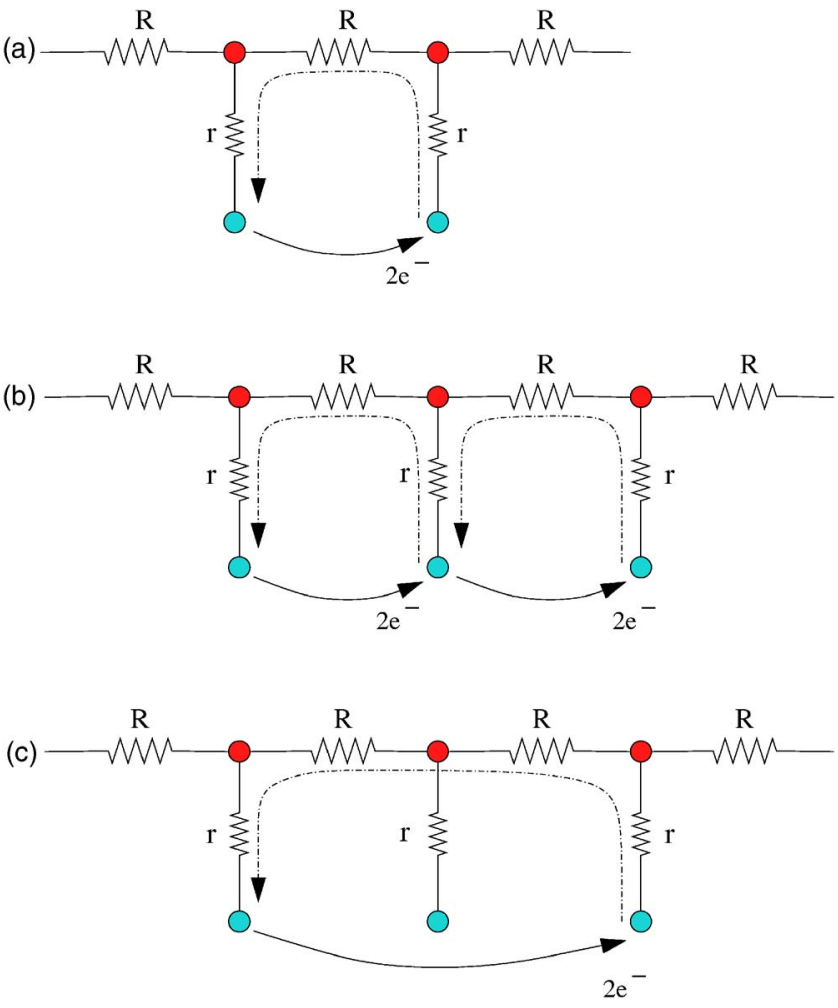

FIG. 8. (Color online) (a) Cooper pair tunneling event, between two neighboring grains (solid line): the basic process for weak Josephson coupling. Charge relaxation is via the resistance $2 r+R$ (dotted line). (b) Two nearly simultaneous pair tunnels involving a common grain: the current through the conversion resistance $r$ of that grain cancels giving rise to an effective interaction between the two pair tunnels. These two pair tunnels can renormalize into a pair tunnel over two junctions (c).

the $\mathrm{RC}$ relaxation time of a charge imbalance between two neighboring grains. This and other cutoffs from the high energy physics, we approximate by a UV cutoff $\Omega_{0}=1 / \tau_{0}$. The tunneling fugacity we denote

$$
\xi_{1} \propto E_{J}
$$

and it has units of frequency.

The distance dependence of the interaction (93) is easy to understand. If two pair-tunnel events happen through the same Josephson junction, the strength of the logarithmic interaction between them is $2 \frac{R+2 r}{R_{O}}$ the total resistance that the Cooper pair has to go through in order to relax back to an equilibrium charge distribution [Fig. 8(a)] as found for a single shunted Josephson junction (see, e.g., Ref. 59). If two pair-tunnels happen on neighboring junctions, the Cooperpair relaxation current overlaps on the common grain and thus involves the normal-to-superfluid resistance within the grain $r$ [Fig. 8(b)]. This reduces the logarithmic interaction strength of $2 \frac{r}{R_{Q}}$ between neighboring tunnel-events. Events separated by more than one junction have independent relaxations and hence no interaction as in Eq. (93).

If two tunneling events that involve a common grain occur close together in time, the charge changes of the common grain can cancel and the combination is equivalent to a 
single pair-tunneling between further separated grains [see Fig. 8(c)]. We denote by $\xi_{n}$ the fugacity of a pair tunnel between grains separated by $s a$. Although in the original partition function (92), $\xi_{n}=0$ for $n \geq 2$, the long range pair tunnels will be produced during the RG flow.

The strength of the mutual and self interaction of pairtunnel events will determine whether they proliferate and induce superconductivity in the chain, or whether pairtunnels form bound pairs and annihilate each other, keeping the chain normal. For weak-Josephson-coupling, this can be analyzed via a perturbative RG analysis in powers of the $\left\{\xi_{s}\right\}$.

\section{B. RG flow of the pair-tunnel fugacities}

To study the RG flows, it is easiest to go back to the initial action in terms of the superconducting phase differences $\Delta_{j}$ between the junctions:

$$
\begin{aligned}
Z= & \int D[\phi(\tau)] \exp \left[-\frac{1}{2} \int \frac{d \omega}{2 \pi} \int \frac{d k}{2 \pi}\right. \\
& \times \frac{|\omega| a R_{Q}}{2 \pi\left[R+r\left(2-e^{i k a}-e^{-i k a}\right)\right]}\left|\Delta\left(k, \omega_{n}\right)\right|^{2} \\
& \left.+\sum_{i} \int d \tau \sum_{s} \xi_{s} \cos \left(\sum_{n=0}^{s-1} \Delta_{i+n}(\tau)\right)\right] .
\end{aligned}
$$

As explained in the previous section, this is equivalent to a Coulomb gas of pair-tunnel events because

$$
\xi_{s} \exp \left(\sum_{n=0}^{s-1} \Delta_{j}\right)=\xi_{s} \exp \left(\phi_{j+s}-\phi_{j}\right)
$$

increases the charge on grain $j+s$ by $2 \mathrm{e}$, and reduces the charge on grain $j$ by $2 e$ at rate $\xi_{s}$. We have explicitly included longer range tunneling as this will, in any case, be produced under renormalization from the basic nearest neighbor tunneling which has rate $\xi_{1}=E_{J}$. The dissipative interaction between a pair tunnel of range $s$ and a tunnel in the reverse direction a time $\tau$ later, is

$$
\frac{2 r+s R}{R_{Q}} \ln \left(\tau / \tau_{0}\right)
$$

proportional to the total resistance through which normal relaxation must occur to compensate for a Cooper-pair tunneling between grains separated by $s$.

The RG flows in powers of the fugacities of pair-tunnels are obtained similarly to those in the strong coupling regime in terms of QPS (see Sec. VIIB of Chap. 5 of Ref. 82): indeed they have a similar structure to the RG for the various QPS dipoles in the absence of individual QPS (Sec. IV H).

Including the combining of pair tunnel pairs-such as one from $j-s$ to $j$ together with one from $j$ to $j+m$ into a single one across $m+s$ junctions- the RG flows are

$$
\begin{aligned}
\frac{d \xi_{s}}{d l}= & \xi_{s}\left(1-\frac{2 r+s R}{R_{Q}}\right) \\
& +\tau_{0} \frac{1}{2} \sum_{m \neq s} \frac{2 r+m R+|s-m| R-s R}{R_{Q}} \xi_{m} \xi_{|s-m|} .
\end{aligned}
$$

Note that we have neglected the renormalization of two overlapping pair-tunnel events which do not share any grains. Such events renormalize into a complicated non-contiguous compound four-tunnel events, and are more strongly suppressed.

\section{Phase diagram and normal-FSC transition for weak coupling}

The trivial fixed point of the RG equations for the pairtunnel fugacities is the Gaussian line with no pair-tunnel events:

$$
\xi_{s}=0 \quad \forall s .
$$

This controls the low energy behavior of the normal phase of the chain. The normal fixed point is stable as long as all the $\left\{\xi_{s}\right\}$ are irrelevant: since $\xi_{1}$ is the least irrelevant, this obtains when: $\frac{2 r+R}{R_{Q}}>1$, so that with $R$ as the tuning parameter, the critical point in the limit of zero coupling is given by

$$
R_{M}^{0}=R_{Q}-2 r .
$$

When $R<R_{M}^{0}$, the normal fixed point becomes unstable: Cooper pair tunneling events proliferate and give rise to the superconducting phase. Since all the nonlinear terms in the RG flows of the $\left\{\eta_{s}\right\}$ are positive, these can only accelerate this flow so that the condition $R<R_{M}^{0}$ is certainly sufficient for superconductivity. Formally, the flow is then toward large $\xi$ and eventually to the FSC fixed manifold where the QPS fugacity, $\zeta$ is zero.

Because the nonlinear contributions to the flows are positive, superconductivity can still occur when $R+2 r>R_{Q}$ and the $\left\{\xi_{s}\right\}$ are irrelevant about the Gaussian fixed point. If

$$
\delta \equiv \frac{R+2 r}{R_{Q}}-1
$$

is small and positive, $\xi_{1}$ will be weakly irrelevant while the other $\left\{\xi_{s}\right\}$ are still strongly irrelevant. Nevertheless (as in Sec. IV H) the feedback of these to $\xi_{1}$ important. For small $\xi_{1}$ this is dominated by the creation of $\xi_{2}$ at order $\xi_{1}^{2}$, and the feedback of this into $d \xi_{1} / d \ell$ via the $\xi_{1} \xi_{2}$ term. There is thus a critical fixed point with

$$
\xi_{1}^{*} \sim \sqrt{\delta}
$$

and $\xi_{2} \sim \delta \ll \xi_{1}$. It can be seen that the longer range $\left\{\xi_{s}\right\}$ attain fixed point values $\xi_{s}^{*} \sim \delta^{s / 2}$. [Indeed, with the simple structure of the flows to quadratic order, an exact fixed point can be found: $\xi^{s} \propto(B)^{s}$ with $B$ an $r$ and $R$ dependent factor that is of order $\sqrt{\delta}$ for small $\delta$. But this is not a controlled expansion for the fixed point except when $\delta$ is in any case small, or perhaps in some special part of parameter space.] Equation (102) corresponds to a critical fixed line that controls the critical surface of the mixed character normal-FSC 

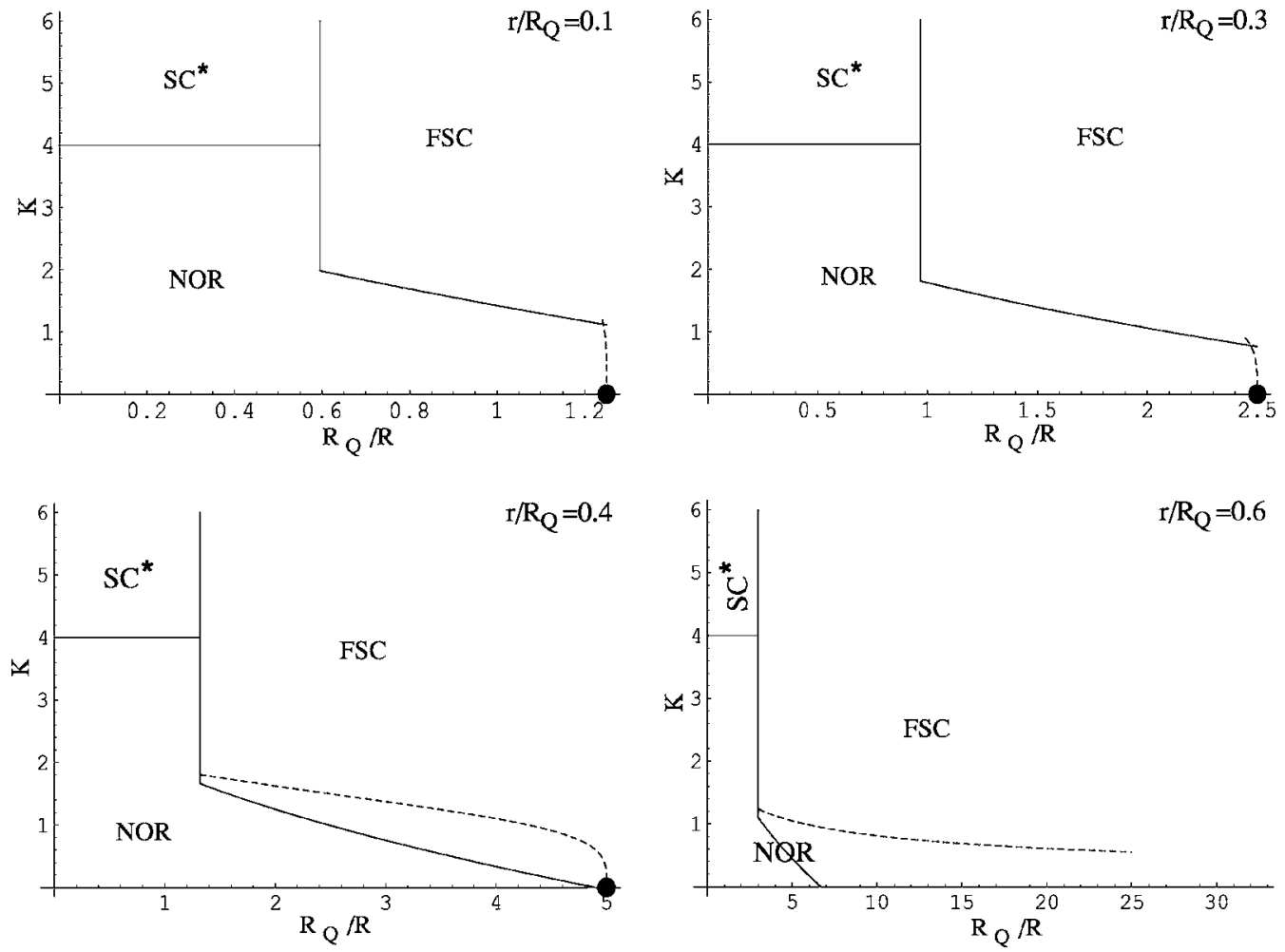

FIG. 9. Phase diagram with the weak Josephson coupling results. The solid lines represent the transition lines obtained with the strong coupling phase-slip approach (Sec. IV). The dotted line is obtained from extending Eq. (102) by solving the flow equations (98): $K$ $\sim \frac{1}{\sqrt{r}} \sqrt{\delta}$. The two approaches seem to cross into each other continuously. The meeting point of the weak and strong-coupling boundaries is likely to be a bicritical point where the transition changes its nature, but our methods are limited in that regime.

transition. For $\delta$ small, the critical $E_{J}^{c} \sim \xi_{1}^{c} \approx \xi_{1}^{*}(R, r)$ : when $E_{J}<E_{J}^{c}$ the chain is in the NOR phase, while when $E_{J}>E_{J}^{c}$ the chain is superconducting. Note that equivalently, one could pass from normal to superconducting phase at fixed Josephson coupling by decreasing $R$ through its critical value $R_{M}$, which, in the weak coupling limit is

$$
R_{M} \approx R_{Q}-2 r+\mathcal{O}\left(E_{J}^{2}\right) .
$$

Thus the Josephson coupling decreases the amount of dissipation that is needed to cause superconductivity. This is the same underlying physics as that makes the control parameter in the strong coupling limit $\frac{1}{2} K+\alpha$ which is the sum of a Josephson part and a dissipative part since $\alpha \propto 1 / \sqrt{R^{2}+2 r R}$. However, not surprisingly given the basic electrical properties of the chain discussed in Sec. II B, the combination of parameters that enter in the strong and weak coupling limits are quite different. In terms of the superconducting stiffness parameter $K=2 \pi \sqrt{E_{J} / E_{C}}$, the critical value of $K$ for the mixed transition is

$$
K_{M} \sim\left(R+2 r-R_{Q}\right)^{1 / 4}
$$

when this is small.

The critical behavior near the FSC-NOR transition will be characterized by the exponent $\mu$ with the crossover energy scale going to zero as $E_{\times} \sim\left|E_{J}-E_{J}^{c}\right|^{\mu}$. The critical exponent, $\mu$ is a continuously varying function of the resistances. For small $\delta$, the above analysis yields

$$
\mu \approx \frac{1}{2 \delta}
$$

The rapid fall-off with distance $s$ of the fixed point values $\xi_{s}^{*}$ of the pair tunneling amplitudes suggests that the fixed point does not have a long length scale associated with it, at least coming from the normal side. Whether there is a more subtle diverging length scale-e.g., that characterizes the decay with distance of the superconducting correlations, we leave for future investigation.

The important features of the weak-coupling phase diagram are shown in Fig. 9. When $2 r+R>R_{Q}$, the normal phase can exist; otherwise the chain will be fully superconducting. However, even for this high resistance regime, strong enough Josephson coupling can cause superconductivity. Along the phase boundary-actually a critical hypersurface in the parameters of our model-the critical behavior will vary continuously.

This analysis of the weak coupling regime thus almost completes the phase diagrams of Fig. 9. The one exception is the nature of the bicritical point at which the weak coupling section of the mixed-transition phase boundary meets the small-intermediate coupling KT section. As mentioned in the introduction, this does not appear to be amenable to perturbative analysis. A related question is whether, for intermediate coupling in terms of the QPS fugacities and pair tunneling amplitudes as well as $K$, the weak coupling segment of the mixed transition boundary, can meet the large- 
intermediate coupling dipole driven segment without an intervening KT section and, if so, do they come together smoothly or with another type of bicritical point? Again, this is not analyzable by our methods.

\section{NANOWIRES}

The discussion so far has concentrated on the phase diagram of a discrete chain of shunted Josephson junctions. One of the motivations for this work, however, came from the observation of superconducting-normal transitions in nanowires reported in Refs. 23, 24, and 89-91 (for earlier experiments see also Refs. 67-70). In this section we will show how one can describe the superconductor-to-normal transition in superconducting nanowires by carefully taking the continuum limit $a \rightarrow 0$ of the discrete model discussed before. Thermally activated phase slips in superconducting wires have been considered by Langer and Ambegaokar ${ }^{92}$ and by McCumber and Halperin. ${ }^{93}$ Quantum phase slips in superconducting wires have been discussed previously in Refs. 65-67 and 94-97.

The fundamental difference between a continuous wire and a discrete $\mathrm{JJ}$ chain is the existence of the lattice constant in the chain. The interplay between the lattice constant, $a$, and the charge relaxation length $\lambda_{Q}$ played an important role in JJ chains. In a wire, the characteristic dissipative length becomes

$$
\lambda_{Q}=a \sqrt{r / R}=\sqrt{\frac{a r}{R / a}}=1 / \sqrt{\gamma \rho},
$$

where $\gamma=\frac{1}{r a}$ is the phenomenological parameter describing the conversion conductance-per-length from the normal to superconducting fluids and $\rho=R / a$ is the resistance-perlength of the wire. Nanowires are often modeled as discrete chains of Josephson junctions with a lattice constant of the order of the superconducting coherence length $a=\xi{ }^{65}$ The motivation for this is that a typical size of a QPS should be of the order of $\xi$. However, from our discussion of JJ chains, it is clear that another important length is the smallest possible distance between QPSs. In the case of JJ chains it is $a$, but for continuous wires it may be arbitrarily small. So the correct description of the wire can be obtained from JJ models only by carrying out the $a \rightarrow 0$ limit with the coherence length $\xi$ fixed (see Fig. 10).

\section{A. Dipoles in nanowires}

Physically, the continuity of the wire and the limit $a \rightarrow 0$ allows the formation of dipoles of phase slips that are arbitrarily close to each other-up to charge discreteness effects which are discussed below. The closer together the phase slips are, the better their screening from the dissipative interaction, which is the only low energy effect that suppresses the proliferation of dipoles in a discrete JJ chain. This decreased dissipative interaction between them means that small dipoles in a wire will always proliferate. At zero temperature, a wire will thus always be either in the $\mathrm{SC}^{\star}$ phase or in the normal phase.

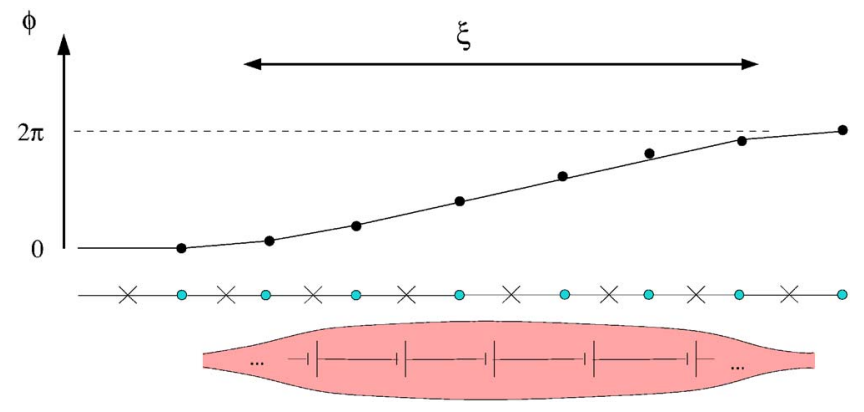

FIG. 10. (Color online) Schematic of a QPS in a continuous wire. The size of the core of a QPS-in which the superconductivity is suppressed-is of order the superconducting coherence length $\xi$. The wire can be considered as the limit of a chain of grains whose separation $a$ is much smaller than $\xi$.

This point can be made clearer by specifying how the continuum limit of a wire is taken. One method of taking this limit is spreading a phase slip over about $\xi / a \gg 1$ junctions, since a phase slip in a thin (diameter less than $\xi$ ) wire suppresses the superconducting order parameter over a length $\xi$. This can be taken into account in the sine-Gordon action by changing the cosine term in Eq. (48):

$$
\begin{aligned}
& \cos \left[\psi_{i}(\tau)+\theta_{i}(\tau)\right] \\
& \quad \rightarrow \cos \left[a \sum_{n} h(a n)\left[\psi_{i+n}(\tau)+\theta_{i+n}(\tau)\right]\right],
\end{aligned}
$$

where $h(x)$ is a weighting function concentrated in a region of length of order $\xi$ with the constraint

$$
\int d x h(x) \approx a \sum_{n} h(a n)=1
$$

This constraint on $h$ ensures that the total phase involved corresponds to one phase slip: it is forced by the global symmetry $\theta \rightarrow \theta+2 \pi$. The UV cutoff will no longer be set by the shortest lengths, but be of order $\Omega=c / \xi$ or the GinzburgLandau time, which is related to the gap (in a way that depends on whether the superconductivity is in the dirty or clean limit). Although the operator in Eq. (107) breaks a phase slip into parts that are fractions of $2 \pi$, it does not lead to independent fractional phase slips; because of the $2 \pi$ periodicity of the cosine, the fractional phase slips always appear together as a part of a complete phase slip. The structure of a smeared phase slip is shown in Fig. 10. The smearing means that the induced electrochemical potential drop caused by the phase slip is split into fractional potential drops that occur over roughly $\xi / a$ junctions adding up to the quantized drop of a single phase slip. The continuum limit $a \rightarrow 0$ can now be taken.

Because of the dissipation, an individual QPS in the wire will have action that diverges logarithmically at low temperatures, as in the chain. The effective dissipation parameter is given by 


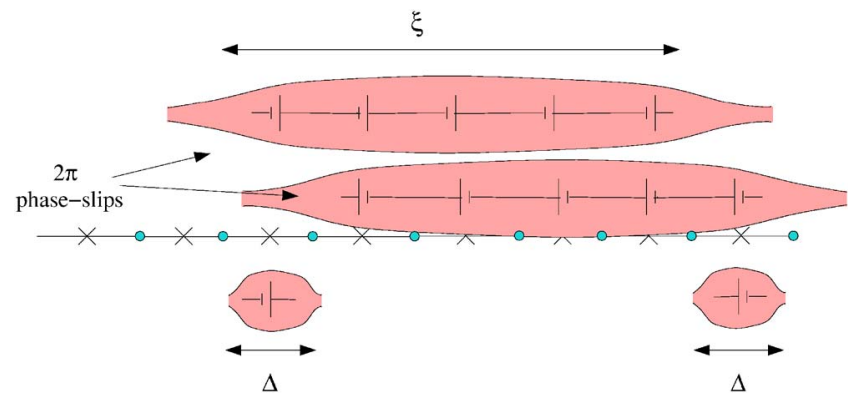

FIG. 11. (Color online) Dipoles formed of a phase-slip and antiphase-slip that are a distance $\Delta \ll \xi$ apart. Their net effect is two partial phase slips of size $\Delta$, a distance $\xi$ apart.

$$
\alpha=\int d x h(x) \int d y h(y) \frac{1}{2} \sqrt{\frac{\gamma}{\rho}} e^{-|x-y| / \lambda_{Q}} .
$$

There are now two limits of interest. If $\lambda_{Q} \ll \xi$ (but still $\lambda_{Q} \gg a$ so that the discreteness does not matter) the parameter is

$$
\alpha \sim \frac{R_{Q}}{\rho \xi}
$$

with the coefficient depending on the form of $h(x)$ as should be expected: there is no unique way of defining the core size of a phase slip. This result involves the normal resistance over a distance $\xi$ : thus in the wire $\rho \xi$ (rather than $\rho a$ ) plays the role of $R$ in the JJ chain. However, in general, $\rho \xi$ is not the relevant resistance, in contrast to what would have been guessed from this analogy.

If $\lambda_{Q} \gg \xi, \xi$ does not play a role in the dissipation and the behavior is similar to the $\mathrm{JJ}$ chain for $\lambda_{Q} \gg a$ so that

$$
\alpha \approx \frac{1}{2} R_{Q} \sqrt{\frac{\gamma}{\rho}}
$$

analogous to $1 / 2 \sqrt{r R}$ for the chain. In this limit, $\alpha$ will be of order unity when the normal resistance of a length $\xi$ of the wire is still small.

By analogy with the JJ chain, one would expect that the condition for breakdown of superconductivity would be controlled by the coefficient of the total interaction between QPS at the same position but different times $K+2 \alpha$. From the arguments of Sec. IV F, one would expect the critical value of this coefficient to be three. Indeed, there should be a change in the behavior near when this condition is met, but it will not be a true transition because of the role of dipoles.

A pair of opposite sign phase slips separated by a distance $\Delta$-a dipole with moment $\Delta$-will have infinite action at zero temperature because of the dissipation. However, the dissipative interactions between the QPS that extend out to $\Delta$ of order the greater of $\lambda_{Q}$ and $\xi$ will reduce the coefficient, $\beta(\Delta)$ of the logarithmic action of the dipole (also see Fig. 11).

As in the chain, a pair of equal and opposite dipoles with the same location but separated in time will have action

$$
2 \beta(\Delta) \ln \left(\tau / \tau_{0}\right)
$$

with the dissipative parameter a function of the resistances and the dipole moment $\Delta$ relative to $\xi$ and $\lambda_{Q}$. In the limit that $\Delta \ll \min \left(\xi, \lambda_{Q}\right)$, for smooth weighting function $h$, the action can be expanded in powers of $\Delta$ yielding

$$
\beta \sim \frac{R_{Q}}{\rho \xi} \frac{\Delta^{2}}{[\max (\lambda, \xi)]^{2}} .
$$

For $\xi \ll \lambda_{Q}$, there will a change in behavior from quadratic to linear dependence on $\Delta$ for $\Delta \sim \xi$ : the larger moment behavior then being like the JJ chain with $\lambda_{Q} \ll a$ so that $\beta$ saturates to $\approx 2 \alpha$ at $\Delta \sim \lambda_{Q}$. For $\xi \gg \lambda_{Q}, \beta$ will crossover from quadratic to saturating at $\Delta \sim \xi$.

Because $\beta$ decreases to zero as $\Delta \rightarrow 0$, independent of the dissipative parameters or of $\xi$, for sufficiently small $\Delta, \beta$ will be less than unity and thus dipoles will proliferate-as long as very small dipoles can be modeled as we have done here. The characteristic moment $\Delta$ below which dipole proliferation occurs is

$$
\Delta_{\times} \sim \max \left(\lambda_{Q}, \xi\right) \sqrt{\rho \xi / R_{Q}}
$$

provided this is less than $\xi$, otherwise a different dependence obtains. Nevertheless, we see that independent of the relative magnitudes of $\xi$ and $\lambda_{Q}$, dipoles of moment $\xi$ will not proliferate as long as $1 / \sqrt{\rho / \gamma}<C_{h} R_{Q}$ with $C_{h}$ an order-unity coefficient that depends on details of the structure on scale $\xi$.

If dipoles of size of order $\xi$ can proliferate, the behavior will be similar to the JJ chain when $s=1$ dipoles, the smallest size, proliferate. The dipole proliferation will, at low energies, lead to screening of the dissipative interactions between individual QPS and thus to either the $\mathrm{SC}^{\star}$ phase or the normal phase depending on whether the renormalized $K$ is greater than or less than 4 .

However, if only dipoles of size $\Delta \ll \xi$ can proliferate on their own, the behavior will be quantitatively different. Although it is not clear what pairs of QPS whose separation is much less than $\xi$ represent, they should correspond to large localized amplitude fluctuations of the superconductivity that act to decouple the superconducting from the normal degrees of freedom. The effects of these will be to renormalize the dissipative interactions between larger moment dipoles and individual QPS by decreasing the rate of the processes, parametrized by $\gamma$, that relax imbalances between the superconducting and normal voltages. Again, if the dipole moments can be arbitrarily small, this will eventually lead to either the $\mathrm{SC}^{\star}$ or the normal phase. But if the bare rate of the smalldipole-like processes $\eta(\Delta)$ is small, there should be interesting crossovers as temperature is lowered associated with the growth at low energies of these dipole fugacities before they fully screen the dissipative interactions.

In principle, even in a chain of grains linked by Josephson junctions QPSs need not take place only across the junctions but could happen inside a grain - albeit with much higher action and hence exponentially lower fugacity. However, in the limit of extremely low temperatures, such processes need to be considered and the chain will be more similar to a nanowire. In particular, the effects of QPS dipoles with very small size within one grain will, by the arguments above, be 
relevant. Eventually, these will screen the superconducting fluctuations from the dissipative interactions and drive the system into the $\mathrm{SC}^{\star}$ or normal phases: again, if arbitrarily small dipoles can occur, the FSC phase will not exist in the limit of asymptotically low temperatures.

\section{B. Minimum size QPS dipoles?}

In all the above, we have assumed that arbitrarily small QPS dipoles can exist: in the continuum model we have used, even with short distance cutoffs, there seems to be no reason these cannot occur. However, as the QPS are quantum objects, we must be careful of the possibility of complexrather than purely real-actions.

In a QPS dipole, the superconducting phase, $\phi(x)$, inside the dipole winds by $2 \pi$ as $\tau$ goes from $-\infty$ to $+\infty$ (see Sec. III B). As long as $\phi(x, \tau)$ is periodic in $\tau \in(0, \beta)$, a QPS dipole of the opposite sign is needed to satisfy this condition. In reality, however, the true condition is that $\phi$ is periodic in 0 to $\beta$ modulo $2 \pi$. In the limit of zero temperature, this will have no effect in the action we have studied. But in general, there is an additional term in the action, analogous to a Berry phase, of the form

$$
S_{w}=i \int d x \int_{0}^{\beta} d \tau \tilde{n}_{S} \frac{\partial \phi}{\partial \tau},
$$

with $\widetilde{n}_{S}$ having units of number density. In a Galilean invariant system, at zero temperature $\widetilde{n}_{S}$ would be the density of Cooper pairs, equal to half the electron number density. More generally, it can have any value, positive, negative, or zero: $\widetilde{n}_{S}$ is some effective density for the superfluid that has no known simple interpretation. It will depend on particlehole asymmetry and other factors that are not understood. ${ }^{98,99}$ For vortices in a $2 \mathrm{D}$ film, $\widetilde{n}_{S}$ corresponds to a dual magnetic field, and it gives rise to a vortex hall effect. ${ }^{100}$ (Note that in Ginzburg-Landau theory, only the combination $n_{S} / m^{*}$ is physical: $n_{S}$, in contrast to the phase stiffness, $Y$-often called $\rho_{S}$-has no physical meaning. Quantum mechanically, this is no longer true.)

The action of a dipole of moment $\Delta$ has an imaginary part of its action from the Berry phase term

$$
S_{w}^{\text {dipole }}=2 \pi i \tilde{n}_{S} \Delta .
$$

This suggests that dipoles for which $\widetilde{n}_{S} \Delta$ is not an integer will undergo destructive interference implying that $\Delta$ should be quantized in units of $1 / \widetilde{n}_{S}$. The QPS dipoles can be considered as a vortex which first crosses the wire in one direction, and then returns and cuts the wire in the opposite direction at a distance $\Delta$ away from the first crossing, before returning to its origin. Interpreting $\tilde{n}_{S}$ as Cooper pair density, the quantization of $\Delta$ means that the vortex had to "go around" integer numbers of Cooper pairs. ${ }^{101}$

If there is indeed a minimum size QPS dipole with

$$
\Delta_{\min }=\frac{1}{\widetilde{n}_{S}},
$$

and the low frequency coupling to the "normal" degrees of freedom is still effectively dissipative for such small dipoles, then it is possible that the dissipation could be sufficiently strong to suppress these smallest dipoles. [Although, the effective coupling $\beta\left(\Delta_{\min }\right)$ would be determined by the shorter length scale physics which will not be well parametrized by $\gamma$ and $\rho$.] If the smallest dipoles are indeed suppressed, then an FSC phase-and a transition from it to an $\mathrm{SC}^{\star}$ or a normal phase-would be possible in nanowires.

It is worth noting that effects of a small local disturbance can be long range in time and give rise to actions that diverge logarithmically as temperature is lowered. An example is an impurity in a metal that is moved by a small amount: the divergent action is associated with the "orthogonality catastrophe" caused by the changes in the electron wave functions induced by the altered potential. ${ }^{102}$ Better known, but closely related, is the $\mathrm{x}$-ray edge singularity. ${ }^{103}$ Thus it is not unreasonable to think that a local change in the superconducting degrees of freedom could result in a logarithmically infinite action associated with the response of the quasiparticles and other low energy degrees of freedom to the change. Whether or not the FSC phase can occur, even in principle, we leave as an intriguing open question.

\section{RESISTIVITY}

In the previous sections we concentrated on the zero temperature phase diagram of the RSJJ chain shown in Fig. 1(c). We now extend this analysis to discuss the temperature dependence of the resistivity of the system using the RG flow equations obtained in Sec. III D. One of the most surprising results is that in the normal phase close to the superconductor-to-normal transition the system exhibits a nonmonotonic $R(T)$ dependence. In this regime one finds that the resistivity first decreases with decreasing temperature, then saturates and stays nearly constant at a value $R(T) \ll R$, i.e., much smaller than the normal resistance of the chain, down to very low temperatures, and only then finally starts to increase. During this long crossover, the Josephson junctions themselves form a resistive channel, parallel to the shunt resistors. Since experiments have a lower limit of temperature, such behavior may appear to support the existence of the "metallic phase"14 separating the superconducting and insulating phases.

We start by showing how one can obtain the resistance vs temperature curves from the RG flow for the QPS fugacity. We then proceed to discuss the finite temperature resistivity in the $\mathrm{FSC}$ and $\mathrm{SC}^{\star}$ regions, and explain the origin of the quasimetallic behavior in some of the NOR region.

\section{A. Resistance of a single junction}

The RG analysis presented in this paper allows one to calculate the scaling behavior-including crossovers-of the temperature dependence of the resistance of a JJ chain. The simplest way to do this is to carry out the RG flow until the UV cutoff becomes of order the temperature. At lower energy scales the renormalized phase slips are only weakly interacting and therefore can be considered independent.

To obtain physical quantities, we must distinguish between the "bare" parameters - those that appear in the action 
with the initial high energy cutoff - and the renormalized parameters. In situations in which there might be confusion, we denote the renormalized parameters with subscripts $\ell$ corresponding to energy scale $\Omega=\Omega_{0} e^{-\ell}$.

We first consider a single resistively shunted Josephson junction and estimate the (superconducting) voltage fluctuations across it at a low temperature $T$. At low frequencies, the resistance of the superconducting part controls the low frequency fluctuations via

$$
\left\langle V(\omega) V\left(\omega^{\prime}\right)\right\rangle=R T 2 \pi \delta\left(\omega+\omega^{\prime}\right) .
$$

The voltage fluctuations caused by QPS are tricky to estimate because of the interactions between them. However, if we renormalize until an energy scale at which the QPS are weakly interacting, $T(\ell) \sim \Omega$, then the fluctuations can be estimated simply.

The phase-slip fugacity $\zeta_{\ell}$ and the temperature of a single RSJJ obey the flow equations ${ }^{88}$

$$
\begin{gathered}
\frac{d \zeta_{\ell}}{d l}=\zeta_{\ell}\left(1-\frac{R_{Q}}{R}\right), \\
\frac{d T_{\ell}}{d l}=T_{\ell}
\end{gathered}
$$

with the high energy cutoff $\Omega_{0}$ initially of order the plasma frequency of the circuit. When the RG flow reaches the stage in which $T_{l} \sim \Omega$, only one pair of phase slips (they must come in pairs because of the periodic boundary conditions in $\tau)$ is likely in the rescaled imaginary time duration $1 / T_{\ell}$ and these are, in any case, weakly interacting. At this scale, the renormalized fugacity is

$$
\zeta_{T}=\zeta\left(\ell=\ln \left[\Omega_{0} / T\right]\right) .
$$

The phase slip pairs will then occur with probability of order $\zeta_{T}^{2} / \Omega^{2}$ in time of order $\hbar / T_{\ell} \sim \Omega$. A phase slip contributes to $V(\omega=0)$ - a quantity that is scale independent-an amount $h / 2 e$. The rescaling of $T$ and of $\delta\left(\omega+\omega^{\prime}\right)$ in Eq. (118) cancel, and the renormalization of $\zeta$ to $\zeta_{T}$ then yields

$$
R_{J J} \sim \frac{h}{(2 e)^{2}}\left(\zeta_{T} / \Omega\right)^{2} \sim R_{Q}\left(\zeta \Omega_{0}\right)^{2}\left(\frac{\Omega_{0}}{T}\right)^{2-2 \alpha} .
$$

The resistance increases with decreasing temperature in the normal phase which obtains when $R_{Q} / R<1$, and goes to zero at zero temperature in the superconducting phase which obtains when $R_{Q} / R>1$. This result, Eq. (121), is valid only for $T<\Omega_{0}$. For higher temperatures, $T>\Omega_{0}$ activated scaling of the form $R_{J J} \sim R_{Q} \exp \left(-C_{J} E_{J} / T\right)$ with $C_{J}=\mathcal{O}(1)$ obtains. How these match together can be understood in terms of the fugacity $\zeta$. This is of order $\Omega_{0} \exp \left(s_{0} / \hbar\right)$ with $s_{0}$ the action of a phase slip $s_{0} \sim E_{J} \tau_{0} \sim E_{J} / \Omega_{0}$ since the time scale of a QPS is $\tau_{0}$. If the imaginary time $\hbar / T$ is smaller than $\tau_{0}$, the action of the phase slip will be reduced by a factor of order $\hbar / T \tau_{0}$. This gives rise to the activated resistance in terms of $E_{J} / T$.

The measured resistance of the shunted junction is

$$
R_{m}^{-1} \approx\left(R_{J J}\right)^{-1}+R^{-1} .
$$

If in Eq. (121), $\zeta \sim \Omega_{0} \frac{R}{2 \pi R_{Q}}$ or larger, the Josephson junction becomes essentially insulating, and most of the current flows through the shunt resistor $R$.

Note that at any nonzero current, some Cooper-pair tunneling will occur and nonlinear resistance $V / I$, will no longer be given by Eq. (122). However, these corrections vanish in the limit of zero temperature and zero current.

\section{B. Resistivity in the FSC region}

We now turn to the JJ chain. In the FSC phase, each junction behaves similarly to a single junction in its superconducting phase and thus contributes roughly independently to the total resistance. Phase slip dipoles, with fugacities $\left\{\eta_{s}\right\}$, are the explicit manifestation of interaction between phase slips on different junctions. Thus when all the $\left\{\eta_{s}\right\}$ are irrelevant about the FSC fixed line, the phase slips in each junction will be almost independent-although their dissipation involves overlapping resistors. Thus each Josephson junction will act similar to the a single junction with resistance given by Eq. (122), with the appropriate $\zeta_{T}$-the renormalized fugacity at scale $T \sim \Omega$ - of individual QPS in the chain

$$
R_{\mathrm{JJ}} \sim \frac{h}{(2 e)^{2}} \tau_{0}^{2} \zeta_{T}^{2} .
$$

At intermediate temperatures (what we mean by intermediate is discussed in Sec. IV F), Eq. (123) predicts a measured resistance per unit length

$$
\rho_{m} \approx R_{J J} / a \sim T^{\Gamma}
$$

with the (positive) exponent $\Gamma$ depending on the normal resistances and the superconducting stiffness $K$.

In the FSC phase, the exponent $\Gamma$ is given by minus twice the eigenvalue $\zeta$ [from Eqs. (80)]

$$
\Gamma=K+2 \alpha-2 .
$$

Since the boundary between the FSC and normal phase when the $\left\{\eta_{s}\right\}$ are irrelevant-the mixed transition-is given by $K_{M}=3-2 \alpha$, we see that on the critical line

$$
\rho_{m}^{M} \sim \frac{R_{Q} T}{a \hbar \Omega_{0}}
$$

in contrast to the single junction critical point at which the measure resistance will be roughly temperature independent. The origin of the factor of $T$ can be seen from the transformation that related the flow of $\zeta_{\ell}$ to the flow of the parameters $z \propto \zeta_{\ell} \exp (-\ell / 2)$ and $x=\frac{K}{2}+\alpha-\frac{3}{2}$ which obey the Kosterlitz Thouless flows Eqs. (84). When $\zeta$ is small, $x=0$-at which $z$ is weakly scale dependent-marks the FSC-NOR transition. The scale dependence of the relationship between $z$ and $\zeta_{\ell}$ transforms Eq. (123) to $R_{\mathrm{JJ}} \sim R_{Q} \tau_{0}^{2} \zeta_{T}^{2} \sim R_{Q} \frac{T}{\Omega} z_{T}^{2}$ giving rise to the factor of $T$ in terms the "natural" variable $z$. As we shall see, this really is natural for the KT-like global transition from the $\mathrm{SC}^{\star}$ phase to the normal phase.

The behavior of $R_{\mathrm{JJ}}(T)$ near the FSC-NOR transition is shown in Fig. 12(b). The results in Eqs. (80)-(82) lead to a 

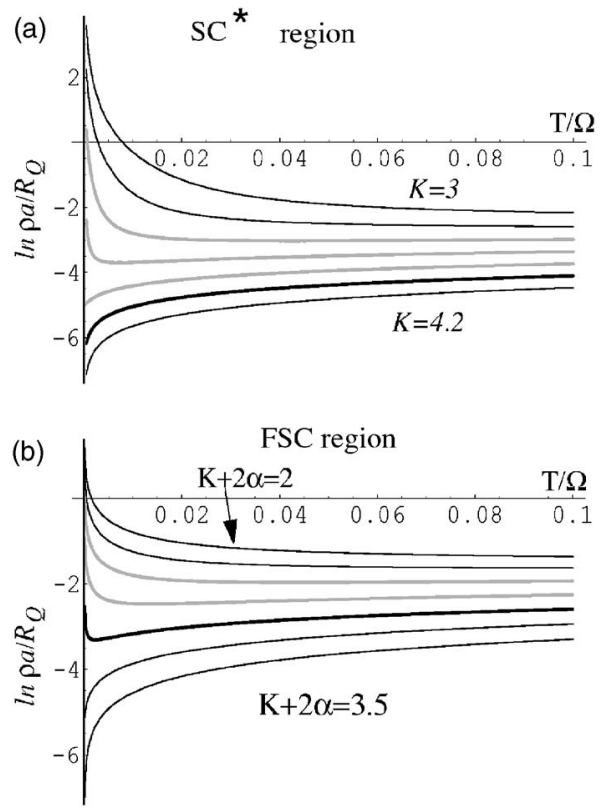

FIG. 12. Semilog plot of the resistance vs. temperature for various values of $K$. (a) Resistivity in the $\mathrm{SC}^{\star}$ phase. $K$ $=3,3 \cdot 2,3 \cdot 4,3 \cdot 6,3 \cdot 8,4,4.2$. (b) Resistivity in the FSC phase. $K$ $+2 \alpha=2,2.25,2.5,2.75,3,3.25,3.5$. In both plots the resistivity at the bare critical value of the stiffness appears as a thick black line $(K=4$, and $K+2 \alpha=3)$. The nonzero phase-slip fugacity shifts these lines slightly from criticality. Resistivities in the nonmonotonic quasimetallic region are shown as thick gray lines. Temperature is normalized by the plasma frequency of an individual junction. The contribution of the parallel channel of normal electrons to the total conductivity has been subtracted. We use the approximation $\zeta$ $\sim e^{-\sqrt{8} K / \pi}$ as initial condition, which corresponds to the bare action of the QPS $S_{0}=8 \sqrt{E_{J} / 2 E_{C}}$ (Refs. 36 and 104).

regime of parameters in which a crossover between quasisuperconducting and normal behavior takes place: in particular, the resistance exhibits a minimum at a low temperature. This behavior arises on the normal side of the transition, but still close to it: when

$$
1<\frac{K}{2}+\alpha<\frac{3}{2}
$$

so that $\zeta_{\ell}$ initially decreases with scale even though $z$ is increasing. If the bare fugacity is small, this gives rise to an intermediate temperature regime with a power law decreasing resistance with exponent $0<\Gamma<1$. However, at low enough energies, the rapid increase of $z$ will give rise to proliferation of dipoles and individual QPS and the $\zeta_{\ell}$ will start to grow. The junction then crosses over to insulating behavior as the temperature is lowered further. Near the transition to the FSC phase, the crossover temperature $T_{\min }$ at which the resistive minimum occurs becomes very low:

$$
T_{\min } \sim \Omega_{0} \exp \left(-b / \sqrt{K_{M}-K}\right)
$$

with $b$ a coefficient proportional to the bare QPS fugacity $\zeta$ when this is small. Below the crossover temperature, the measured resistivity $\rho_{m}$ of the chain will increase as the tem- perature is lowered, until it reaches the resistance of the shunt resistors $R / a$.

\section{Resistivity in the $\mathrm{SC}^{\star}$ region}

The low temperature behavior in the $\mathrm{SC}^{\star}$ phase can be understood similarly. When the renormalized dipole fugacities are large, dissipation will be screened and we need only consider individual QPS and the interactions between them mediated by the superconducting degrees of freedom.

It is now simplest to use the isotropic RG and rescale space and time. When the RG flow reaches the stage where $T(\ell)=\Omega$, the spatial size of a QPS is $\Omega_{0} / T$ times its original size. Therefore, when resistances per unit length are found from renormalized quantities, a factor of the length rescaling is needed to obtain the physical resistivities. The resistivity of the Josephson junctions, will thus be

$$
\rho_{\mathrm{JJ}} \sim \frac{T}{\Omega_{0}} \frac{R_{Q}}{a}\left(\zeta_{T} / \Omega\right)^{2} \sim \frac{R_{Q}}{a}(\zeta / \Omega)^{2}\left(\frac{\Omega_{0}}{T}\right)^{\Gamma},
$$

with

$$
\Gamma=1+(K-4),
$$

where the $K-4$ is minus twice the eigenvalue of $\zeta$ in the isotropic RG. We have assumed that the basic length scale that enters is the grain spacing $a$ and used the bare QPS fugacity $\zeta$ : more generally these will depend on the higher energy processes as discussed below.

The resistivity as a function of temperature is plotted for several values of $K$ in Fig. 12(a). Since for $K<K_{G}=4$ the chain will be normal at low temperatures and the resistivity increase to $R / a$, for the intermediate range

$$
3<K<4
$$

the resistivity will decrease with decreasing $T$ and have a minimum at a crossover temperature $T_{\min }$ given by the energy scale at which the QPS proliferate enough to screen the longer-range interactions between them driving the junctions normal. This range is analogous to the intermediate regime of Eq. (127) near the FSC phase: In both cases the resistivity seems to show signs of superconductivity before exhibiting insulating behavior. For $K \approx 3$ the flat resistivity curves over a substantial temperature range could be confused for a quasimetallic phase.

\section{Roles of dipoles}

In the JJ chain, if the QPS fugacities are small and the dissipative interactions substantial, the QPS fugacity can be irrelevant, but the dipoles may be relevant about the FSC fixed line. In this case, the $\mathrm{SC}^{\star}$ phase behavior can obtain only at low enough energies below which the QPS dipoles proliferate: this makes the temperature dependence of the resistivity much richer than that discussed so far. If the $\mathrm{SC}^{\star}$ phase is not stable, the resistivity near the dipole driven FSCNOR transition, will involve a crossover from dipole to individual QPS dominated resistivity.

The temperature behavior of the resistivity in both these cases splits into two regimes. Since the $\left\{\eta_{s}\right\}$ are zero initially, 
and get generated at order $\zeta^{2}$, at temperatures in the range $T_{D}<T<\Omega_{0}$ we expect the JJ chain to behave as though it were in the FSC region. The crossover temperature $T_{D}$, away from the FSC regime is the energy scale at which $\eta_{1}$ becomes of order $\Omega$ : at lower energies, the dissipative interactions get screened [see discussion above Eq. (75)]. At energies higher than $T_{D}$, the scaling is similar to that of individual junctions, while at lower temperatures, it will become isotropic as in the $\mathrm{SC}^{\star}$ regime discussed above, and exhibit normal behavior at sufficiently low energies if $K$ is too small to stabilize the $\mathrm{SC}^{\star}$ phase.

We now estimate $T_{D}$, and discuss its consequences for the low temperature behavior. The flows of $\eta_{1}$ when it is relevant can be well approximated by an initial rapid regime in which $\eta_{1}$ becomes of order $\zeta^{2} / \Omega_{0}$, followed by a scaling regime in which it grows with exponent $1-\beta$ with $\beta$ $=2 \alpha(1-w)$ as in Sec. IV H. Thus it will become of order $\Omega$ at an energy scale

$$
T_{D} \sim \Omega_{0}\left(\frac{\Omega_{0}}{\zeta}\right)^{2 /(1-\beta)}
$$

for $\beta$ below its critical value of unity for either the FSC-SC ${ }^{\star}$ or the FSC-NOR dipole-driven transitions. This is valid as long as $\zeta_{\ell}^{2}$ grows less rapidly than $\eta_{1}$, i.e., if $2-2 \alpha-K<1$ $-\beta$. This is always the case if $K>1$ and sometimes also at smaller $K$ if $w$ is substantial. If $\zeta_{\ell}^{2}$ grows more rapidly than $\exp [(1-\beta) \ell]$, then $\eta_{1}$ follows $\zeta_{\ell}^{2}$ and individual QPS will drive the crossover to normal behavior.

For $T>T_{D}$, the individual-junction controlled behavior found above for the resistivity in the FSC phase (and for crossover away from FSC) will obtain

$$
R \sim T^{K+2 \alpha-2} .
$$

However, below $T_{D}$ the temperature dependence of the resistivity will be like that in the $\mathrm{SC}^{\star}$ phase (or the flow away from that) with $\rho_{m} \sim T^{K-3}$. As before, if $K<4$, at low enough temperatures the chain will crossover to normal behavior and the resistivity saturate at $R / a$, while if $K>4$ the resistivity will go to zero more rapidly than linearly.

We have seen in this section that the interplay between local and long-length scale physics gives rise to interesting behavior of the resistivities. Perhaps surprisingly, the temperature dependence near the critical point of the QPS driven mixed transition, for which the critical value of the parameter $\frac{1}{2} \kappa+\alpha$ is $\frac{3}{2}$, midway between the values of 1 and 2 expected for local and global transitions, nevertheless is similar to that of the global transition with $\rho_{m} \sim T$, with the $T$ arising from spatial rescaling.

Unfortunately, the form of the various crossovers of the resistivity as a function of temperature, which would yield firmer testable predictions, are not readily accessible to our analysis since these involve analyzing a sine-Gordon model in the intermediate coupling-strength regime. In addition, we have been rather cavalier about which resistivity we are considering: in general, even in simple scaling regimes, the resistivity at $\omega \sim T$ and the dc resistivity can be quite different. The latter certainly requires proper real-time analysis.

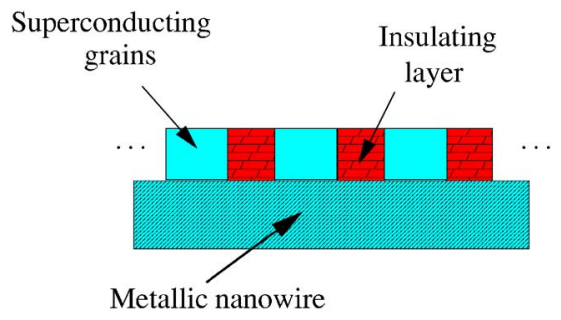

FIG. 13. (Color online) Possible realization of a two-fluid JJ chain: mesoscopic superconducting grains deposited on top of a metallic nanowire and separated by a thin insulating layer, to produce an SIS junction.

\section{DISCUSSION}

In this final section we discuss some implications of the results presented in this paper, and issues associated with these.

\section{A. Chains of mesoscopic Josephson junctions}

Several groups have recently reported experimental realizations of mesoscopic superconducting grains ${ }^{105}$ and arrays of low-capacitance Josephson junctions. ${ }^{19,35,36,48-52}$ The possibility of introducing ohmic dissipation using shunt resistors has been demonstrated for a single quantum Josephson junction by Pentillä et al., ${ }^{36}$ and Watanabe and Haviland, ${ }^{19}$ and for arrays of Josephson junctions by Takahide et al..$^{54}$ and Miyazaki et al. ${ }^{58}$ A potential realization of a chain of mesoscopic Josephson junctions with ohmic dissipation is shown in Fig. 13. It consists of equally spaced small superconducting grains on top of a metallic nanowire with thin insulating layers separating the two materials. This system is a realization of the model that we presented in Sec. III, with the possibility of controlling separately the parameters $E_{J}, E_{C}, R$, and $r$ by changing the width of the metallic wire, the size and separation of the superconducting grains, and the strength of the tunnel barriers between the grains and between the grains and normal wire. A schematic phase diagram of this system is presented in Figs. 7 and 9 (we did not consider odd-even effects in the grains, which must be irrelevant if $r<R_{Q}$, as discussed below). Both the phase diagram and the character of the transitions are affected by the superconducting-normal relaxation parametrized by $r .{ }^{106-108}$

If the metallic wire is highly resistive and/or the tunnel barrier between the $S$ (superconducting) and $N$ (normal) parts weak, the dissipation will not be important, the FSC phase will not occur, and the superconductor-to-normal transition will be determined primarily by the competition between Josephson coupling and Coulomb charging energy of the grains. This will be a quantum Kosterlitz-Thouless transition, isotropic in space and time, with the energy scale decreasing exponentially as the transition is approached.

If the wire is less resistive and/or the $\mathrm{SN}$ tunnel junction stronger, the FSC phase can occur. The FSC to normal phase boundary and the FSC to $\mathrm{SC}^{\star}$ phase boundary will be dominated by dissipative effects. These change, in particular, the nature of the superconductor-to-normal transition which will have a mixed character with both local and global physics 
involved. This mixed transition can either be KT-likealthough modified from the conventional KT transition-or have strongly anisotropic power law scaling with continuously variable exponents.

The renormalization group analysis presented in this paper allows us to obtain the scaling form of the chain's resistivity as a function of temperature in the vicinity of the various superconductor-to-normal transitions; the results are presented in Fig. 12. Near the $\mathrm{SC}^{\star}$ to normal phase boundary, our results agree with the results in Refs. 65 and 66, but the analysis of $R(T)$ in the vicinity of the FSC-to-normal phase boundary is new. We find that in both cases the system has extended crossover regions on the normal side of the transition, in which superconducting tendencies give rise to a weakly temperature dependent resistivity that decreases as a small power of $T$ over a wide temperature range before turning up at the lowest temperatures as it becomes asymptotically normal. Near the superconductor-to-normal phase boundary this upturn in $R(T)$ occurs at temperatures that are either power-law or exponentially small in the inverse of the deviation from the zero-temperature transition, depending on the regime.

Our RG approach to the problem allowed us to expose a myriad of crossover effects occurring near phase boundaries. Particularly interesting is the interplay between global and local mechanisms for the breakdown of superconductivity. The mixed FSC-normal transition, for example, can arise from the local resistive environment, or from a combination of the long-wavelength plasmon dissipation and the local resistive parts. The phase boundaries for which these two mechanisms obtain meet at a bicritical point, which has a rich crossover behavior yet to be analyzed in full. The $\mathrm{SC}^{\star}$-normal transition is shown to be global, i.e., determined by the long-wavelength excitations, and thus, for an infinite chain, the low energy critical behavior is almost independent of the resistive shunting or the two-fluid nature of the grains, except for their effects in modifying the effective capacitances and Josephson couplings.

Other aspects of the phases and phase transitions could also be investigated for a chain of grains. Of particular interest would be tunneling into pairs of grains, studying the behavior as a function of their separation and of temperature, and how these change near phase transitions.

\section{B. Superconducting nanowires}

Various types of superconducting nanowires can have dissipation down to zero temperature. The simplest is perhaps a wire with regions that remain normal, so that it has a positive density of states at zero energy. Another possibility is a sandwich of superconducting and metallic nanowires separated by a thin insulating barrier (see Fig. 14). However, even nanowires made of a conventional gapped superconductor may have low energy degrees of freedom that give rise to dissipation when they are close to a superconductor-tonormal transition. The conventional BCS theory of superconductivity, which leads to an energy gap, is intrinsically based on a mean-field analysis and thus unlikely to be reliable in the vicinity of a quantum phase transition when virtual quan-

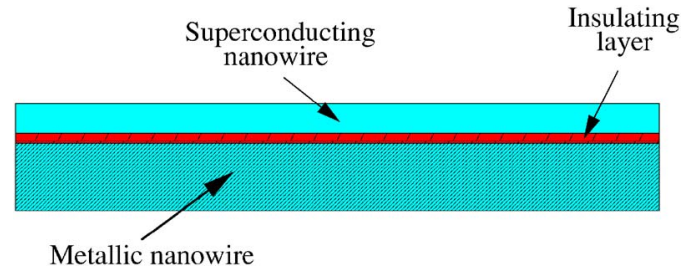

FIG. 14. (Color online) Schematic of a type of superconducting nanowire with dissipative degrees of freedom: a superconducting layer is deposited on top of a metallic nanowire with an insulating layer in between.

tum phase slips occur. A roughly analogous effect is seen in superfluid ${ }^{4} \mathrm{He}$ in a disordered porous medium (e.g., Vycor): an apparent normal fluid component has been observed down to the lowest temperatures. ${ }^{109}$ In general, one would expect dissipation of some sort—but not necessarily Ohmic — near super-

conductor-to-normal quantum transitions as long as some of the Cooper pairs break apart near the transition. This may not occur if the Cooper pairs form tightly bound bosons that still exist in the nonsuperconducting phase, but more generally should occur. Furthermore, if the normal phase is a Bose or Fermi glass, there will be a constant density of states of low energy excitations and concomitant dissipation arising from local two level systems. That said, the approach we have taken in this paper is phenomenological: we assume that dissipation is present in at least some superconducting nanowires and then study its consequences.

For superconducting nanowires, our analysis of the simplest model suggests that nanowires will always be either in the $\mathrm{SC}^{\star}$ or the normal phase with the FSC phase unstable to small QPS dipoles. The superconductor-normal transition would then generally be of global KT character. However, if the fugacity of phase slips is low, and the dissipative effects large, there will be interesting crossovers in the temperature and length-scale dependent properties in both the superconducting phase, and near the transition.

Nevertheless, as discussed in the Sec. VI, these conclusions may not be correct. In particular, charge discreteness effects can lead to interference between QPS dipoles which would set a minimum size for dipoles in the limit of zero temperature. If the dissipation is strong enough - a condition that will depend on short-length scale physics in addition to the mesoscopic resistive parameters - then it is possible that the FSC could be stabilized. These effects clearly need further exploration.

\section{Finite length wires}

In the $\mathrm{SC}^{\star}$ phase at zero temperature, the only low energy effects of dissipation in our nanowire model arise from the global $k=0$ mode and thus depend solely on the total resistance of the wire. In finite wires this would mean that at very low temperatures, when the wire acts like a single Josephson junction, its total resistance will determine whether it is superconducting or resistive. At higher temperatures $T>c / L$, the behavior of the wire could exhibit a KT crossover which is tuned by the stiffness, or thickness, of the wire. 
We note, however, that in finite wires the resistivity may determine other parameters that are important for the superconductor-to-normal transition, such as the fugacity of the QPS ${ }^{24}$ and the strength of quantum fluctuations. ${ }^{96}$ So the superconductor-to-normal transition in finite wires is determined by both the resistance and the resistivity. A more detailed discussion will be given elsewhere. ${ }^{71}$ We should note that other effects may also be important for the case of superconducting nanowires, such as the suppressed $T_{c}$ due to Coulomb interactions as in Refs. 110 and 111.

The picture arising from our analysis conforms with and enhances that in Ref. 97. There it is assumed that a finite nanowire is shunted externally by a resistor. This resistor gives rise to a zero-temperature SC-NOR transition, but the plasmon degrees of freedom in the wire give rise to sharp crossovers. Our picture puts the picture of Büchler et al. ${ }^{97}$ on firm microscopic footing by showing that, quite generically, the resistivity of the wire needs to be taken into account as a single resistor that shunts the entire length of the wire.

\section{Experiments on nanowires}

The hypothesis that dissipation plays an important role in superconducting nanowires is supported by the recent experiments of Bezryadin et al.,23,24,89 in which the superconductor-to-normal transition was observed to depend on the normal state resistance of the wire. Later experiments ${ }^{24}$ on longer wires showed a transition that depended on the resistivity of the wire, rather than its total resistance. Bollinger et al. ${ }^{90}$ recently carried an extensive study of shorter wires $(\sim 200 \mathrm{~nm})$, and observed results consistent with both previous experiments. At this point it is still not clear whether the zero-temperature SC-metal transition in finite-length amorphous nanowires depends on their total resistance, or primarily on the resistivity.

\section{E. Charge discreteness effects}

Before turning to broader implications, we briefly raise various issues. The most serious caveat to our results arise from aspects of charge discreteness that we have ignored. In the formalism we use, the charge discreteness not only gives the basic periodicity of the superconducting phase $\phi$ of the Josephson coupling, but also implies periodic boundary conditions modulo $2 \pi$ in the imaginary time path integral on $(0, \beta)$, e.g., in Eq. (12). We have ignored the effects of nontrivial windings of the phase and thus the linear $\frac{d \phi}{d \tau}$ term that only matters when $\phi(\tau+\beta) \neq \phi(\tau)$. These can cause Berryphase-like interference effects among QPS that we have ignored.

For single Josephson junctions, an approach to dissipation that respects charge quantization is to explicitly analyze quasiparticle tunneling as carried out, for example, in Ref. 37. It is found that the quasiparticle tunneling has very similar effect to Ohmic dissipation when the (continuum) electrostatic equilibrium corresponds to an integer number of Cooper pairs on each electrode. The generalization of this to JJ chains in the absence of dissipation has been analyzed in Ref. 112 and others: ${ }^{113}$ the simple model we use is only directly applicable when there is an integer number of Cooper pairs per grain (or other commensurate filling for which the grains can act in groups). At incommensurate filling factors the Cooper pairs are always delocalized in the absence of randomness. Hence, naively, one would expect that if the Ohmic shunt resistors are replaced by quasiparticle tunneling and motion of discrete electrons, and the conversion resistance by quasiparticle creation and annihilation processes caused by the dynamics of the superconducting phase $\phi$ the conclusions of our paper would only be valid for integer number of Cooper pairs per grain. This additional effect of charge discreteness, which would severely restrict the applicability of our results, is most likely to be problematic when the shunt and conversion resistances are high: this will increase phase fluctuations and result, in any case, in charging effects destroying the superconductivity as we have found. When the resistances are low-the regime in which the FSC phase is predicted-it is less clear what the effects of charge discreteness are as the number of Cooper pairs on a grain is no longer a good quantum number. We leave for future work this issue, as well as the question of whether near to, but on the normal side of transitions, there is an intermediate-or fuller-temperature range over which our results apply.

The case of effectively commensurate filling, corresponding to the coefficient of the $\partial / \partial \tau, \widetilde{n}_{S}=0$ is the case in which a transition is possible even in the absence of dissipation. ${ }^{112}$ From the superconducting side, this should be driven by proliferation of individual QPS as the absence of resistive interactions, dipoles have finite action, and thus will always occur as fluctuations.

\section{F. Randomness}

For another issue, the effects of randomness, the above discussion is also relevant. Giamarchi and Schulz analyzed a strictly one-dimensional boson system with a random potential. ${ }^{60}$ They discussed the important role of the random Berry's phase, which controls the equilibrium local density of bosons, and found a transition between the superfluid and the insulating Bose glass phases that is driven by pinning of the density fluctuations of the superconducting phase by the random potential. How wires with spatially random properties would behave in the presence of "normal" carriers and dissipation is unclear. A similar question arises for the KTlike transition predicted by Altman et al. ${ }^{113}$ for a commensurately filled chain of grain with random self capacitance and nearest neighbor Josephson interactions.

In the strong randomness limit of a JJ chain with dissipation, the superconducting transition is effectively local as we have found here, but with subtle interactions between the local phase slips. With strong randomness, the transition should thus be dominated by weak links on many length and energy scales, and may be controlled by an infinite randomness quantum critical point as found in other random quantum systems. ${ }^{114-117}$

The effects of randomness on normal carriers need also be considered. Localization effects in a nanowire typically become important below the Thouless temperature which is roughly the spacing of energy levels in a segment of length 
such that its total resistance is of order $R_{Q}$. Simple estimates of this for a JJ chain suggest this temperature is of the same order as $T^{*}$ up to factors of $R / R_{Q}$ - which make the Thouless temperature much lower when this factor is small, i.e., strong dissipation. But with a system like that of Fig. 13, the tunneling barriers between the grains and the normal nanowire both play roles: in this situation, the conversion resistance, $r$ will be large, and the Thouless temperature is likely to be well below $T^{*}$. Near transitions to superconductivity, the superconducting fluctuations will affect the normal electron transport and any possible localization. As these decrease the overall resistance, it is likely that they also lower the Thouless temperature-perhaps all the way to zero at the transition. This argument is supported by the results of Giamarchi and Schulz: the $\mathrm{SC}^{\star}$ phase is stable to randomness if its stiffness parameter $K$ is sufficiently large. These, and other issues, surely merit exploring: the interplay between superconductivity and randomness is still largely an open field.

\section{G. Higher dimensions}

Finally, we briefly discuss the implications of our results for thin films and bulk materials. The percolation picture of the superconducting to normal transition in two and three dimensional systems of grains, ${ }^{10,39}$ suggests that close to the transition the conductivity will be determined primarily by quasi-one-dimensional percolation paths. This, and the local effects of dissipation on each junction, suggest that many of the effects that we study here for one-dimensional systems should apply to transitions in granular systems more generally. These effects may lead to various interesting crossovers, including the possibility of non-monotonic temperature dependence of the resistivity (see also Ref. 59) and quasimetallic behavior near to putative superconducting-insulator transitions as observed in a variety of systems..$^{14,118,119}$ One of the most intriguing (and poorly understood) features of such transitions in thin films is the existence of a "supermetallic phase" characterized by a small but apparently weakly temperature resistivity down to very low temperaturesextrapolating, it appears, to zero temperature. ${ }^{63}$ This behavior is in striking contrast with the theoretical picture of the superconductor to insulator transition in two dimensional systems presented in Refs. 84, 112, and 120-123. In this, the resistivity in the limit of low temperatures should go to either zero or infinity. The origin of the observed metallic behavior in thin films is still unclear, although several groups have recently addressed this problem. ${ }^{16,62,124-126}$ A possible origin of such a "supermetallic phase" in thin films are the percolation aspects of the transition combined with effects analogous to those that we have discussed in this paper. In particular, these should be significant when, near the transition, there are many low energy excitations that give rise to dissipation.

Note added. Recently, Ref. 127, which analyzes a similar problem with similar methods, appeared online.

\section{ACKNOWLEDGMENTS}

It is a pleasure to thank A. Bezryadin, M.P.A. Fisher,
J. Free, B. I. Halperin, A. Kapitulnik, S. Kivelson, W. Neils, M. Tinkham, S. Sachdev, D. Shahar, and G. Zarand for useful discussions. This work has been supported in part by the NSF via Grants No. DMR-0229243 (D.S.F.) and No. DMR0132874 (E.D.), and by the Israel-U.S. BSF and an Alona grant (Y.O.).

\section{APPENDIX: DERIVATION OF LOW ENERGY ACTION AND JUSTIFICATION OF APPROXIMATIONS}

In this appendix we outline derivations of various lowenergy forms of the action that are used in the text, from the basic action (12) of the chain in terms of the superconducting phase and the electrochemical potential of each grain:

$$
\begin{aligned}
S_{\text {chain }}= & S_{Q}+S_{\mathrm{dis}}^{r}+S_{\mathrm{dis}}^{R}+S_{J} \\
= & \int d \tau \sum_{i} \frac{1}{\left(D_{N}+D_{S}+C D_{N} D_{S}\right)}\left\{\frac { 1 } { 2 } \left[V_{\mathrm{SC}}\left(x_{i}, \tau\right)\right.\right. \\
& \left.\left.-V_{N}\left(x_{i}, \tau\right)\right]^{2}+\frac{1}{2} C D_{S} V_{N}\left(x_{i}, \tau\right)^{2}+\frac{1}{2} C D_{N} V_{\mathrm{SC}}\left(x_{i}, \tau\right)^{2}\right\} \\
& +\beta \sum_{\omega_{n}}\left[\frac{1}{\left|\omega_{n}\right| r}\left|V_{N}\left(\omega_{n}\right)-V_{\mathrm{SC}}\left(\omega_{n}\right)\right|^{2}\right] \frac{1}{\hbar} \\
& +\beta \sum_{\omega_{n}} \int \frac{d k}{2 \pi}\left[\frac{1}{\left|\omega_{n}\right| R}\left|k V_{N}\left(k, \omega_{n}\right) a\right|^{2}\right] \frac{1}{\hbar} \\
& -\int_{0}^{\beta} d \tau \sum_{i}\left\{E_{J} \cos \left[\phi_{i+1}(\tau)-\phi_{i}(\tau)\right]\right\}
\end{aligned}
$$

at zero temperature, the sums over Matsubara frequencies can be replaced by integrals $\beta \Sigma_{\omega_{n}} \rightarrow \int \frac{d \omega}{2 \pi}$ which we will use herein.

From Eq. (A1), one can integrate out the normal voltages $V_{N}(x, \tau)$ to obtain

$$
\begin{aligned}
S_{\text {chain }}= & S_{J}+\frac{1}{2} \int \frac{d \omega}{2 \pi} \int \frac{d k}{2 \pi}\left\{[\widetilde{C}-F(k, \omega)]\left|V_{\mathrm{SC}}(k, \omega)\right|^{2}\right. \\
& \left.+\frac{2-2 \cos (k a)}{a R|\omega|}\left|V_{\mathrm{SC}}(k, \omega)\right|^{2}\right\},
\end{aligned}
$$

where

$$
\left.F=\frac{\left(B+\frac{a^{2} k^{2}}{R|\omega|}\right)^{2}}{\left(C_{N S}+\frac{\frac{1}{r}+\frac{a^{2} k^{2}}{R}}{|\omega|}\right.}\right)
$$

with

$$
\tilde{C}=\frac{C\left(D_{N}+D_{S}\right)}{D_{N}+D_{S}+C D_{N} D_{S}} \approx C,
$$

$$
C_{N S}=\frac{1+D_{S} C}{D_{N}+D_{S}+C D_{N} D_{S}},
$$




$$
B=-\frac{D_{S} C}{D_{N}+D_{S}+C D_{N} D_{S}} .
$$

In expression (A2), the last term and the $\widetilde{C}$ term (which is a slightly modified capacitance in the limit $\left.C D_{N, S} \ll 1\right)$ comprise the usual action for a dissipatively shunted JJ. Together with these two terms, the third, complicated looking, term, with coefficient $F(k, \omega)$, gives rise to the two fluid behavior. It is from the frequency dependence of $F$ that the basic energy scale

$$
T^{*}=\frac{\hbar}{C_{N S}}\left(\frac{1}{r}+\frac{1}{R}\right)
$$

arises. The low energy regime of interest is $\omega \ll T^{*}$. Note that because of the local nature of some of the important physics, we must take the low frequency limit at fixed $k$; if the limit is taken in the opposite order, the results can be very misleading.

At low energies, the action becomes

$$
\begin{aligned}
S_{\text {chain }}= & S_{J}+\frac{1}{2} \int \frac{d \omega}{2 \pi} \int \frac{d k}{2 \pi}\left(\widetilde{C}\left|V_{\mathrm{SC}}(k, \omega)\right|^{2}\right. \\
& \left.+\frac{a}{|\omega|} \frac{2-2 \cos (k a)}{R+r[2-2 \cos (k a)]}\left|V_{\mathrm{SC}}(k, \omega)\right|^{2}\right),
\end{aligned}
$$

which, on approximating $\widetilde{C}$ by $C$, is the form of the action we use in both the strong and weak coupling limits. In the weak coupling limit, we drop the capacitative term as this is unimportant at low frequencies.

In the strong coupling limit, the capacitative term is important and the Villain approximation for the Josephson coupling and ensuing transformations can be carried out straightforwardly as discussed in Sec. III A. In general, this is only appropriate for low energies, in particular for $\omega \ll \Omega_{L R}$, the inductive relaxation rate

$$
\Omega_{L R}=\left(\frac{2 e}{\hbar}\right)^{2} E_{J} R,
$$

since $E_{J}$ is inversely proportional to the "kinetic inductance" $L_{J}=\left(\frac{\hbar}{2 e}\right)^{2} \frac{1}{E_{J}}$ of the junction. Note that $\Omega_{L R}$ is the same order as the plasma frequency of the junctions, reduced from this by a factor of $K R / R_{Q}$ which is of order unity in most of the regimes of interest. Thus the Villain approximation is valid wherever we have used it.

The action in terms of phase slips, the interactions between them, and the equivalent sine-Gordon representation in terms of the dual fields $\theta$ and $\psi$ can be derived straightforwardly. Note that we could have gone more directly to the sine-Gordon representation by decoupling the intergrain terms in Eq. (A1) with the fields $\psi$ and $\theta$, then integrating out $V_{N}$ and $\phi$. The periodicity of $\phi$ then gives rise to an integer constraint on $(\theta+\psi) / 2 \pi$ which after integrating out the high energy fluctuations, becomes the $\cos (\theta+\psi)$ in the sineGordon action. The appropriate low frequency approximations appear naturally in the intermediate representation. In particular, the quadratic coupling between $\theta$ and $\psi$ that appears if this route is followed, is unimportant at low frequencies.
${ }^{1}$ C. P. Slichter, Principles of Magnetic Resonance (SpringerVerlag, Berlin, 1978).

${ }^{2}$ A. Abragam, The Principles of Nuclear Magnetism (Oxford University Press, London, 1961).

${ }^{3}$ C. W. Gardiner and P. Zoller, Quantum Noise (Springer, New York, 2000).

${ }^{4}$ Y. Imry, Introduction to Mesoscopic Physics (Oxford University Press, Oxford, 1997).

${ }^{5}$ D. J. Van Harlingen, T. L. Robertson, B. L. T. Plourde, P. A. Reichardt, T. A. Crane, and J. Clarke, Phys. Rev. B 70, 064517 (2004).

${ }^{6}$ M. A. Nielsen and I. L. Chuang, Quantum Computing and Quantum Information (Cambridge University Press, Cambridge, UK, 2000).

${ }^{7}$ J. A. Hertz, Phys. Rev. B 14, 1165 (1976).

${ }^{8}$ A. J. Millis, Phys. Rev. B 48, 7183 (1993).

${ }^{9}$ S. Sachdev, Quantum Phase Transitions (Cambridge University Press, Cambridge, 1999).

${ }^{10}$ E. Shimshoni, A. Auerbach, and A. Kapitulnik, Phys. Rev. Lett. 80, 3352 (1998).

${ }^{11}$ S. Chakravarty, G. L. Ingold, S. Kivelson, and A. Luther, Phys. Rev. Lett. 56, 2303 (1986)

${ }^{12}$ K.-H. Wagenblast, A. van Otterlo, G. Schön, and G. T. Zimányi,
Phys. Rev. Lett. 78, 1779 (1997).

${ }^{13}$ A. Larkin, Ann. Phys. (N.Y.) 8, 785 (1999).

${ }^{14}$ A. Kapitulnik, N. Mason, S. A. Kivelson, and S. Chakravarty, cond-mat/0009201 (unpublished).

${ }^{15}$ G. Sambandamurthy, L. W. Engel, A. Johansson, and D. Shahar, Phys. Rev. Lett. 92, 107005 (2004).

${ }^{16}$ D. Dalidovich and P. Phillips, Phys. Rev. Lett. 84, 737 (2000).

${ }^{17}$ R. K. Lindell, J. Delahaye, M. A. Sillanpää, T. T. Heikkilä, E. B. Sonin, and P. J. Hakonen, Phys. Rev. Lett. 93, 197002 (2004).

${ }^{18}$ W. Lu, K. D. Maranowski, and A. J. Rimberg, Phys. Rev. B 65, 060501(R) (2002)

${ }^{19}$ M. Watanabe and D. B. Haviland, Phys. Rev. B 67, 094505 (2003).

${ }^{20}$ D. Pekker, A. Bezryadin, D. S. Hopkins, and P. M. Goldbart, Phys. Rev. B 72, 104517 (2005).

${ }^{21}$ S. Sachdev, P. Werner, and M. Troyer, Phys. Rev. Lett. 92, 237003 (2004).

${ }^{22}$ M. Tinkham, J. U. Free, C. N. Lau, and N. Markovic, Phys. Rev. B 68, 134515 (2003).

${ }^{23}$ A. Bezryadin, C. N. Lau, and M. Tinkham, Nature (London) 404, 971 (2000).

${ }^{24}$ C. N. Lau, N. Markovic, M. Bockrath, A. Bezryadin, and M. Tinkham, Phys. Rev. Lett. 87, 217003 (2001). 
${ }^{25}$ A. O. Caldeira and A. J. Leggett, Phys. Rev. Lett. 46, 211 (1981).

${ }^{26}$ A. O. Caldeira and A. J. Leggett, Ann. Phys. (N.Y.) 149, 374 (1983)

${ }^{27}$ A. J. Leggett, S. Chakravarty, A. T. Dorsey, M. P. A. Fisher, A. Garg, and W. Zwerger, Rev. Mod. Phys. 59, 1 (1987).

${ }^{28}$ S. Chakravarty, Phys. Rev. Lett. 49, 681 (1982).

${ }^{29}$ A. Schmid, Phys. Rev. Lett. 51, 1506 (1983).

${ }^{30}$ S. A. Bulgadaev, JETP Lett. 39, 315 (1984).

${ }^{31}$ S. E. Korshunov, Sov. Phys. JETP 66, 872 (1987).

${ }^{32}$ E. Simanek, Inhomogeneous Superconductivity (Oxford University Press, New York, 1994).

${ }^{33}$ U. Weiss, Quantum Dissipative Systems (World Scientific, Singapore, 2001).

${ }^{34}$ G. L. Ingold and Y. V. Nazarov, in Single Charge Tunneling, edited by H. Grabert and M. H. Devoret, NATO ASI Series B: Physics Vol. 294 (Plenum Press, New York, 1992), pp. 21-107.

${ }^{35}$ K. Andersson and D. B. Haviland, Phys. Rev. B 67, 092507 (2003).

${ }^{36}$ J. S. Penttilä, Ü. Parts, P. J. Hakonen, M. A. Paalanen, and E. B. Sonin, Phys. Rev. Lett. 82, 1004 (1999).

${ }^{37}$ G. Schön and A. D. Zaikin, Phys. Rep. 198, 238 (1990).

${ }^{38}$ S. V. Panyukov and A. D. Zaikin, Phys. Lett. A 156, 119 (1991).

${ }^{39}$ M. P. A. Fisher, Phys. Rev. B 36, 1917 (1987).

${ }^{40}$ S. Chakravarty, G. L. Ingold, S. Kivelson, and G. Zimányi, Phys. Rev. B 37, 3283 (1988).

${ }^{41}$ P. A. Bobbert, R. Fazio, G. Schön, and A. D. Zaikin, Phys. Rev. B 45, 2294 (1992).

${ }^{42}$ S. E. Korshunov, Sov. Phys. JETP 68, 609 (1989).

${ }^{43}$ S. E. Korshunov, Europhys. Lett. 9, 107 (1989).

${ }^{44}$ W. Zwerger, Europhys. Lett. 9, 421 (1989).

${ }^{45}$ P. A. Bobbert, R. Fazio, G. Schön, and G. T. Zimányi, Phys. Rev. B 41, 4009 (1990).

${ }^{46}$ R. Fazio and H. van der Zant, Phys. Rep. 355, 235 (2001).

${ }^{47}$ S. Tewari, J. Toner, and S. Chakravarty, Phys. Rev. B 73, 064503 (2006).

${ }^{48}$ C. D. Chen, D. Delsing, and D. B. H. T. Claeson, Phys. Scr. T42, 182 (1992).

${ }^{49}$ E. Chow, P. Delsing, and D. B. Haviland, Phys. Rev. Lett. 81, 204 (1998).

${ }^{50}$ E. Chow, P. Delsing, and D. B. Haviland, Phys. Rev. Lett. 81, 204 (1998).

${ }^{51}$ D. B. Haviland, E. Chow, and P. Delsing, Physica B 284, 1808 (2000).

${ }^{52}$ D. B. Haviland, K. Anderson, and P. Agren, Low Temp. Phys. 118, 733 (2000).

${ }^{53}$ A. J. Rimberg, T. R. Ho, C. Kurdak, J. Clarke, K. L. Campman, and A. C. Gossard, Phys. Rev. Lett. 78, 2632 (1997).

${ }^{54}$ Y. Takahide, R. Yagi, A. Kanda, Y. Ootuka, and S. I. Kobayashi, Phys. Rev. Lett. 85, 1974 (2000).

${ }^{55}$ C. D. Chen, D. Delsing, D. B. Haviland, and T. Claeson, Phys. Scr., T T42, 182 (1992).

${ }^{56} \mathrm{M}$. Watanabe and D. B. Haviland, in Studies of High Temperature Superconductors (Advances in Research and Applications), BSCCO Tapes and More on Josephson Structures and Superconducting Electronics, edited by A. Narlikar (Nova Science Publishers, New York, 2002, ISBN 1-590-33343-8), Vol. 43, Chap. 6.

${ }^{57}$ R. S. Newrock, C. J. Lobb, U. Geigenmuller, and M. Octavio, Solid State Phys. 54, 263 (2000).

${ }^{58}$ H. Miyazaki, Y. Takahide, A. Kanda, and Y. Ootuka, Phys. Rev.
Lett. 89, 197001 (2002).

${ }^{59}$ G. Refael, E. Demler, Y. Oreg, and D. S. Fisher, Phys. Rev. B 68, 214515 (2003)

${ }^{60}$ T. Giamarchi and H. J. Schulz, Phys. Rev. B 37, 325 (1988).

${ }^{61}$ K.-H. Wagenblast, A. van Otterlo, G. Schön, and G. T. Zimányi, Phys. Rev. Lett. 79, 2730 (1997).

${ }^{62}$ A. Vishwanath, J. E. Moore, and T. Senthil, Phys. Rev. B 69, 054507 (2004).

${ }^{63}$ N. Mason and A. Kapitulnik, Phys. Rev. B 65, 220505(R) (2002).

${ }^{64}$ J. Clarke, U. Eckern, A. Schmid, G. Schon, and M. Tinkham, Phys. Rev. B 20, 3933 (1979).

${ }^{65}$ A. D. Zaikin, D. S. Golubev, A. van Otterlo, and G. T. Zimányi, Phys. Rev. Lett. 78, 1552 (1997).

${ }^{66}$ J. M. Duan, Phys. Rev. Lett. 74, 5128 (1995).

${ }^{67}$ N. Giordano and E. R. Schuler, Phys. Rev. Lett. 63, 2417 (1989).

${ }^{68}$ N. Giordano, Phys. Rev. Lett. 61, 2137 (1988).

${ }^{69}$ N. Giordano Phys. Rev. B 41, 6350 (1990).

${ }^{70}$ N. Giordano and E. R. Schuler, Physica B 203, 460 (1994).

${ }^{71}$ G. Refael, E. Demler, Y. Oreg, and D. S. Fisher (unpublished).

${ }^{72}$ W. J. Skocpol, M. R. Beasley, and M. Tinkham, J. Low Temp. Phys. 16, 145 (1974).

${ }^{73}$ M. Tinkham, Rev. Mod. Phys. 46, 587 (1974).

${ }^{74}$ B. Ivlev and N. Kopnin, Adv. Phys. 33, 47 (1984).

${ }^{75}$ We can speculate, however, that the superfluid-normal relaxation time may arise either from Andreev processes at a normalsuperconducting interface in a non-uniform grain, or due to random pair-breaking impurities. See also the discussion in Ref. 59.

${ }^{76}$ M. Büttiker, H. Thomas, and A. Pretre, Phys. Lett. A 364-369, 364 (1993).

${ }^{77}$ R. M. Bradley and S. Doniach, Phys. Rev. B 30, 1138 (1984).

${ }^{78}$ V. Gurarie and A. M. Tsvelik, J. Low Temp. Phys. 135, 245 (2004).

${ }^{79}$ A. Kamenev and A. Andreev, Phys. Rev. B 60, 2218 (1999).

${ }^{80}$ J. E. Mooij and G. Schön, Phys. Rev. Lett. 55, 114 (1985).

${ }^{81}$ B. Camarota, F. Parage, F. Balestro, P. Delsing, and O. Buisson, Phys. Rev. Lett. 86, 480 (2001).

${ }^{82}$ G. Refael, Ph.D. thesis, Harvard University, Cambridge, MA, 2003.

${ }^{83}$ Neutrality for a given time slice of the phase slip gas is not required. Single phase slips may be triggered by charging interaction between nearest neighbors. Such effects are irrelevant in the low energy effective field theory, and therefore are not explicitly included in the ensuing analysis. An important point is that a phase slip is an instanton that shifts a phase difference between neighboring grains by $2 \pi$. This kink is then propagated to the rest of the chain.

${ }^{84}$ K. B. Efetov, Sov. Phys. JETP 51, 5 (1980).

${ }^{85}$ A. M. van den Brink, A. A. Odintsov, P. A. Bobbert, and G. Schön, Z. Phys. B: Condens. Matter 85, 459 (1991).

${ }^{86}$ P. Werner, G. Refael, and M. Troyer, J. Stat. Mech.: Theory Exp. 2005, P12003.

${ }^{87}$ P. Minnhagen, Rev. Mod. Phys. 59, 1001 (1987).

${ }^{88}$ M. P. A. Fisher and W. Zwerger, Phys. Rev. B 32, 6190 (1985).

${ }^{89}$ M. Tinkham and C. N. Lau, Appl. Phys. Lett. 80, 2946 (2002).

${ }^{90}$ A. T. Bollinger, A. Rogachev, M. Remeika, and A. Bezryadin, Phys. Rev. B 69, 180503(R) (2004).

${ }^{91}$ A. Bollinger, A. Rogachev, and A. Bezryadin, Europhys. Lett. 76, 505 (2006).

${ }^{92}$ J. S. Langer and V. Ambegaokar, Phys. Rev. 164, 498 (1967).

${ }^{93}$ D. E. McCumber and B. I. Halperin, Phys. Rev. B 1, 1054 
(1970).

${ }^{94}$ S. Saito and Y. Murayama, Phys. Lett. A 139, 85 (1989).

${ }^{95}$ Y. Chang, Phys. Rev. B 54, 9436 (1996).

${ }^{96}$ D. S. Golubev and A. D. Zaikin, Phys. Rev. B 64, 014504 (2001).

${ }^{97}$ H. P. Büchler, V. B. Geshkenbein, and G. Blatter, Phys. Rev. Lett. 92, 067007 (2004).

${ }^{98}$ A. van Otterlo, M. Feigel'man, V. Geshkenbein, and G. Blatter, Phys. Rev. Lett. 75, 3736 (1995).

${ }^{99}$ E. B. Sonin, V. B. Geshkenbein, A. van Otterlo, and G. Blatter, Phys. Rev. B 57, 575 (1998).

${ }^{100}$ P. Ao and D. J. Thouless, Phys. Rev. Lett. 70, 2158 (1993).

${ }^{101}$ M. P. A. Fisher and D. H. Lee, Phys. Rev. B 39, 2756 (1989).

${ }^{102}$ D. R. Hamann, Phys. Rev. Lett. 26, 1030 (1971).

${ }^{103}$ P. Noziéres and C. T. de Dominicis, Phys. Rev. 178, 1097 (1969).

${ }^{104}$ K. K. Likharev and A. B. Zorin, J. Low Temp. Phys. 59, 347 (1985).

${ }^{105}$ D. C. Ralph, S. Gueron, C. T. Black, and M. Tinkham, Physica B 280, 420 (2000).

${ }^{106}$ For the clean $N$ wire and perfect $S-N$ contact the SC-normal transition may arise due to a different physical mechanism, in which superconducting condensation energy on the grains competes with the energy cost of inducing pairing correlations in the $N$ wire, see, e.g., Refs. 107 and 108.

${ }^{107}$ B. Spivak, A. Zyuzin, and M. Hruska, Phys. Rev. B 64, 132502 (2001).

${ }^{108}$ M. V. Feigelman and A. I. Larkin, cond-mat/9803006 (unpublished).

${ }^{109}$ S. J. Putterman, Superfluid Hydrodynamics (North-Holland, Am- sterdam, 1978).

${ }^{110}$ Y. Oreg and E. Demler, in Electronic Correlations: From Mesoto Nano-physics, Proceedings of the XXXVI Rencontres de Moriond, edited by G. M. T. Martin and J. T. T. Van (2001).

${ }^{111}$ Y. Oreg and A. M. Finkel'stein, Phys. Rev. Lett. 84, 191 (2000).

${ }^{112}$ M. P. A. Fisher, P. B. Weichman, G. Grinstein, and D. S. Fisher, Phys. Rev. B 40, 546 (1989).

${ }^{113}$ E. Altman, Y. Kafri, A. Polkovnikov, and G. Refael, Phys. Rev. Lett. 93, 150402 (2004).

${ }^{114}$ D. S. Fisher, Phys. Rev. B 50, 3799 (1994).

${ }^{115}$ D. S. Fisher, Phys. Rev. B 51, 6411 (1995).

${ }^{116}$ C. A. Doty and D. S. Fisher, Phys. Rev. B 45, 2167 (1992).

${ }^{117}$ R. N. Bhatt and P. A. Lee, Phys. Rev. Lett. 48, 344 (1982).

${ }^{118}$ A. Yazdani and A. Kapitulnik, Phys. Rev. Lett. 74, 3037 (1995).

${ }^{119}$ A. Kapitulnik, N. Mason, S. A. Kivelson, and S. Chakravarty, Phys. Rev. B 63, 125322 (2001).

${ }^{120}$ S. Doniach, Phys. Rev. B 24, 5063 (1981).

${ }^{121}$ M.-C. Cha, M. P. A. Fisher, S. M. Girvin, M. Wallin, and A. P. Young, Phys. Rev. B 44, 6883 (1991).

${ }^{122}$ A. van Otterlo, K.-H. Wagenblast, R. Fazio, and G. Schön, Phys. Rev. B 48, 3316 (1993).

${ }^{123}$ T. K. Kopec and J. V. Jose, Phys. Rev. B 60, 7473 (1999).

${ }^{124}$ D. Dalidovich and P. Phillips, Phys. Rev. B 64, 184511 (2001).

${ }^{125}$ D. Das and S. Doniach, Phys. Rev. B 60, 1261 (1999).

${ }^{126}$ V. M. Galitski, G. Refael, M. P. A. Fisher, and T. Senthil, Phys. Rev. Lett. 95, 077002 (2005).

${ }^{127}$ P. Goswami and S. Chakravarty, Phys. Rev. B 73, 094516 (2006). 